Supporting Information for:

\title{
Thermodynamic Understanding of an Aza-Michael Reaction Enables 5-Step Synthesis of the Potent Integrin Inhibitor MK-0429
}

Anya Gupta, Matthew L. Condakes*

Department of Small Molecule Drug Discovery, Bristol Myers Squibb Company, 100 Binney St. Cambridge, MA 02142

*matthew.condakes@bms.com

\section{Table of Contents:}

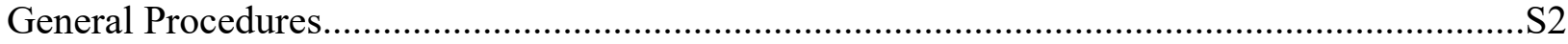

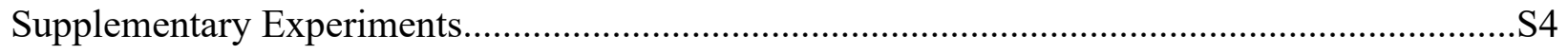

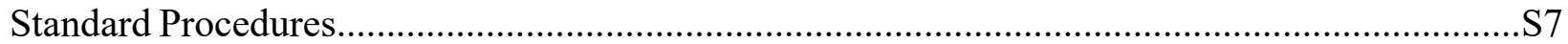

Compound Preparation and Characterization Data...............................................................S8

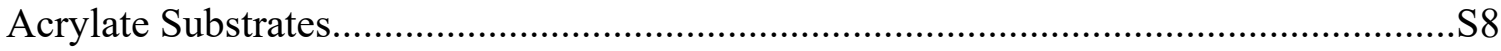

Products Exploring Acrylate Scope..................................................................... 12

Products Exploring Nucleophile Scope...............................................................S2 1

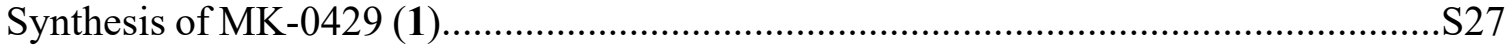

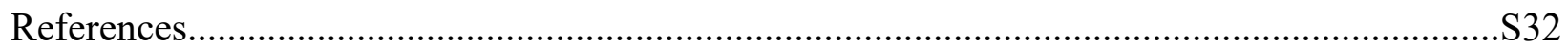

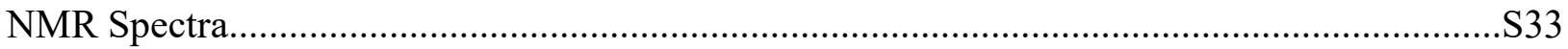




\section{General Procedures:}

All reactions were performed in Chemglass reaction vials affixed with pressure release caps under ambient atmosphere, unless otherwise indicated. Reactions that required heating were carried out in temperature-controlled heating blocks, unless otherwise indicated. Air- and moisture-sensitive liquids were transferred via syringe. Volatile solvents were removed under reduced pressure rotary evaporation below $35^{\circ} \mathrm{C}$. Reaction progress was monitored by LCMS (Shimadzu Nexera X2) or by analytical thin-layer chromatography (TLC). TLC was performed using glass plates pre-coated with silica gel ( $250 \mu \mathrm{m}$ thickness, $10 \mu \mathrm{m}$ particle size, Millipore Sigma) impregnated with a fluorescent indicator $(254 \mathrm{~nm})$ and plates were visualized by exposure to ultraviolet light (UV). Automated column chromatography was performed on CombiFlash ${ }^{\circledR}$ NextGen 300+ machines using pre-packed columns of silica gel (RediSep ${ }^{\circledR}$ Normal-phase Silica Flash Columns).

Anhydrous acetonitrile (MeCN), dichloromethane (DCM), dimethylformamide (DMF), dioxane, and tetrahydrofuran (THF) were purchased from Millipore Sigma and used as received. Pyrazole (4) and tert-butyl cinnamate (5) were purchased from Millipore Sigma and were used as received. Acrylate precursors, acrylates, and heterocyclic nucleophiles were purchased from commercial suppliers and used as received. Tert-butyl 7-chloro-3,4-dihydro-1,8-naphthyridine-1(2H)carboxylate (13) is commercially available but was also prepared according to the literature protocol. ${ }^{1}$ SPhos Pd G1, MTBE adduct was purchased from Millipore Sigma and used as received. All other solvents and reagents were purchased at the highest commercial grade available and used as received, without additional purification.

Proton nuclear magnetic resonance $\left({ }^{1} \mathrm{H} \mathrm{NMR}\right)$ spectra and carbon nuclear magnetic resonance $\left({ }^{13} \mathrm{C}\right.$ NMR) spectra were recorded on Bruker Avance $500(500 \mathrm{MHz} / 126 \mathrm{MHz})$ or Bruker Avance NEO $600(600 \mathrm{MHz} / 151 \mathrm{MHz})$ spectrometers at $23{ }^{\circ} \mathrm{C}$. Fluorine nuclear magnetic resonance $\left({ }^{19} \mathrm{~F}\right.$ NMR) spectra were recorded on a Bruker Avance $500(471 \mathrm{MHz})$ spectrometer at 23 ${ }^{\circ} \mathrm{C}$. Proton chemical shifts are expressed as parts per million (ppm, $\delta$ scale) and are referenced to residual protium in the NMR solvent $\left(\mathrm{CHCl}_{3}: \delta 7.26, \mathrm{CD}_{2} \mathrm{HOD}: \delta 3.31\right)$. Carbon chemical shifts are expressed as parts per million ( $\mathrm{ppm}, \delta$ scale) and are referenced to the carbon resonance of the NMR solvent $\left(\mathrm{CDCl}_{3}: \delta 77.16, \mathrm{CD}_{3} \mathrm{OD}: \delta 49.00\right)$. Fluorine chemical shifts are expressed as parts per million (ppm, $\delta$ scale) and are not additionally referenced. Data are represented as follow: chemical shift, multiplicity $(\mathrm{s}=$ singlet, $\mathrm{d}=$ doublet, $\mathrm{t}=$ triplet, $\mathrm{q}=$ quartet, $\mathrm{m}=$ multiplet, $\mathrm{br}=$ broad), coupling constant $(J)$ in Hertz $(\mathrm{Hz})$, and integration. High resolution mass spectrometry 
(HRMS) analyses were performed on a Fourier Transform Orbitrap mass spectrometer (QExactive+, Thermo Fisher Scientific, San Jose, CA) in positive or negative ionization electrospray mode operating at 35,000 resolution (full width at half height maximum, FWHM). The instrument was daily calibrated according to manufacturer's specifications resulting in mass accuracy of or better than $5 \mathrm{ppm}$. The operating software Xcalibur was used to process the obtained data. 


\section{Supplementary Experiment 1. Racemization of Enantiopure 6}

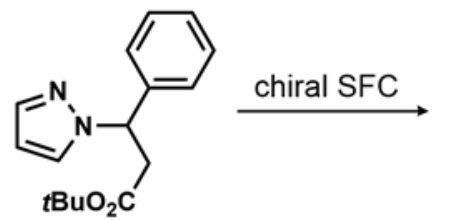

$( \pm)-6$

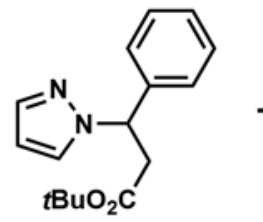

$6 a$ (first eluting)<smiles>CC(OC(C)(C)C)C(c1ccccc1)n1cccn1</smiles>

$6 \mathrm{~b}$ (second eluting)

Racemic 6 (80 mg) was separated into two enantiomers by chiral supercritical fluid chromatography (SFC) (Phenomenex Amylose-1, $i \mathrm{PrOH} / \mathrm{CO}_{2} / \mathrm{NH}_{4} \mathrm{OH}=15 / 85 / 0.001$, flow rate $=100$ $\mathrm{mL} / \mathrm{min}, \lambda=260 \mathrm{~nm}) \mathrm{tR}=2.0 \mathrm{~min}(\mathbf{6 a}), 2.5 \min (\mathbf{6 b})$. The absolute steroechemistry of each enantiomer was not determined. 6a (31 mg, 39\%) was the first eluting isomer and $6 \mathbf{b}$ ( $29 \mathrm{mg}$, $36 \%$ ) was the second eluting isomer. The ee of each isomer was determined by analytical SFC (Chiralpak AD-H, $i \mathrm{PrOH} / \mathrm{CO}_{2} / \mathrm{NH}_{4} \mathrm{OH}=10 / 90 / 0.001$, flow rate $=2.0 \mathrm{~mL} / \mathrm{min}$ ) $\mathrm{tR}=1.2 \mathrm{~min}$ (6a), $3.4 \mathrm{~min}(\mathbf{6 b})$ and was found to be $>99 \%$ for both $\mathbf{6 a}$ and $\mathbf{6 b}$.

Racemic 6, prior to chiral separation:

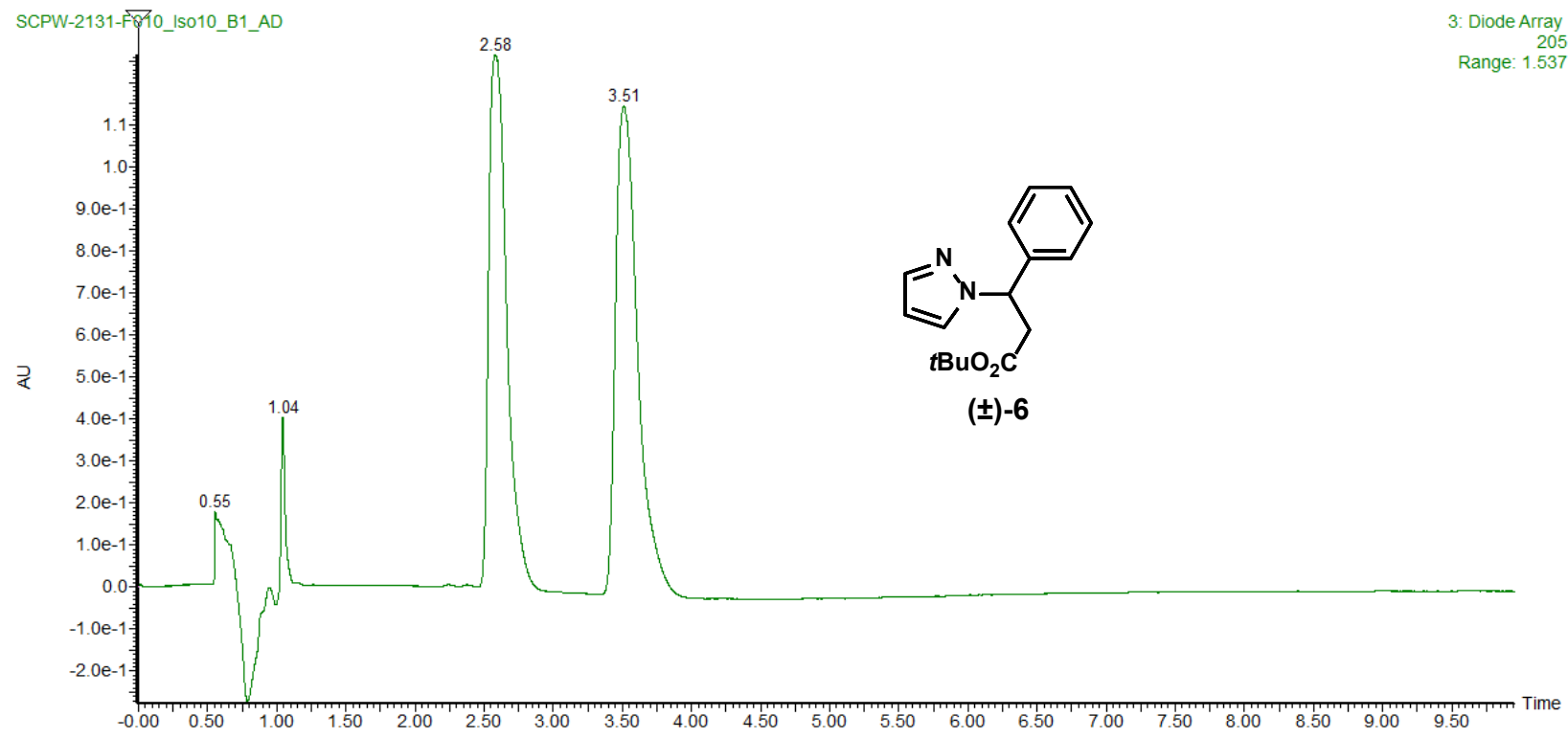


Separated enantiomers $\mathbf{6 a}$ and $\mathbf{6 b}$ :

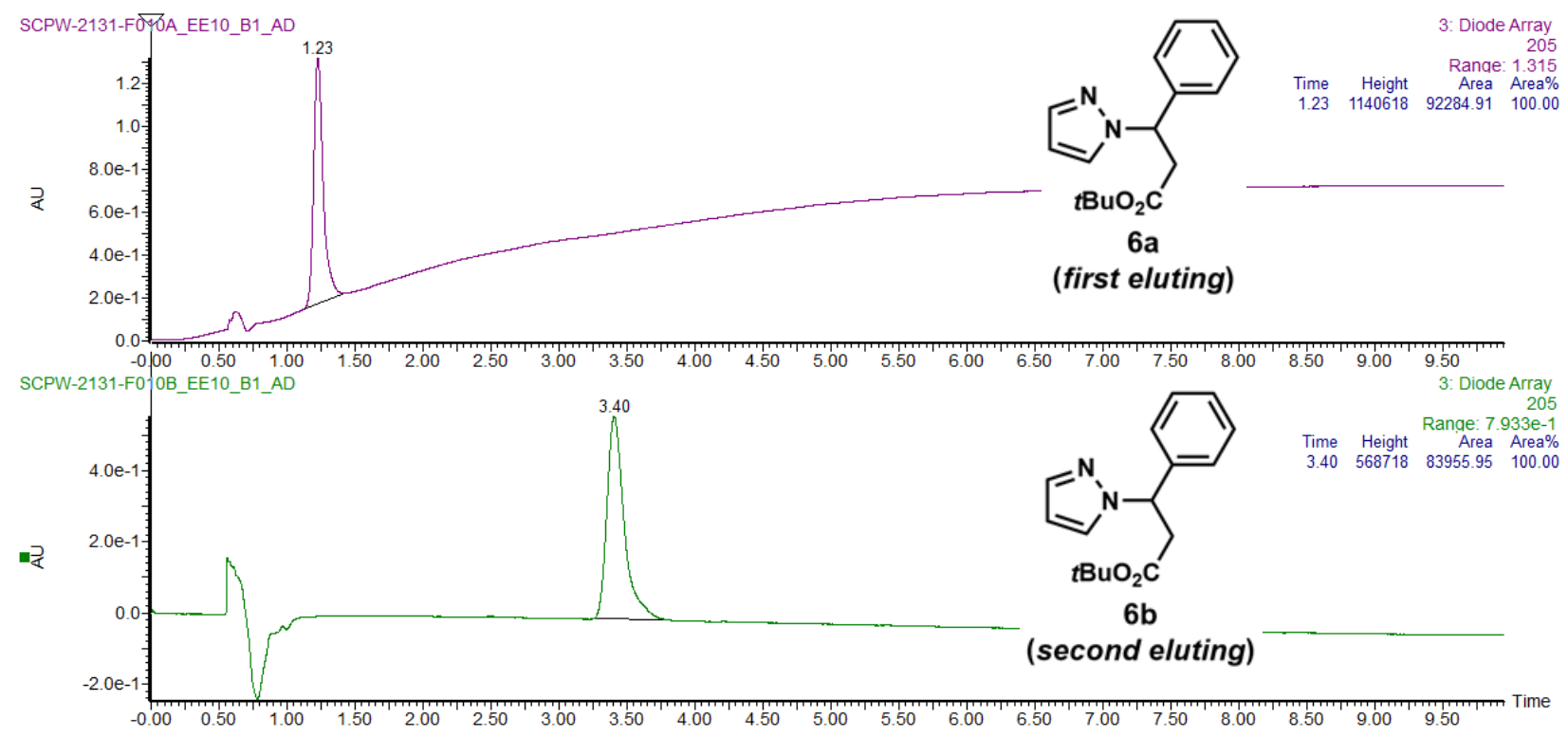

Each separated enantiomer was then subjected to the following modified reaction conditions:

Pyrazole (1.4 mg, $0.02 \mathrm{mmol}, 0.2$ equiv) was dissolved in $\mathrm{MeCN}(300 \mu \mathrm{L})$ and potassium tertbutoxide ( $2.2 \mathrm{mg}, 0.02 \mathrm{mmol}, 0.2$ equiv) was added. The solution was stirred at room temperature for $5 \mathrm{~min}$. Then, $\mathbf{6 a}(31 \mathrm{mg}, 0.1 \mathrm{mmol}, 1.0$ equiv) or $\mathbf{6 b}(29 \mathrm{mg}, 0.1 \mathrm{mmol}, 1.0$ equiv) was added and the reaction mixture was heated at $80{ }^{\circ} \mathrm{C}$ for $1 \mathrm{~h}$. An aliquot $(5 \mu \mathrm{L})$ was taken from each reaction mixture and was analyzed by chiral SFC using the preparative method. The ee of 6a was determined to be $4.5 \%$ and the $e e$ of $\mathbf{6} \mathbf{b}$ was determined to be $-0.3 \%$.

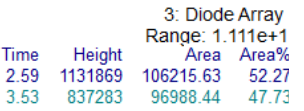

6a following reaction conditions:

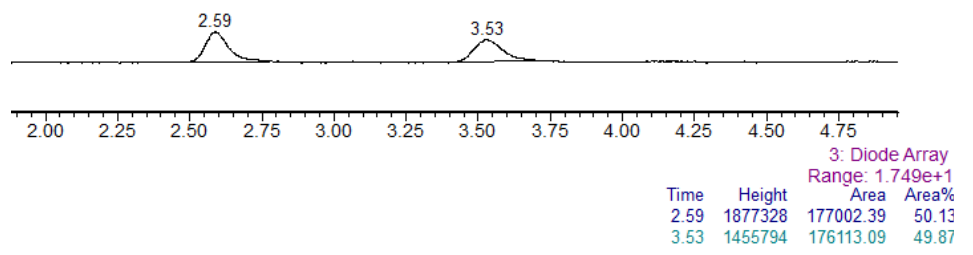

6b following reaction conditions:

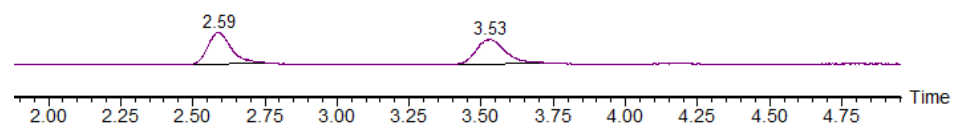




\section{Supplementary Experiment 2. Temperature Cycling of Michael Addition}

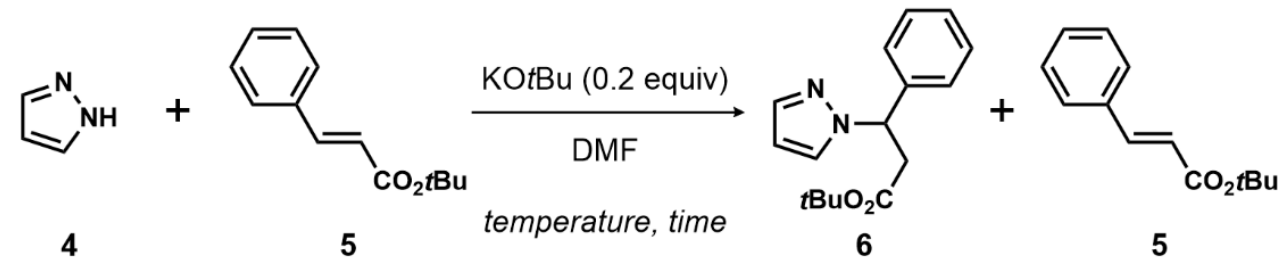

Pyrazole (68 mg, $1.0 \mathrm{mmol}, 1.0$ equiv) and tert-butyl cinnamate (204 mg, $1.0 \mathrm{mmol}, 1.0$ equiv) were dissolved in DMF (0.5 mL) and potassium tert-butoxide (22 mg, $0.2 \mathrm{mmol}, 0.2$ equiv) was added. The reaction mixture was heated at $100{ }^{\circ} \mathrm{C}$ for $30 \mathrm{~min}$. An aliquot $(<5 \mu \mathrm{L})$ was taken from this hot solution and analyzed by UPLC/MS (Acquity BEH C18, $\mathrm{MeCN} / \mathrm{H}_{2} \mathrm{O} / \mathrm{TFA}=$ $5 / 95 / 0.05 \rightarrow 95 / 5 / 0.05$, flow rate $=1.0 \mathrm{~mL} / \mathrm{min}, \lambda=220 \mathrm{~nm}) \mathrm{tR}=1.27 \mathrm{~min}(\mathbf{6}), 1.44 \mathrm{~min}(\mathbf{5})$. The ratio of 6:5 was observed to be 1:1.7 (N.B.: 5 absorbs UV radiation at $220 \mathrm{~nm}$ more strongly than 6). The reaction mixture was then cooled to $60^{\circ} \mathrm{C}$ and was stirred at that temperature for $1.5 \mathrm{~h}$. Once again, an aliquot was taken from this hot solution to be analyzed by LC/MS. The ratio of 6:5 was observed to now be $1: 1$. The reaction mixture was heated to $100{ }^{\circ} \mathrm{C}$ and was stirred at that temperature for $30 \mathrm{~min}$. A similar LC/MS analysis was performed and the ratio of 6:5 was observed to have returned to $1: 1.7$. This cycling was performed an additional two times, with no erosion of the observed ratios. Finally, the reaction mixture was allowed to cool to room temperature and was stirred for $42 \mathrm{~h}$. LC/MS analysis indicated the ratio of 6:5 was now 2.5:1. A tabular summary of these observations can be found below, with corresponding excerpted chromatograms:

\begin{tabular}{r|cccccc} 
Time $(\mathrm{h})$ & 0.5 & 2 & 2.5 & 4 & 4.5 & 48 \\
$\mathrm{~T}\left({ }^{\circ} \mathrm{C}\right)$ & 100 & 60 & 100 & 60 & 100 & 23 \\
$\mathbf{6 : 5}$ & $1: 1.7$ & $1: 1$ & $1: 1.8$ & $1.1: 1$ & $1: 1.7$ & $2.5: 1$
\end{tabular}
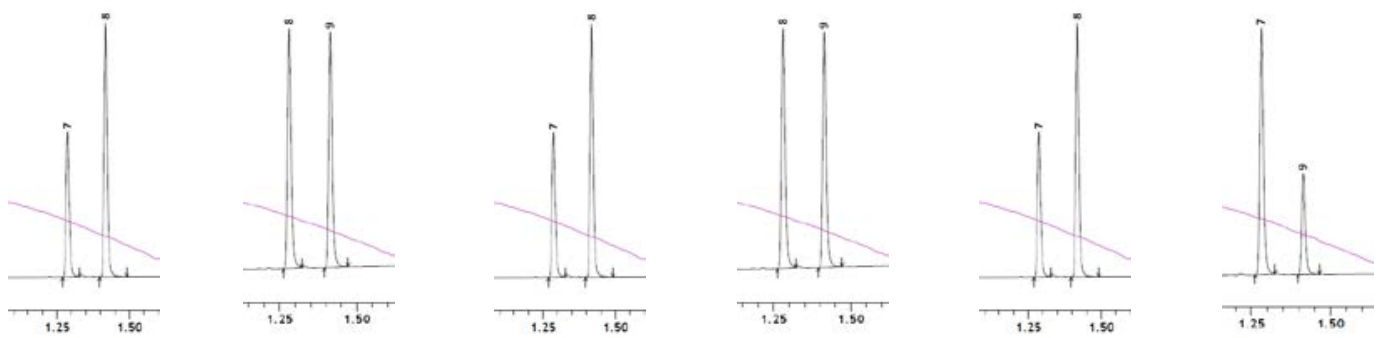


\section{Experimental Section:}

Standard Procedure for the Synthesis of Acrylate Substrates.

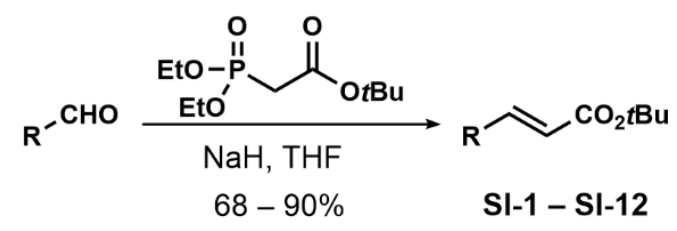

To solution of tert-butyl diethylphosphonoacetate $(9.0 \mathrm{mmol}, 3.0$ equiv) in THF $(15 \mathrm{~mL})$ at room temperature was added sodium hydride (60 wt\% dispersion in mineral oil, unwashed, $9.0 \mathrm{mmol}$, 3.0 equiv) portionwise. Once bubbling had subsided, aldehyde (3.0 mmol, 1.0 equiv) was added and the reaction mixture was stirred until product formation was complete (typically $<1 \mathrm{~h}$ ). The reaction mixture was diluted with $\mathrm{Et}_{2} \mathrm{O}(45 \mathrm{~mL})$ and was quenched with a saturated aqueous ammonium chloride solution $(15 \mathrm{~mL})$. The layers were separated, and the organic phase was further washed with brine $(15 \mathrm{~mL})$, dried over magnesium sulfate, filtered, and concentrated. The crude residue was purified by flash chromatography $(0 \rightarrow 10 \%$ EtOAc/hexanes for aromatic and aliphatic substrates, and $10 \rightarrow 25 \%$ EtOAc/hexanes for heteroaromatic substrates) to afford the desired products as solid, single isomers $(E: Z>20: 1)$, except when otherwise indicated.

\section{Standard Procedure for the Aza-Michael Addition.}

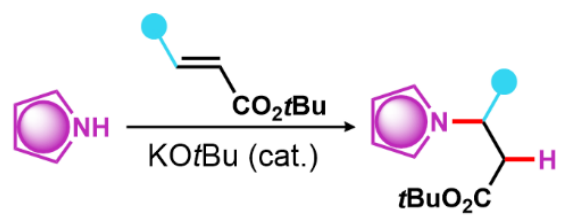

Pyrazole (1.0 mmol, 1.0 equiv) was dissolved in $\mathrm{MeCN}(0.5 \mathrm{~mL})$ and $\mathrm{KO} t \mathrm{Bu}(0.2 \mathrm{mmol}, 0.2$ equiv) was added followed by acrylate $(1.0 \mathrm{mmol}, 1.0$ equiv). The resulting solution was heated in a reaction vial on a heating block at $80{ }^{\circ} \mathrm{C}$ for $30 \mathrm{~min}, 60^{\circ} \mathrm{C}$ for $1.5 \mathrm{~h}$, and was then stirred at room temperature overnight $(>12 \mathrm{~h})$. The reaction mixture was directly purified by column chromatography $(10 \rightarrow 50 \%$ EtOAc/hexanes for all substrates $)$ to afford the desired Michael adducts. 


\section{Acrylate Starting Materials:}

$\overbrace{\mathrm{CO}_{2} \mathrm{tBu}}$

Acrylate SI-1: The standard procedure was followed with 2-methylbenzaldehyde $(400 \mathrm{mg}, 3.3 \mathrm{mmol})$ to afford, after column chromatography $(0 \rightarrow 10 \%$ EtOAc/hexanes), SI-1 (630 mg, $2.9 \mathrm{mmol}, 87 \%$ ) as a white solid. Characterization data were in agreement with the literature. ${ }^{2}$

${ }^{1} \mathrm{H}$ NMR (500 MHz, $\left.\mathrm{CDCl}_{3}\right) \delta 7.89$ (d, $\left.J=15.9 \mathrm{~Hz}, 1 \mathrm{H}\right), 7.54$ (dd, J=8.1, $\left.1.3 \mathrm{~Hz}, 1 \mathrm{H}\right), 7.26$ (td, $J=7.4,1.1 \mathrm{~Hz}, 1 \mathrm{H}), 7.20$ (dd, $J=8.1,7.4 \mathrm{~Hz}, 1 \mathrm{H}), 7.19$ (d, $J=7.4 \mathrm{~Hz}, 1 \mathrm{H}), 6.30$ (d, $J=15.9 \mathrm{~Hz}, 1 \mathrm{H})$, $2.43(\mathrm{~s}, 3 \mathrm{H}), 1.54(\mathrm{~s}, 9 \mathrm{H}) ;{ }^{13} \mathrm{C}\left\{{ }^{1} \mathrm{H}\right\} \mathrm{NMR}\left(126 \mathrm{MHz}, \mathrm{CDCl}_{3}\right) \delta 166.6,141.4,137.6,133.7,130.8$, $129.8,126.5,126.4,121.3,80.6,28.4(3 \mathrm{C}), 19.9$.

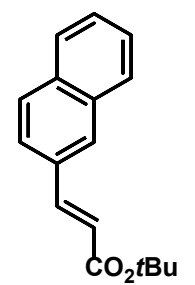

Acrylate SI-2: The standard procedure was followed with 2-naphthaldehyde (500 mg, $3.2 \mathrm{mmol})$ to afford, after column chromatography ( $0 \rightarrow 10 \%$ EtOAc/hexanes), SI-2 (565 mg, $2.2 \mathrm{mmol}, 69 \%$ ) as a white solid. Characterization data were in agreement with the literature. ${ }^{3}$

${ }^{1} \mathrm{H}$ NMR $\left(600 \mathrm{MHz}, \mathrm{CDCl}_{3}\right) \delta 7.91(\mathrm{~s}, 1 \mathrm{H}), 7.87$ - $7.80(\mathrm{~m}, 2 \mathrm{H}), 7.82$ (d, J=8.5 Hz, 1H), $7.75(\mathrm{~d}, J=15.9 \mathrm{~Hz}, 1 \mathrm{H}), 7.66(\mathrm{dd}, J=8.5,1.5 \mathrm{~Hz}, 1 \mathrm{H}), 7.53-7.48(\mathrm{~m}, 2 \mathrm{H}), 6.49$ (d, $J=15.9$ $\mathrm{Hz}, 1 \mathrm{H}), 1.56(\mathrm{~s}, 9 \mathrm{H}) ;{ }^{13} \mathrm{C}\left\{{ }^{1} \mathrm{H}\right\} \mathrm{NMR}\left(151 \mathrm{MHz}, \mathrm{CDCl}_{3}\right) \delta 166.5,143.7,134.2,133.5,132.3$, $129.7,128.7,128.6,127.9,127.2,126.8,123.7,120.5,80.7,28.4$ (3C).

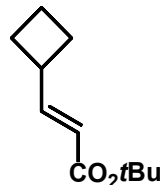

Acrylate SI-3: The standard procedure was followed with cyclobutanecarbaldehyde $(168 \mathrm{mg}, 2.0 \mathrm{mmol})$ to afford, after column chromatography $(0 \rightarrow 10 \%$ EtOAc/hex${ }^{1} \mathrm{H}$ NMR $\left(600 \mathrm{MHz}, \mathrm{CDCl}_{3}\right) \delta 6.93(\mathrm{dd}, J=15.5,6.8 \mathrm{~Hz}, 1 \mathrm{H}), 5.65(\mathrm{~d}, J=15.5 \mathrm{~Hz}, 1 \mathrm{H}), 3.10$ - 3.01 $(\mathrm{m}, 1 \mathrm{H}), 2.19-2.11(\mathrm{~m}, 2 \mathrm{H}), 1.97-1.90(\mathrm{~m}, 3 \mathrm{H}), 1.87-1.80(\mathrm{~m}, 1 \mathrm{H}), 1.47(\mathrm{~s}, 9 \mathrm{H}) ;{ }^{13} \mathrm{C}\left\{{ }^{1} \mathrm{H}\right\} \mathrm{NMR}$ $\left(151 \mathrm{MHz}, \mathrm{CDCl}_{3}\right) \delta 166.6,151.5,120.9,80.1,37.7,28.3$ (3C), 28.0 (2C), 18.7; HRMS (ESI) m/z: $[\mathrm{M}+\mathrm{H}]^{+}$calcd for $\mathrm{C}_{11} \mathrm{H}_{19} \mathrm{O}_{2}$ 183.1385; found 183.1380 .

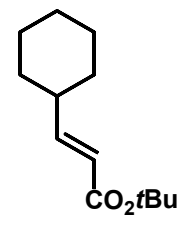

Acrylate SI-4: The standard procedure was followed with cyclohexanecarbaldehyde (224 mg, $2.0 \mathrm{mmol}$ ) to afford, after column chromatography $(0 \rightarrow 10 \%$ 
EtOAc/hexanes), SI-4 (380 mg, $1.8 \mathrm{mmol}, 90 \%$ ) as a colorless oil. Characterization data were in agreement with the literature. ${ }^{4}$

${ }^{1} \mathrm{H}$ NMR $\left(500 \mathrm{MHz}, \mathrm{CDCl}_{3}\right) \delta 6.79(\mathrm{dd}, J=15.7,6.7 \mathrm{~Hz}, 1 \mathrm{H}), 5.66(\mathrm{dd}, J=15.7,1.4 \mathrm{~Hz}, 1 \mathrm{H}), 2.13$ - $2.04(\mathrm{~m}, 1 \mathrm{H}), 1.77$ - $1.71(\mathrm{~m}, 4 \mathrm{H}), 1.68$ - 1.62 (m, 1H), 1.46 (s, 9H), 1.30 - 1.22 (m, 2H), 1.20 $1.07(\mathrm{~m}, 3 \mathrm{H}) ;{ }^{13} \mathrm{C}\left\{{ }^{1} \mathrm{H}\right\} \mathrm{NMR}\left(126 \mathrm{MHz}, \mathrm{CDCl}_{3}\right) \delta 166.7,153.2,120.7,80.0,40.4,31.9$ (2C), $28.3(3 \mathrm{C}), 26.1,25.9(2 \mathrm{C})$.

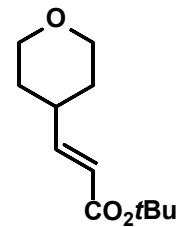

Acrylate SI-5: The standard procedure was followed with tetrahydro-2H-pyran-4carbaldehyde (450 mg, $3.94 \mathrm{mmol})$ to afford, after column chromatography $(0 \rightarrow$ 10\% EtOAc/hexanes), SI-5 (702 mg, $3.3 \mathrm{mmol}, 84 \%$ ) as a colorless oil.

${ }^{1} \mathrm{H}$ NMR $\left(600 \mathrm{MHz}, \mathrm{CDCl}_{3}\right) \delta 6.79(\mathrm{dd}, J=15.7,6.4 \mathrm{~Hz}, 1 \mathrm{H}), 5.72$ (d, $J=15.7 \mathrm{~Hz}$, $1 \mathrm{H}), 3.98$ (dd, $J=11.8,4.2 \mathrm{~Hz}, 2 \mathrm{H}), 3.43$ (t, $J=11.6 \mathrm{~Hz}, 2 \mathrm{H}), 2.39-2.31$ (m, 1H), 1.69 - 1.63 (m, 2H), $1.56-1.50(\mathrm{~m}, 2 \mathrm{H}), 1.48(\mathrm{~s}, 9 \mathrm{H}) ;{ }^{13} \mathrm{C}\left\{{ }^{1} \mathrm{H}\right\} \mathrm{NMR}\left(151 \mathrm{MHz}, \mathrm{CDCl}_{3}\right) \delta 166.3,150.8,121.7$, 80.5, $67.6(2 \mathrm{C}), 37.6,31.6(2 \mathrm{C}), 28.3$ (3C). HRMS (ESI) m/z: $[\mathrm{M}+\mathrm{H}]^{+}$calcd for $\mathrm{C}_{12} \mathrm{H}_{21} \mathrm{O}_{3}$ 213.1491; found 213.1483.

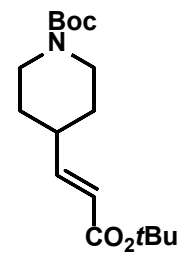

Acrylate SI-6: The standard procedure was followed with tert-butyl-4formylpiperidine-1-carboxylate $(500 \mathrm{mg}, 2.3 \mathrm{mmol})$ to afford, after column chromatography $(0 \rightarrow 10 \%$ EtOAc/hexanes), SI-6 (560 mg, $1.8 \mathrm{mmol}, 77 \%)$ as a thick, ${ }^{1} \mathrm{H}$ NMR $\left(500 \mathrm{MHz} \mathrm{CDCl}_{3}\right) \delta 6.77$ (dd, $\left.J=15.8,6.6 \mathrm{~Hz}, 1 \mathrm{H}\right), 5.71$ (dd, $\left.J=15.8,1.4 \mathrm{~Hz}, 1 \mathrm{H}\right), 4.09$ (br s, 2H), $2.81-2.67(\mathrm{~m}, 2 \mathrm{H}), 2.30-2.20(\mathrm{~m}, 1 \mathrm{H}), 1.74-1.66(\mathrm{~m}, 2 \mathrm{H}), 1.47(\mathrm{~s}, 9 \mathrm{H}), 1.44(\mathrm{~s}$, 9H), 1.37 - $1.27(\mathrm{~m}, 2 \mathrm{H}) ;{ }^{13} \mathrm{C}\left\{{ }^{1} \mathrm{H}\right\}$ NMR $\left(126 \mathrm{MHz}, \mathrm{CDCl}_{3}\right) \delta 166.2,154.9,150.6,121.9,80.4$, 79.6, 43.5 (br s, 2C), 38.6, 30.8 (br s, 2C), 28.6 (s, 3C), 28.3 (s, 3C); HRMS (ESI) m/z: [M+H] calcd for $\mathrm{C}_{17} \mathrm{H}_{30} \mathrm{NO}_{4} 312.2175$, found 312.2166.

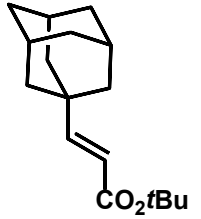

Acrylate SI-7: The standard procedure was followed with adamantane-1carbaldehdye (385 mg, $2.3 \mathrm{mmol})$ to afford, after column chromatography $(0 \rightarrow 10 \%$ EtOAc/hexanes), SI-7 (420 mg, $1.6 \mathrm{mmol}, 68 \%$ ) as a white solid.

${ }^{1} \mathrm{H}$ NMR $\left(500 \mathrm{MHz}, \mathrm{CDCl}_{3}\right) \delta 6.71(\mathrm{~d}, J=16.0 \mathrm{~Hz}, 1 \mathrm{H}), 5.58(\mathrm{~d}, J=16.0 \mathrm{~Hz}, 1 \mathrm{H})$, 2.00 (br s, 3H), 1.74 (br d, $J=12.3 \mathrm{~Hz}, 3 \mathrm{H}), 1.65$ (br d, $J=12.3 \mathrm{~Hz}, 3 \mathrm{H}), 1.62$ (d, $J=2.4 \mathrm{~Hz}, 6 \mathrm{H})$, 
$1.48(\mathrm{~s}, 9 \mathrm{H}) ;{ }^{13} \mathrm{C}\left\{{ }^{1} \mathrm{H}\right\} \mathrm{NMR}\left(126 \mathrm{MHz}, \mathrm{CDCl}_{3}\right) \delta 167.1,157.8,118.3,80.1,41.3(3 \mathrm{C}), 36.8(3 \mathrm{C})$, 35.7, 28.33 (3C), 28.26 (3C); HRMS (ESI) m/z: $[\mathrm{M}+\mathrm{H}]^{+}$calcd for $\mathrm{C}_{17} \mathrm{H}_{27} \mathrm{O}_{2}$ 263.2011, found 263.2012 .

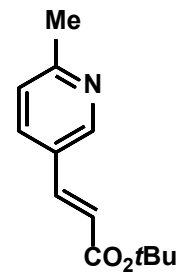

Acrylate SI-8: The standard procedure was followed with 6-methylnicotinaldehyde $(1.0 \mathrm{~g}, 8.3 \mathrm{mmol})$ to afford, after column chromatography $(10 \rightarrow 25 \%$ EtOAc/hexanes), SI-8 (1.6 g, $7.3 \mathrm{mmol}, 88 \%)$ as an off-white solid.

7.53 (d, $J=16.0 \mathrm{~Hz}, 1 \mathrm{H}), 7.16$ (d, $J=8.1 \mathrm{~Hz}, 1 \mathrm{H}), 6.37$ (d, $J=16.0 \mathrm{~Hz}, 1 \mathrm{H}), 2.57$ (s, 3H), 1.52 (s, $9 \mathrm{H}) ;{ }^{13} \mathrm{C}\left\{{ }^{1} \mathrm{H}\right\} \mathrm{NMR}\left(126 \mathrm{MHz}, \mathrm{CDCl}_{3}\right) \delta 165.9,160.2,149.2,140.0,134.5,127.8,123.5,121.4$, 81.0, 28.3 (3C), 24.6; HRMS (ESI) m/z: [M+H] calcd for $\mathrm{C}_{13} \mathrm{H}_{18} \mathrm{NO}_{2} 220.1338$, found 220.1348 .

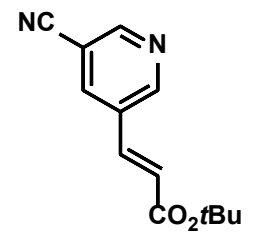

Acrylate SI-9: The standard procedure was followed with 5-formylnicotinonitrile $(1.0 \mathrm{~g}, 7.6 \mathrm{mmol})$ to afford, after column chromatography $(10 \rightarrow 25 \%$ EtOAc/hexanes), SI-9 (1.4 g, $6.1 \mathrm{mmol}, 80 \%)$ as an off-white solid.

${ }^{1} \mathrm{H}$ NMR $\left(600 \mathrm{MHz}, \mathrm{CDCl}_{3}\right) \delta 8.90(\mathrm{~d}, J=1.8 \mathrm{~Hz}, 1 \mathrm{H}), 8.83(\mathrm{~d}, J=1.8 \mathrm{~Hz}, 1 \mathrm{H})$, $8.06(\mathrm{t}, J=1.8 \mathrm{~Hz}, 1 \mathrm{H}), 7.54(\mathrm{~d}, J=16.0 \mathrm{~Hz}, 1 \mathrm{H}), 6.50(\mathrm{~d}, J=16.0 \mathrm{~Hz}, 1 \mathrm{H}), 1.54(\mathrm{~s}, 9 \mathrm{H}) ;{ }^{13} \mathrm{C}\left\{{ }^{1} \mathrm{H}\right\}$ NMR (151 MHz, $\left.\mathrm{CDCl}_{3}\right) \delta 164.8,152.6,152.3,137.2$ (2C, overlapping"), 131.0, 125.4, 116.1, 110.6, 81.8, 28.2 (3C), ${ }^{*}$ overlapping peaks confirmed by HSQC; HRMS (ESI) m/z: $[\mathrm{M}+\mathrm{H}]^{+}$calcd for $\mathrm{C}_{13} \mathrm{H}_{15} \mathrm{~N}_{2} \mathrm{O}_{2} 231.1134$, found 231.1120 .

Acrylate SI-10: The standard procedure was followed with 2-methylpyrimidine-5-
carbaldehyde (611 mg, $5.0 \mathrm{mmol})$ to afford, after column chromatography $(10 \rightarrow$
$25 \%$ EtOAc/hexanes), SI-10 $(860 \mathrm{mg}, 3.9 \mathrm{mmol}, 78 \%)$ as a white solid.
$\mathrm{CO}_{2} \mathrm{BBu}^{1}{ }^{1} \mathrm{H} \mathrm{NMR}\left(600 \mathrm{MHz}, \mathrm{CDCl}_{3}\right) \delta 8.75(\mathrm{~s}, 2 \mathrm{H}), 7.48(\mathrm{~d}, J=16.2 \mathrm{~Hz}, 1 \mathrm{H}), 6.46(\mathrm{~d}, J=16.2$ $\mathrm{Hz}, 1 \mathrm{H}), 2.76(\mathrm{~s}, 3 \mathrm{H}), 1.53$ (s, 9H); ${ }^{13} \mathrm{C}\left\{{ }^{1} \mathrm{H}\right\} \mathrm{NMR}\left(151 \mathrm{MHz}, \mathrm{CDCl}_{3}\right) \delta 169.1,165.3,155.8(2 \mathrm{C})$, 136.4, 125.5, 123.2, 81.4, 28.3 (3C), 26.1; HRMS (ESI) m/z: $[\mathrm{M}+\mathrm{H}]^{+}$calcd for $\mathrm{C}_{12} \mathrm{H}_{17} \mathrm{~N}_{2} \mathrm{O}_{3}$ 237.1239 , found 237.1232 . 
OMe Acrylate SI-11: The standard procedure was followed with 2-methoxypyrimidine-5carbaldehyde $(2.0 \mathrm{~g}, 14.5 \mathrm{mmol})$ to afford, after column chromatography $(10 \rightarrow 25 \%$ EtOAc/hexanes), SI-11 (2.8 g, $11.9 \mathrm{mmol}, 82 \%)$ as a white solid.

$\mathrm{CO}_{2} \mathrm{tBu}{ }^{1} \mathrm{H}$ NMR $\left(600 \mathrm{MHz}, \mathrm{CDCl}_{3}\right) \delta 8.64(\mathrm{~s}, 2 \mathrm{H}), 7.46(\mathrm{~d}, J=16.2 \mathrm{~Hz}, 1 \mathrm{H}), 6.37$ (d, $J=16.2$ $\mathrm{Hz}, 1 \mathrm{H}), 4.04$ (s, 3H), 1.53 (s, 9H); ${ }^{13} \mathrm{C}\left\{{ }^{1} \mathrm{H}\right\} \mathrm{NMR}\left(151 \mathrm{MHz}, \mathrm{CDCl}_{3}\right) \delta 166.1,165.6,158.5$ (2C), 136.3, 122.6, 121.2, 81.2, 55.5, 28.3 (3C); HRMS (ESI) m/z: calcd for $\mathrm{C}_{12} \mathrm{H}_{17} \mathrm{~N}_{2} \mathrm{O}_{3} 237.1239$, found 237.1228 .

$\mathrm{CO}_{2} \mathrm{Bu}_{\mathrm{Bu}}$

Acrylate SI-12: The standard procedure was followed with quinoline-6-carbaldehyde (450 mg, $2.9 \mathrm{mmol})$ to afford, after column chromatography $(10 \rightarrow 25 \%$ EtOAc/hexanes), SI-12 (635 mg, $2.5 \mathrm{mmol}, 87 \%$ ) as a white solid. Characterization data were in argreement with the literature. ${ }^{5}$

${ }^{1} \mathrm{H}$ NMR $\left(500 \mathrm{MHz}, \mathrm{CDCl}_{3}\right) \delta 8.92(\mathrm{dd}, J=4.3,1.7 \mathrm{~Hz}, 1 \mathrm{H}), 8.16(\mathrm{~d}, J=8.3 \mathrm{~Hz}, 1 \mathrm{H})$, 8.08 (d, $J=9.4 \mathrm{~Hz}, 1 \mathrm{H}), 7.88$ (s, 1H), 7.89 (dd, $J=9.4,1.7 \mathrm{~Hz}, 1 \mathrm{H}), 7.74$ (d, $J=16.0 \mathrm{~Hz}, 1 \mathrm{H}), 7.42$ $(\mathrm{dd}, J=8.2,4.3 \mathrm{~Hz}, 1 \mathrm{H}), 6.51(\mathrm{~d}, J=16.0 \mathrm{~Hz}, 1 \mathrm{H}), 1.56(\mathrm{~s}, 9 \mathrm{H}) ;{ }^{13} \mathrm{C}\left\{{ }^{1} \mathrm{H}\right\} \mathrm{NMR}\left(126 \mathrm{MHz}, \mathrm{CDCl}_{3}\right)$ $\delta 166.2,151.3,149.1,142.7,136.5,133.1,130.3,129.0,128.4,127.5,121.9,121.7,80.9,28.3$ (s, 3C). 


\section{Products Exploring Acrylate Scope:}

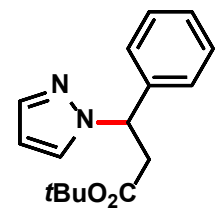

Substrate 6: The standard procedure was followed with pyrazole $(68 \mathrm{mg}, 1.0$ mmol) and tert-butyl cinnamate $(204 \mathrm{mg}, 1.0 \mathrm{mmol})$ to afford, after column chromatography $(10 \rightarrow 50 \%$ EtOAc/hexanes $), 6(223 \mathrm{mg}, 0.8 \mathrm{mmol}, 82 \%)$ as a colorless oil.

${ }^{1} \mathrm{H}$ NMR (500 MHz, $\left.\mathrm{CDCl}_{3}\right) \delta 7.54(\mathrm{dd}, J=1.8,0.5 \mathrm{~Hz}, 1 \mathrm{H}), 7.41$ (dd, $\left.J=2.4,0.5 \mathrm{~Hz}, 1 \mathrm{H}\right), 7.34$ 7.24 (m, 5H), 6.23 (dd, $J=2.4,1.8 \mathrm{~Hz}, 1 \mathrm{H}), 5.77$ (dd, $J=9.2,6.3 \mathrm{~Hz}, 1 \mathrm{H}), 3.47$ (dd, $J=15.9,9.2 \mathrm{~Hz}$, $1 \mathrm{H}), 3.05(\mathrm{dd}, J=15.9,6.3 \mathrm{~Hz}, 1 \mathrm{H}), 1.33(\mathrm{~s}, 9 \mathrm{H}) ;{ }^{13} \mathrm{C}\left\{{ }^{1} \mathrm{H}\right\} \mathrm{NMR}\left(126 \mathrm{MHz}, \mathrm{CDCl}_{3}\right) \delta 169.5,139.8$, 139.4, 129.0, 128.8 (2C), 128.2, 126.8 (2C), 105.6, 81.2, 62.2, 41.8, 27.9 (3C); UPLC (Acquity $\mathrm{BEH} \mathrm{C18,} \mathrm{MeCN} / \mathrm{H}_{2} \mathrm{O} / \mathrm{TFA}=5 / 95 / 0.05 \rightarrow 95 / 5 / 0.05$, flow rate $\left.=1.0 \mathrm{~mL} / \mathrm{min}, \lambda=220 \mathrm{~nm}\right) \mathrm{tR}=$ $1.27 \mathrm{~min}$; HRMS (ESI) m/z: [M+H] $]^{+}$calcd for $\mathrm{C}_{16} \mathrm{H}_{21} \mathrm{~N}_{2} \mathrm{O}_{2} 273.1603$; found 273.1593.

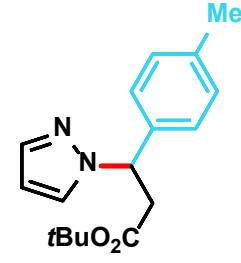

Substrate 7a: The standard procedure was followed with pyrazole $(68 \mathrm{mg}, 1.0$ mmol) and tert-butyl (E)-3-(p-tolyl)acrylate $(218 \mathrm{mg}, 1.0 \mathrm{mmol})$ to afford, after column chromatography $(10 \rightarrow 50 \%$ EtOAc/hexanes $), 7 \mathbf{a}(218 \mathrm{mg}, 0.8 \mathrm{mmol}$, $76 \%)$ as a pale yellow oil.

${ }^{1} \mathrm{H}$ NMR $\left(500 \mathrm{MHz}, \mathrm{CDCl}_{3}\right) \delta 7.53(\mathrm{~d}, J=1.2 \mathrm{~Hz}, 1 \mathrm{H}), 7.39$ (d, $\left.J=1.5 \mathrm{~Hz}, 1 \mathrm{H}\right), 7.18$ (d, J=7.9 Hz, 2H), $7.13(\mathrm{~d}, J=7.9 \mathrm{~Hz}, 2 \mathrm{H}), 6.23-6.21(\mathrm{~m}, 1 \mathrm{H}), 5.73$ (dd, $J=9.2,6.2 \mathrm{~Hz}, 1 \mathrm{H}), 3.45$ (dd, $J=15.9$, $9.2 \mathrm{~Hz}, 1 \mathrm{H}), 3.02(\mathrm{dd}, J=15.9,6.2 \mathrm{~Hz}, 1 \mathrm{H}), 2.31(\mathrm{~s}, 3 \mathrm{H}), 1.33(\mathrm{~s}, 9 \mathrm{H}) ;{ }^{13} \mathrm{C}\left\{{ }^{1} \mathrm{H}\right\} \mathrm{NMR}(126 \mathrm{MHz}$, $\left.\mathrm{CDCl}_{3}\right) \delta 169.6,139.3,138.0,136.8,129.5$ (2C), 129.0, 126.8 (2C), 105.5, 81.2, 62.0, 41.8, 28.0 (3C), 21.2; UPLC (Acquity BEH C18, $\mathrm{MeCN} / \mathrm{H}_{2} \mathrm{O} / \mathrm{TFA}=5 / 95 / 0.05 \rightarrow 95 / 5 / 0.05$, flow rate $=1.0$ $\mathrm{mL} / \mathrm{min}, \lambda=220 \mathrm{~nm}) \mathrm{tR}=1.35 \mathrm{~min}$; HRMS (ESI) $\mathrm{m} / \mathrm{z}$ : $[\mathrm{M}+\mathrm{H}]^{+}$calcd for $\mathrm{C}_{17} \mathrm{H}_{23} \mathrm{~N}_{2} \mathrm{O}_{2} 287.1760$; found 287.1759 .

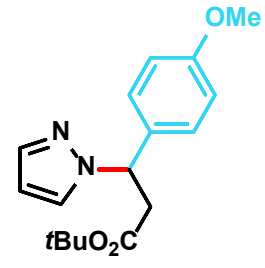

Substrate 7b: The standard procedure was followed with pyrazole $(68 \mathrm{mg}, 1.0$ $\mathrm{mmol})$ and tert-butyl (E)-3-(4-methoxyphenyl)acrylate $(234 \mathrm{mg}, 1.0 \mathrm{mmol})$ to afford, after column chromatography $(10 \rightarrow 50 \%$ EtOAc/hexanes $), 7 \mathbf{b}$ (225 mg, $0.7 \mathrm{mmol}, 74 \%$ ) as a colorless oil.

${ }^{1} \mathrm{H}$ NMR $\left(600 \mathrm{MHz}, \mathrm{CDCl}_{3}\right) \delta 7.52(\mathrm{~d}, J=1.3 \mathrm{~Hz}, 1 \mathrm{H}), 7.38$ (d, J=2.0 Hz, 1H), 7.23 (d, J=8.7 Hz, 2H), 6.85 (d, $J=8.7 \mathrm{~Hz}, 2 \mathrm{H}), 6.21$ (dd, $J=2.0,1.3 \mathrm{~Hz}, 1 \mathrm{H}), 5.71$ (dd, $J=8.9,6.6 \mathrm{~Hz}, 1 \mathrm{H}), 3.77$ (s, 
$3 \mathrm{H}), 3.44(\mathrm{dd}, J=15.8,8.9 \mathrm{~Hz}, 1 \mathrm{H}), 3.02(\mathrm{dd}, J=15.8,6.6 \mathrm{~Hz}, 1 \mathrm{H}), 1.32(\mathrm{~s}, 9 \mathrm{H}) ;{ }^{13} \mathrm{C}\left\{{ }^{1} \mathrm{H}\right\} \mathrm{NMR}$ $\left(151 \mathrm{MHz}, \mathrm{CDCl}_{3}\right) \delta 169.6,159.5,139.4,131.9,128.9,128.2$ (2C), 114.2 (2C), 105.5, 81.2, 61.8, 55.4, 41.9, 28.0 (3C); UPLC (Acquity BEH C18, $\mathrm{MeCN} / \mathrm{H}_{2} \mathrm{O} / \mathrm{TFA}=5 / 95 / 0.05 \rightarrow$ 95/5/0.05, flow rate $=1.0 \mathrm{~mL} / \mathrm{min}, \lambda=220 \mathrm{~nm}) \mathrm{tR}=1.27 \mathrm{~min} ; \mathrm{HRMS}(\mathrm{ESI}) \mathrm{m} / \mathrm{z}:[\mathrm{M}+\mathrm{H}]^{+}$calcd for $\mathrm{C}_{17} \mathrm{H}_{23} \mathrm{~N}_{2} \mathrm{O}_{3}$ 303.1709; found 303.1702.

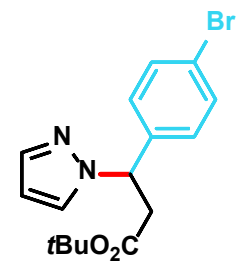

Substrate 7c: The standard procedure was followed with pyrazole $(68 \mathrm{mg}, 1.0$ $\mathrm{mmol})$ and tert-butyl (E)-3-(4-bromophenyl)acrylate $(283 \mathrm{mg}, 1.0 \mathrm{mmol})$ to afford, after column chromatography $(10 \rightarrow 50 \%$ EtOAc/hexanes), $7 \mathbf{c}(267 \mathrm{mg}, 0.8$ mmol, $76 \%)$ as an off white solid.

${ }^{1} \mathrm{H}$ NMR (600 MHz, CDCl $) \delta 7.53(\mathrm{~d}, J=1.5 \mathrm{~Hz}, 1 \mathrm{H}), 7.44(\mathrm{~d}, J=8.4 \mathrm{~Hz}, 2 \mathrm{H}), 7.41$ (d, $J=2.0 \mathrm{~Hz}$, $1 \mathrm{H}), 7.16(\mathrm{~d}, J=8.4 \mathrm{~Hz}, 2 \mathrm{H}), 6.23(\mathrm{dd}, J=2.0,1.5 \mathrm{~Hz}, 1 \mathrm{H}), 5.71$ (dd, $J=8.9,6.5 \mathrm{~Hz}, 1 \mathrm{H}), 3.43(\mathrm{dd}$, $J=16.1,8.9 \mathrm{~Hz}, 1 \mathrm{H}), 3.01(\mathrm{dd}, J=16.1,6.5 \mathrm{~Hz}, 1 \mathrm{H}), 1.33(\mathrm{~s}, 9 \mathrm{H}) ;{ }^{13} \mathrm{C}\left\{{ }^{1} \mathrm{H}\right\} \mathrm{NMR}\left(151 \mathrm{MHz}, \mathrm{CDCl}_{3}\right)$ $\delta$ 169.3, 139.7, 138.9, 132.0 (2C), 129.1, 128.6 (2C), 122.3, 105.8, 81.5, 61.6, 41.7, 28.0 (3C); UPLC (Acquity BEH C18, MeCN/ $\mathrm{H}_{2} \mathrm{O} / \mathrm{TFA}=5 / 95 / 0.05 \rightarrow 95 / 5 / 0.05$, flow rate $=1.0 \mathrm{~mL} / \mathrm{min}, \lambda$ $=220 \mathrm{~nm}$ ) $\mathrm{tR}=1.39 \mathrm{~min}$; HRMS (ESI) $\mathrm{m} / \mathrm{z}:[\mathrm{M}+\mathrm{H}]^{+}$cacld for $\mathrm{C}_{16} \mathrm{H}_{20} \mathrm{~N}_{2} \mathrm{O} 2 \mathrm{Br}$ 351.0708; found 351.0702 .

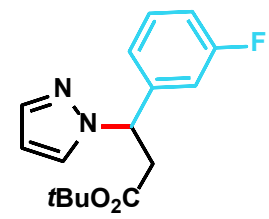

Substrate 7d: The standard procedure was followed with pyrazole (68 $\mathrm{mg}, 1.0$ $\mathrm{mmol})$ and tert-butyl (E)-3-(3-fluorophenyl)acrylate $(222 \mathrm{mg}, 1.0 \mathrm{mmol})$ to afford, after column chromatography ( $10 \rightarrow 50 \%$ EtOAc/hexanes), $7 \mathbf{d}(212 \mathrm{mg}$, $0.7 \mathrm{mmol}, 73 \%)$ as a colorless oil.

${ }^{1} \mathrm{H}$ NMR $\left(500 \mathrm{MHz}, \mathrm{CDCl}_{3}\right) \delta 7.57(\mathrm{~d}, J=1.5 \mathrm{~Hz}, 1 \mathrm{H}), 7.46$ (d, $\left.J=2.1 \mathrm{~Hz}, 1 \mathrm{H}\right), 7.31(\mathrm{td}, J=7.8,5.5$ $\mathrm{Hz}, 1 \mathrm{H}), 7.08$ (d, $J=7.8 \mathrm{~Hz}, 1 \mathrm{H}), 6.99$ (dd, $J=8.5,7.8 \mathrm{~Hz}, 1 \mathrm{H}), 7.00$ (d, $J=8.5 \mathrm{~Hz}, 1 \mathrm{H}), 6.28$ (dd, $J=2.1,1.5 \mathrm{~Hz}, 1 \mathrm{H}), 5.78(\mathrm{dd}, J=9.1,6.2 \mathrm{~Hz}, 1 \mathrm{H}), 3.47$ (dd, $J=16.0,9.1 \mathrm{~Hz}, 1 \mathrm{H}), 3.06$ (dd, $J=16.0$, $6.2 \mathrm{~Hz}, 1 \mathrm{H}), 1.36(\mathrm{~s}, 9 \mathrm{H}) ;{ }^{13} \mathrm{C}\left\{{ }^{1} \mathrm{H}\right\} \mathrm{NMR}\left(126 \mathrm{MHz}, \mathrm{CDCL}_{3}\right) \delta$ 169.3, 163.0 (d, J=247.3 Hz, 1C), 142.5 (d, $J=6.4 \mathrm{~Hz}, 1 \mathrm{C}$ ), 139.7, 130.4 (d, $J=8.3 \mathrm{~Hz}, 1 \mathrm{C}), 129.2,122.4$ (d, $J=2.8 \mathrm{~Hz}, 1 \mathrm{C}$ ), 115.2 (d, $J=21.1 \mathrm{~Hz}, 1 \mathrm{C}), 114.0(\mathrm{~d}, J=22.1 \mathrm{~Hz}, 1 \mathrm{C}), 105.8,81.5,61.7,41.7,28.0(3 \mathrm{C}) ;{ }^{19} \mathrm{~F}$ NMR $(471 \mathrm{MHz}$, $\left.\mathrm{CDCl}_{3}\right) \delta-112.12(\mathrm{td}, J=8.5,5.5 \mathrm{~Hz}, 1 \mathrm{~F})$; UPLC: (Acquity BEH C18, $\mathrm{MeCN} / \mathrm{H}_{2} \mathrm{O} / \mathrm{TFA}=$ $5 / 95 / 0.05 \rightarrow 95 / 5 / 0.05$, flow rate $=1.0 \mathrm{~mL} / \mathrm{min}, \lambda=220 \mathrm{~nm}$ ) $\mathrm{tR}=1.30 \mathrm{~min}$; HRMS (ESI) $\mathrm{m} / \mathrm{z}$ : $[\mathrm{M}+\mathrm{H}]^{+}$calcd for $\mathrm{C}_{16} \mathrm{H}_{20} \mathrm{~N}_{2} \mathrm{O}_{2} \mathrm{~F} 291.1509$; found 291.1503. 


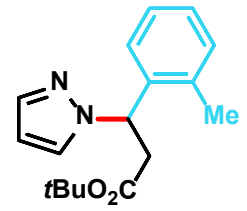

Substrate 7e: The standard procedure was followed with pyrazole $(68 \mathrm{mg}, 1.0$ mmol) and tert-butyl (E)-3-(o-tolyl)acrylate $(218 \mathrm{mg}, 1.0 \mathrm{mmol})$ to afford, after column chromatography $(10 \rightarrow 50 \%$ EtOAc/hexanes), 7 e $(250 \mathrm{mg}, 0.9 \mathrm{mmol}$, $87 \%)$ as a pale yellow oil.

${ }^{1} \mathrm{H}$ NMR $\left(500 \mathrm{MHz}, \mathrm{CDCl}_{3}\right) \delta 7.52(\mathrm{~d}, J=1.8 \mathrm{~Hz}, 1 \mathrm{H}), 7.29(\mathrm{~d}, J=2.5 \mathrm{~Hz}, 1 \mathrm{H}), 7.30-7.27(\mathrm{~m}, 1 \mathrm{H})$, 7.22 - 7.15 (m, 3H), 6.20 (dd, $J=2.5,1.8 \mathrm{~Hz}, 1 \mathrm{H}), 6.04$ (dd, $J=8.8,6.6 \mathrm{~Hz}, 1 \mathrm{H}$ ), 3.43 (dd, $J=15.9$, $8.9 \mathrm{~Hz}, 1 \mathrm{H}), 2.99$ (dd, $J=15.9,6.5 \mathrm{~Hz}, 1 \mathrm{H}), 2.37$ (s, 3H), $1.32(\mathrm{~s}, 9 \mathrm{H}) ;{ }^{13} \mathrm{C}\left\{{ }^{1} \mathrm{H}\right\} \mathrm{NMR}(126 \mathrm{MHz}$, $\left.\mathrm{CDCl}_{3}\right) \delta 169.6,139.3,137.4,135.9,130.9,128.8,128.3,126.6,126.5,105.5,81.2,58.6,41.2$, 28.0 (3C), 19.3; UPLC (Acquity BEH C18, $\mathrm{MeCN} / \mathrm{H}_{2} \mathrm{O} / \mathrm{TFA}=5 / 95 / 0.05 \rightarrow 95 / 5 / 0.05$, flow rate $=1.0 \mathrm{~mL} / \mathrm{min}, \lambda=220 \mathrm{~nm}$ ) $\mathrm{tR}=1.34 \mathrm{~min}$; HRMS (ESI) $\mathrm{m} / \mathrm{z}:[\mathrm{M}+\mathrm{H}]^{+}$calcd for $\mathrm{C}_{17} \mathrm{H}_{23} \mathrm{~N}_{2} \mathrm{O}_{2}$ 287.1760; found 287.1752 .

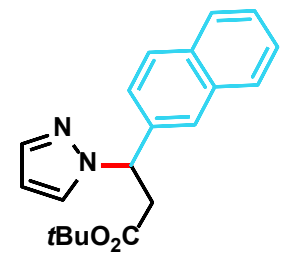

Substrate 7f: The standard procedure was followed with pyrazole (68 mg, 1.0 $\mathrm{mmol})$ and tert-butyl (E)-3-(naphthalen-2yl)acrylate $(254 \mathrm{mg}, 1.0 \mathrm{mmol})$ to afford, after column chromatography ( $10 \rightarrow 50 \%$ EtOAc/hexanes), $7 \mathbf{f}(243 \mathrm{mg}$, $0.8 \mathrm{mmol}, 75 \%)$ as a white solid.

${ }^{1} \mathrm{H}$ NMR (500 MHz, $\left.\mathrm{CDCl}_{3}\right) \delta 7.81(\mathrm{~s}, 1 \mathrm{H}), 7.81-7.78(\mathrm{~m}, 2 \mathrm{H}), 7.75(\mathrm{~d}, J=2.0 \mathrm{~Hz}, 1 \mathrm{H}), 7.58$ (d, $J=1.5 \mathrm{~Hz}, 1 \mathrm{H}), 7.50-7.45(\mathrm{~m}, 3 \mathrm{H}), 7.41$ (dd, $J=8.5,1.7 \mathrm{~Hz}, 1 \mathrm{H}), 6.25$ (dd, $J=2.0,1.6 \mathrm{~Hz}, 1 \mathrm{H})$, 5.95 (dd, $J=9.1,6.3 \mathrm{~Hz}, 1 \mathrm{H}), 3.58$ (dd, $J=15.9,9.1 \mathrm{~Hz}, 1 \mathrm{H}), 3.16$ (dd, $J=15.9,6.3 \mathrm{~Hz}, 1 \mathrm{H}), 1.34$ (s, $9 \mathrm{H}) ;{ }^{13} \mathrm{C}\left\{{ }^{1} \mathrm{H}\right\}$ NMR $\left(126 \mathrm{MHz}, \mathrm{CDCl}_{3}\right) \delta 169.6,139.5,137.3,133.3,133.1,129.2,128.8,128.2$, 127.8, 126.5, 126.4, 125.9, 124.7, 105.7, 81.3, 62.4, 41.8, 28.0 (3C); UPLC (Acquity BEH C18, $\mathrm{MeCN} / \mathrm{H}_{2} \mathrm{O} / \mathrm{TFA}=5 / 95 / 0.05 \rightarrow 95 / 5 / 0.05$, flow rate $\left.=1.0 \mathrm{~mL} / \mathrm{min}, \lambda=220 \mathrm{~nm}\right) \mathrm{tR}=1.39 \mathrm{~min}$; HRMS (ESI) $\mathrm{m} / \mathrm{z}$ : $[\mathrm{M}+\mathrm{H}]^{+}$calcd for $\mathrm{C}_{20} \mathrm{H}_{23} \mathrm{~N}_{2} \mathrm{O}_{2}$ 323.1760; found 323.1754 .

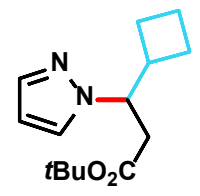

Substrate 7g: The standard procedure was followed with pyrazole $(75 \mathrm{mg}, 1.1 \mathrm{mmol})$ and tert-butyl (E)-3-cyclobutylacrylate $(200 \mathrm{mg}, 1.1 \mathrm{mmol})$ to afford, after column chromatography ( $10 \rightarrow 50 \%$ EtOAc/hexanes), $7 \mathbf{g}(230 \mathrm{mg}, 0.9 \mathrm{mmol}, 84 \%)$ as a colorless oil.

${ }^{1} \mathrm{H}$ NMR (600 MHz, CDCl$) \delta 7.48$ (t, $\left.J=1.8 \mathrm{~Hz}, 1 \mathrm{H}\right), 7.38$ (t, $\left.J=1.8 \mathrm{~Hz}, 1 \mathrm{H}\right), 6.15$ (q, $J=1.8 \mathrm{~Hz}$, $1 \mathrm{H}), 4.45$ (tdd, $J=9.7,3.8,1.8 \mathrm{~Hz}, 1 \mathrm{H}), 2.85-2.81$ (m, 1H), 2.80 (ddd, $J=15.8,9.7,1.2 \mathrm{~Hz}, 1 \mathrm{H}$ ), 
2.59 (ddd, $J=15.8,4.1,1.2 \mathrm{~Hz}, 1 \mathrm{H}), 2.10-2.03(\mathrm{~m}, 1 \mathrm{H}), 1.87$ - $1.73(\mathrm{~m}, 4 \mathrm{H}), 1.71-1.66(\mathrm{~m}, 1 \mathrm{H})$, $1.30(\mathrm{~s}, 9 \mathrm{H}) ;{ }^{13} \mathrm{C}\left\{{ }^{1} \mathrm{H}\right\}$ NMR $\left(151 \mathrm{MHz}, \mathrm{CDCl}_{3}\right) \delta 170.3,139.3,129.5,104.4,80.9,64.2,39.8$, 38.7, 28.0 (3C), 25.7, 25.6, 17.5; UPLC (Acquity BEH C18, $\mathrm{MeCN} / \mathrm{H}_{2} \mathrm{O} / \mathrm{TFA}=5 / 95 / 0.05 \rightarrow$ $95 / 5 / 0.05$, flow rate $=1.0 \mathrm{~mL} / \mathrm{min}, \lambda=220 \mathrm{~nm}) \mathrm{tR}=1.26 \mathrm{~min} ; \mathrm{HRMS}(\mathrm{ESI}) \mathrm{m} / \mathrm{z}:[\mathrm{M}+\mathrm{H}]^{+}$calcd for $\mathrm{C}_{14} \mathrm{H}_{23} \mathrm{~N}_{2} \mathrm{O}_{2} 251.1760$; found 251.1751.

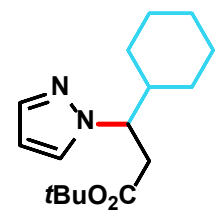

Substrate 7h: The standard procedure was followed with pyrazole $(68 \mathrm{mg}, 1.0$ $\mathrm{mmol})$ and tert-butyl (E)-3-cyclohexylacrylate $(210 \mathrm{mg}, 1.0 \mathrm{mmol})$ to afford, after column chromatography $(10 \rightarrow 50 \%$ EtOAc/hexanes $), 7 \mathbf{h}(237 \mathrm{mg}, 0.9 \mathrm{mmol}, 85 \%)$ as a colorless oil.

${ }^{1} \mathrm{H}$ NMR $\left(500 \mathrm{MHz}, \mathrm{CDCl}_{3}\right) \delta 7.47$ (d, $\left.J=1.2 \mathrm{~Hz}, 1 \mathrm{H}\right), 7.33$ (d, $\left.J=2.1 \mathrm{~Hz}, 1 \mathrm{H}\right), 6.13$ (dd, $J=2.2$, $1.2 \mathrm{~Hz}, 1 \mathrm{H}), 4.24$ (ddd, $J=10.1,9.1,4.4 \mathrm{~Hz}, 1 \mathrm{H}), 2.90$ (dd, $J=15.7,10.1 \mathrm{~Hz}, 1 \mathrm{H}), 2.76$ (dd, $J=15.7,4.4 \mathrm{~Hz}, 1 \mathrm{H}), 1.84-1.70$ (m, 3H), 1.66 - 1.57 (m, 2H), 1.27 (s, 9H), 1.24 - 1.04 (m, 4H), $1.00-0.90(\mathrm{~m}, 1 \mathrm{H}), 0.87-0.78(\mathrm{~m}, 1 \mathrm{H}) ;{ }^{13} \mathrm{C}\left\{{ }^{1} \mathrm{H}\right\} \mathrm{NMR}\left(126 \mathrm{MHz}, \mathrm{CDCl}_{3}\right) \delta 170.6,139.2$, 130.1, 104.2, 80.8, 64.4, 42.5, 38.9, 30.1, 29.4, 27.9 (3C), 26.2, 26.1, 25.8; UPLC (Acquity BEH $\mathrm{C} 18, \mathrm{MeCN} / \mathrm{H}_{2} \mathrm{O} / \mathrm{TFA}=5 / 95 / 0.05 \rightarrow 95 / 5 / 0.05$, flow rate $\left.=1.0 \mathrm{~mL} / \mathrm{min}, \lambda=220 \mathrm{~nm}\right) \mathrm{tR}=1.36$ min; HRMS (ESI) m/z: [M+H] calcd for $\mathrm{C}_{16} \mathrm{H}_{2} 7 \mathrm{~N}_{2} \mathrm{O}_{2} 279.2073$; found 279.2067.

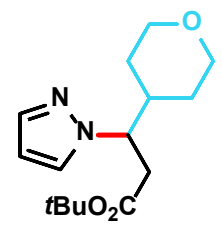

Substrate 7i: The standard procedure was followed with pyrazole $(68 \mathrm{mg}, 1.0$ mmol) and tert-butyl (E)-3-(tetrahydro-2H-pyran-4-yl)propanoate (212 mg, 1.0 mmol) to afford, after column chromatography $(10 \rightarrow 50 \%$ EtOAc/hexanes $), 7 \mathbf{i}$ (232 $\mathrm{mg}, 0.8 \mathrm{mmol}, 83 \%$ ) as a colorless oil.

${ }^{1} \mathrm{H}$ NMR (600 MHz, CDCl$) \delta 7.50(\mathrm{~d}, J=1.8 \mathrm{~Hz}, 1 \mathrm{H}), 7.37(\mathrm{~d}, J=1.8 \mathrm{~Hz}, 1 \mathrm{H}), 6.16(\mathrm{t}, J=1.8 \mathrm{~Hz}$, 1H), 4.26 (td, $J=9.5,4.1 \mathrm{~Hz}, 1 \mathrm{H}), 3.98$ (dd, $J=11.4,3.9 \mathrm{~Hz}, 1 \mathrm{H}), 3.87$ (dd, $J=11.4,4.0 \mathrm{~Hz}, 1 \mathrm{H}$ ), 3.35 (t, $J=11.8 \mathrm{~Hz}, 1 \mathrm{H}), 3.26$ (t, $J=11.8 \mathrm{~Hz}, 1 \mathrm{H}), 2.92$ (dd, $J=15.7,9.5 \mathrm{~Hz}, 1 \mathrm{H}), 2.77$ (dd, $J=15.7$, $4.1 \mathrm{~Hz}, 1 \mathrm{H}), 2.14-2.06$ (m, 1H), 1.64 (d, J=13.0 Hz, 1H), $1.41-1.34$ (m, 1H), 1.29 (s, 9H), 1.27 - $1.18(\mathrm{~m}, 1 \mathrm{H}), 0.98(\mathrm{~d}, J=13.0 \mathrm{~Hz}, 1 \mathrm{H}) ;{ }^{13} \mathrm{C}\left\{{ }^{1} \mathrm{H}\right\} \mathrm{NMR}\left(151 \mathrm{MHz}, \mathrm{CDCl}_{3}\right) \delta 170.3,139.7,130.5$, 104.4, 81.1, 67.7, 67.5, 63.9, 40.0, 38.5, 30.1, 29.6, 28.0 (3C); UPLC (Acquity BEH C18, $\mathrm{MeCN} / \mathrm{H}_{2} \mathrm{O} / \mathrm{TFA}=5 / 95 / 0.05 \rightarrow 95 / 5 / 0.05$, flow rate $\left.=1.0 \mathrm{~mL} / \mathrm{min}, \lambda=220 \mathrm{~nm}\right) \mathrm{tR}=1.11 \mathrm{~min}$; HRMS (ESI) m/z: [M+H] calcd for $\mathrm{C}_{15} \mathrm{H}_{25} \mathrm{~N}_{2} \mathrm{O}_{3} 281.1865$; found 281.1858 . 


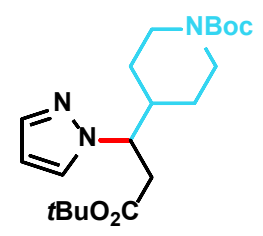

Substrate 7j: The standard procedure was followed with pyrazole $(35 \mathrm{mg}, 0.5$ mmol) and tert-butyl (E)-4-(3-(tert-butoxy)-3-oxoprop-1-en-1-yl)piperidine-1carboxylate $(160 \mathrm{mg}, 0.5 \mathrm{mmol})$ to afford, after column chromatography $(10 \rightarrow$ $50 \%$ EtOAc/hexanes), $7 \mathbf{j}$ (183 mg, $0.5 \mathrm{mmol}, 94 \%$ ) as a colorless oil.

Note: NMRs are broadened here likely due to the presence of rotomers. ${ }^{1} \mathrm{H}$ NMR $(500 \mathrm{MHz}$, $\left.\mathrm{CDCl}_{3}\right) \delta 7.52$ - $7.48(\mathrm{~m}, 1 \mathrm{H}), 7.37$ - $7.33(\mathrm{~m}, 1 \mathrm{H}), 6.18$ - $6.14(\mathrm{~m}, 1 \mathrm{H}), 4.30$ - $4.23(\mathrm{~m}, 1 \mathrm{H}), 4.21$ - $4.08(\mathrm{~m}, 1 \mathrm{H}), 4.07-3.95(\mathrm{~m}, 1 \mathrm{H}), 2.99-2.89(\mathrm{~m}, 1 \mathrm{H}), 2.81$ - $2.73(\mathrm{~m}, 1 \mathrm{H}), 2.71$ - $2.61(\mathrm{~m}, 1 \mathrm{H})$, $2.60-2.50(\mathrm{~m}, 1 \mathrm{H}), 2.06-1.95(\mathrm{~m}, 1 \mathrm{H}), 1.76-1.67(\mathrm{~m}, 1 \mathrm{H}), 1.43-1.39(\mathrm{~m}, 9 \mathrm{H}), 1.31$ - $1.27(\mathrm{~m}$, 9H), 1.23 - $1.13(\mathrm{~m}, 1 \mathrm{H}), 1.10-1.02(\mathrm{~m}, 2 \mathrm{H}) ;{ }^{13} \mathrm{C}\left\{{ }^{1} \mathrm{H}\right\} \mathrm{NMR}\left(126 \mathrm{MHz}, \mathrm{CDCl}_{3}\right) \delta$ 170.2, 154.7, 139.7, 130.4, 104.5, 81.1, 79.5, 63.6, 43.6 (br, 2C), ${ }^{*} 41.0,38.7,29.2$ (br), 28.7 (br), 28.5 (3C), 27.9 (3C), "assignment corroborated by HSQC; UPLC (Acquity BEH C18, MeCN/ $\mathrm{H}_{2} \mathrm{O} / \mathrm{TFA}=$ $5 / 95 / 0.05 \rightarrow 95 / 5 / 0.05$, flow rate $=1.0 \mathrm{~mL} / \mathrm{min}, \lambda=220 \mathrm{~nm}$ ) $\mathrm{tR}=1.30 \mathrm{~min}$; HRMS (ESI) $\mathrm{m} / \mathrm{z}$ : $[\mathrm{M}+\mathrm{H}]^{+}$calcd for $\mathrm{C}_{20} \mathrm{H}_{34} \mathrm{~N}_{3} \mathrm{O}_{4} 380.2549$; found 380.2541 .

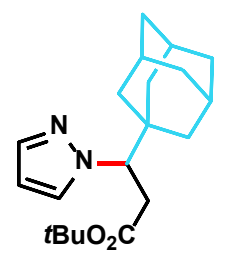

Substrate 7k: In a departure from the standard procedure, pyrazole (136 mg, 2.0 mmol, 2.0 equiv), tert-butyl (E)-3-(adamantan-1-yl)acrylate ( $262 \mathrm{mg}, 1.0 \mathrm{mmol}$, 1.0 equiv), and potassium tert-butoxide ( $73 \mathrm{mg}, 1.0 \mathrm{mmol}, 1.0$ equiv) were combined in $\mathrm{MeCN}(1.0 \mathrm{~mL})$. The reaction mixture was heated at $80{ }^{\circ} \mathrm{C}$ for 30 $\min , 60^{\circ} \mathrm{C}$ for $1.5 \mathrm{~h}$, and then left to stir at room temperature for $14 \mathrm{~h}$. The crude residue was purified by column chromatography $(0 \rightarrow 25 \%$ EtOAc/hexanes) to afford $7 \mathbf{k}$ ( $235 \mathrm{mg}, 0.7 \mathrm{mmol}$, $71 \%)$ as a white solid.

${ }^{1} \mathrm{H}$ NMR (600 MHz, CDCl 3$) \delta 7.48$ (d, $\left.J=1.9 \mathrm{~Hz}, 1 \mathrm{H}\right), 7.34$ (d, $\left.J=1.9 \mathrm{~Hz}, 1 \mathrm{H}\right), 6.17$ (t, $J=1.9 \mathrm{~Hz}$, 1H), 4.15 (dd, $J=11.4,3.4 \mathrm{~Hz}, 1 \mathrm{H}), 3.08$ (dd, $J=15.7,11.4 \mathrm{~Hz}, 1 \mathrm{H}), 2.73$ (dd, $J=15.7,3.4 \mathrm{~Hz}$, 1H), 1.97 (br s, 3H), 1.67 (br d, $J=12.0 \mathrm{~Hz}, 6 \mathrm{H}$ ), 1.58 (br d, $J=12.0 \mathrm{~Hz}, 3 \mathrm{H}$ ), 1.37 (br d, $J=12.0$ $\mathrm{Hz}, 3 \mathrm{H}), 1.26$ (s, 9H); ${ }^{13} \mathrm{C}\left\{{ }^{1} \mathrm{H}\right\} \mathrm{NMR}\left(151 \mathrm{MHz}, \mathrm{CDCl}_{3}\right) \delta$ 171.1, 138.7, 131.1, 104.0, 80.8, 68.6, 39.2 (3C), 36.91 (3C), 36.86, 34.8, 28.5 (3C), 28.0 (3C); UPLC (Acquity BEH C18, $\mathrm{MeCN} / \mathrm{H}_{2} \mathrm{O} / \mathrm{TFA}=5 / 95 / 0.05 \rightarrow 95 / 5 / 0.05$, flow rate $\left.=1.0 \mathrm{~mL} / \mathrm{min}, \lambda=220 \mathrm{~nm}\right) \mathrm{tR}=1.57 \mathrm{~min}$; HRMS (ESI) m/z: [M+H] $]^{+}$calcd for $\mathrm{C}_{20} \mathrm{H}_{31} \mathrm{~N}_{2} \mathrm{O}_{2}$ 331.2386; found 331.2381. 


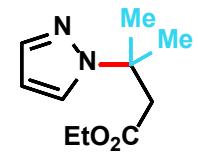

Substrate 71: The standard procedure was followed with pyrazole (68 mg, 1.0 $\mathrm{mmol}$ ) and ethyl 3-methylbut-2-enoate $(128 \mathrm{mg}, 1.0 \mathrm{mmol})$ to afford, after column chromatography ( $10 \rightarrow 50 \%$ EtOAc/hexanes), 71 (156 mg, $0.8 \mathrm{mmol}, 80 \%)$ as a colorless oil.

${ }^{1} \mathrm{H}$ NMR (500 MHz, $\left.\mathrm{CDCl}_{3}\right) \delta 7.47$ (d, $\left.J=2.1 \mathrm{~Hz}, 2 \mathrm{H}\right), 6.16(\mathrm{t}, J=2.1 \mathrm{~Hz}, 1 \mathrm{H}), 3.96$ (q, $J=7.1 \mathrm{~Hz}$, 2H), $2.85(\mathrm{~s}, 2 \mathrm{H}), 1.67(\mathrm{~s}, 6 \mathrm{H}), 1.09(\mathrm{t}, J=7.1 \mathrm{~Hz}, 3 \mathrm{H}) ;{ }^{13} \mathrm{C}\left\{{ }^{1} \mathrm{H}\right\} \mathrm{NMR}\left(126 \mathrm{MHz}, \mathrm{CDC}_{3}\right) \delta$ 170.1, 138.9, 126.2, 104.6, 60.2, 58.9, 46.6, 27.7 (3C), 14.0 (2C); UPLC (Acquity BEH C18, $\mathrm{MeCN} / \mathrm{H}_{2} \mathrm{O} / \mathrm{NH}_{4} \mathrm{OAc}=5 / 95 / 10 \mathrm{mM} \rightarrow 95 / 5 / 10 \mathrm{mM}$, flow rate $\left.=1.0 \mathrm{~mL} / \mathrm{min}, \lambda=220 \mathrm{~nm}\right) \mathrm{tR}=$ $0.97 \mathrm{~min}$; HRMS (ESI) m/z: $[\mathrm{M}+\mathrm{H}]^{+}$calcd for $\mathrm{C}_{10} \mathrm{H}_{17} \mathrm{~N}_{2} \mathrm{O}_{2}$ 197.1290; found 197.1286.

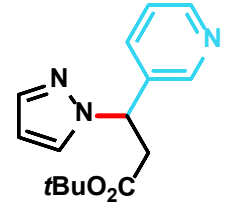

Substrate 7m: The standard procedure was followed with pyrazole $(68 \mathrm{mg}, 1.0$ mmol) and tert-butyl (E)-3-(pyridin-3-yl)acrylate (205 mg, $1.0 \mathrm{mmol}$ ) to afford, after column chromatography $(10 \rightarrow 50 \%$ EtOAc/hexanes $), 7 m(197 \mathrm{mg}, 0.7$ mmol, $72 \%$ ) as a colorless oil.

${ }^{1} \mathrm{H}$ NMR $\left(500 \mathrm{MHz}, \mathrm{CDCl}_{3}\right) \delta 8.57(\mathrm{~d}, J=2.3 \mathrm{~Hz}, 1 \mathrm{H}), 8.52(\mathrm{dd}, J=4.8,1.6 \mathrm{~Hz}, 1 \mathrm{H}), 7.64$ (ddd, $J=8.0,2.3,1.6 \mathrm{~Hz}, 1 \mathrm{H}), 7.54(\mathrm{~d}, J=1.7 \mathrm{~Hz}, 1 \mathrm{H}), 7.44(\mathrm{~d}, J=2.3 \mathrm{~Hz}, 1 \mathrm{H}), 7.24$ (dd, $J=8.0,4.8 \mathrm{~Hz}$, 1H), 6.24 (dd, $J=2.3,1.7 \mathrm{~Hz}, 1 \mathrm{H}$ ), 5.77 (dd, $J=9.0,6.4 \mathrm{~Hz}, 1 \mathrm{H}$ ), 3.46 (dd, $J=16.0,9.0 \mathrm{~Hz}, 1 \mathrm{H}$ ), $3.06(\mathrm{dd}, J=16.0,6.4 \mathrm{~Hz}, 1 \mathrm{H}), 1.32(\mathrm{~s}, 9 \mathrm{H}) ;{ }^{13} \mathrm{C}\left\{{ }^{1} \mathrm{H}\right\} \mathrm{NMR}\left(126 \mathrm{MHz}, \mathrm{CDCl}_{3}\right) \delta$ 169.0, 149.7, 148.5, 139.9, 135.5, 134.5, 129.3, 123.7, 105.9, 81.7, 60.0, 41.6, 28.0 (3C); UPLC (Acquity BEH $\mathrm{C} 18, \mathrm{MeCN} / \mathrm{H}_{2} \mathrm{O} / \mathrm{TFA}=5 / 95 / 0.05 \rightarrow 95 / 5 / 0.05$, flow rate $\left.=1.0 \mathrm{~mL} / \mathrm{min}, \lambda=220 \mathrm{~nm}\right) \mathrm{tR}=0.86$ min; HRMS (ESI) m/z: [M+H] $]^{+}$calcd for $\mathrm{C}_{15} \mathrm{H}_{20} \mathrm{~N}_{3} \mathrm{O}_{2} 274$.1556; found 274.1546.

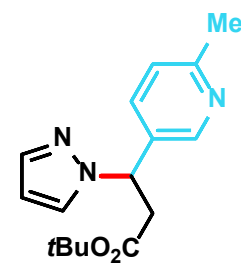

Substrate 7n: The standard procedure was followed with pyrazole (68 mg, 1.0 mmol) and tert-butyl (E)-3-(6-methylpyridin-3-yl)acrylate (219 mg, $1.0 \mathrm{mmol})$ to afford, after column chromatography $(10 \rightarrow 50 \%$ EtOAc/hexanes), $7 \mathbf{n}$ (235 $\mathrm{mg}, 0.8 \mathrm{mmol}, 82 \%)$ as a colorless liquid, containing a small amount ( $\mathrm{ca} .5 \%$ ) of dissolved 4, which was inseparable.

${ }^{1} \mathrm{H}$ NMR $\left(600 \mathrm{MHz}, \mathrm{CDCl}_{3}\right) \delta 8.45(\mathrm{~d}, J=2.2 \mathrm{~Hz}, 1 \mathrm{H}), 7.54(\mathrm{dd}, J=8.2,2.2 \mathrm{~Hz}, 1 \mathrm{H}), 7.53(\mathrm{~d}$, $J=1.6 \mathrm{~Hz}, 1 \mathrm{H}), 7.42$ (d, $J=2.0 \mathrm{~Hz}, 1 \mathrm{H}), 7.10$ (d, $J=8.2 \mathrm{~Hz}, 1 \mathrm{H}), 6.23$ (dd, $J=2.0,1.6 \mathrm{~Hz}, 1 \mathrm{H}), 5.74$ (dd, $J=8.7,6.5 \mathrm{~Hz}, 1 \mathrm{H}$ ), 3.44 (dd, $J=15.9,9.0 \mathrm{~Hz}, 1 \mathrm{H}), 3.03$ (dd, $J=16.0,6.4 \mathrm{~Hz}, 1 \mathrm{H}), 2.51$ (s, 3H), $1.32(\mathrm{~s}, 9 \mathrm{H}) ;{ }^{13} \mathrm{C}\left\{{ }^{1} \mathrm{H}\right\}$ NMR (126 MHz, $\left.\mathrm{CDCl}_{3}\right) \delta 169.1,158.6,147.7,139.8,134.9,132.4$, 
129.1, 123.4, 105.8, 81.6, 59.8, 41.6, 28.0 (3C), 24.2; UPLC (Acquity BEH C18, $\mathrm{MeCN} / \mathrm{H}_{2} \mathrm{O} / \mathrm{TFA}=5 / 95 / 0.05 \rightarrow 95 / 5 / 0.05$, flow rate $\left.=1.0 \mathrm{~mL} / \mathrm{min}, \lambda=220 \mathrm{~nm}\right) \mathrm{tR}=0.86 \mathrm{~min}$; HRMS (ESI) m/z: [M+H] $]^{+}$calcd for $\mathrm{C}_{16} \mathrm{H}_{22} \mathrm{~N}_{3} \mathrm{O}_{2} 288.1712$; found 288.1704 .

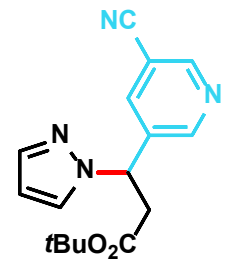

Substrate 7o: The standard procedure was followed with pyrazole $(68 \mathrm{mg}, 1.0$ mmol) and tert-butyl (E)-3-(5-cyanopyridin-3-yl)acrylate (230 mg, $1.0 \mathrm{mmol})$ to afford, after column chromatography ( $10 \rightarrow 50 \%$ EtOAc/hexanes), $7 \mathbf{o}(235 \mathrm{mg}$, $0.8 \mathrm{mmol}, 79 \%$ ) as a yellow oil.

${ }^{1} \mathrm{H}$ NMR $\left(600 \mathrm{MHz}, \mathrm{CDCl}_{3}\right) \delta 8.79(\mathrm{~d}, J=1.6 \mathrm{~Hz}, 1 \mathrm{H}), 8.77$ (d, $\left.J=2.0 \mathrm{~Hz}, 1 \mathrm{H}\right), 7.95$ (dd, $J=2.0$, $1.6 \mathrm{~Hz}, 1 \mathrm{H}), 7.58$ (d, $J=1.6 \mathrm{~Hz}, 1 \mathrm{H}), 7.49$ (d, $J=2.0 \mathrm{~Hz}, 1 \mathrm{H}), 6.28$ (dd, $J=2.0,1.6 \mathrm{~Hz}, 1 \mathrm{H}), 5.81$ (dd, $J=8.4,6.7 \mathrm{~Hz}, 1 \mathrm{H}), 3.44$ (dd, $J=16.2,8.7 \mathrm{~Hz}, 1 \mathrm{H}), 3.09$ (dd, $J=16.3,6.4 \mathrm{~Hz}, 1 \mathrm{H}), 1.34$ (s, $9 \mathrm{H}) ;{ }^{13} \mathrm{C}\left\{{ }^{1} \mathrm{H}\right\} \mathrm{NMR}\left(151 \mathrm{MHz}, \mathrm{CDCl}_{3}\right) \delta 168.6,152.0,151.7,140.7,137.9,136.3,129.6,116.3$, 110.2, 106.4, 82.2, 59.1, 41.5, 28.0 (3C); UPLC (Acquity BEH C18, $\mathrm{MeCN} / \mathrm{H}_{2} \mathrm{O} / \mathrm{TFA}=$ $5 / 95 / 0.05 \rightarrow 95 / 5 / 0.05$, flow rate $=1.0 \mathrm{~mL} / \mathrm{min}, \lambda=220 \mathrm{~nm}$ ) $\mathrm{tR}=1.11 \mathrm{~min} ; \mathrm{HRMS}(\mathrm{ESI}) \mathrm{m} / \mathrm{z}$ : $[\mathrm{M}+\mathrm{H}]^{+}$calcd for $\mathrm{C}_{16} \mathrm{H}_{19} \mathrm{~N}_{4} \mathrm{O}_{2} 299.1508$; found 299.1500 .

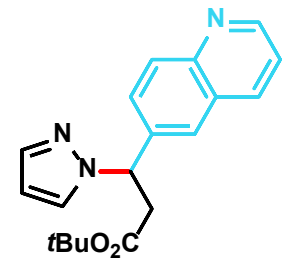

Substrate 7p: The standard procedure was followed with pyrazole (68 mg, $1.0 \mathrm{mmol}$ ) and tert-butyl (E)-3-(quinolin-6-yl)acrylate $(255 \mathrm{mg}, 1.0 \mathrm{mmol})$ to afford, after column chromatography $(10 \rightarrow 50 \%$ EtOAc/hexanes), $7 \mathbf{p}$ (280 $\mathrm{mg}, 0.9 \mathrm{mmol}, 87 \%)$ as a colorless liquid, containing a small amount $(<10 \%)$ of $\mathbf{4}$, which was inseparable.

${ }^{1} \mathrm{H}$ NMR $\left(600 \mathrm{MHz}, \mathrm{CDCl}_{3}\right) \delta 8.89(\mathrm{dd}, J=4.2,1.7 \mathrm{~Hz}, 1 \mathrm{H}), 8.10(\mathrm{~d}, J=8.2 \mathrm{~Hz}, 1 \mathrm{H}), 8.06$ (d, $J=8.8 \mathrm{~Hz}, 1 \mathrm{H}), 7.71(\mathrm{~s}, 1 \mathrm{H}), 7.64(\mathrm{dd}, J=8.8,1.7 \mathrm{~Hz}, 1 \mathrm{H}), 7.57$ (d, $J=1.6 \mathrm{~Hz}, 1 \mathrm{H}), 7.48(\mathrm{~d}, J=2.0$ $\mathrm{Hz}, 1 \mathrm{H}), 7.39$ (dd, $J=8.2,4.2 \mathrm{~Hz}, 1 \mathrm{H}), 6.26$ (dd, $J=2.0,1.6 \mathrm{~Hz}, 1 \mathrm{H}), 5.96$ (dd, $J=9.0,6.4 \mathrm{~Hz}, 1 \mathrm{H}$ ), 3.55 (dd, $J=16.0,9.0 \mathrm{~Hz}, 1 \mathrm{H}), 3.16(\mathrm{dd}, J=16.0,6.4 \mathrm{~Hz}, 1 \mathrm{H}), 1.32(\mathrm{~s}, 9 \mathrm{H}) ;{ }^{13} \mathrm{C}\left\{{ }^{1} \mathrm{H}\right\} \mathrm{NMR}(151$ $\left.\mathrm{MHz}, \mathrm{CDCl}_{3}\right) \delta 169.4,150.9,148.0,139.7,138.2,136.3,130.3,129.3,128.3,128.1,125.7$, 121.6, 105.9, 81.5, 62.0, 41.7, 28.0 (3C); UPLC (Acquity BEH C18, $\mathrm{MeCN} / \mathrm{H}_{2} \mathrm{O} / \mathrm{TFA}=$ $5 / 95 / 0.05 \rightarrow 95 / 5 / 0.05$, flow rate $=1.0 \mathrm{~mL} / \mathrm{min}, \lambda=220 \mathrm{~nm}$ ) $\mathrm{tR}=0.92 \mathrm{~min} ;$ HRMS (ESI) $\mathrm{m} / \mathrm{z}$ : $[\mathrm{M}+\mathrm{H}]^{+}$calc'd for $\mathrm{C}_{19} \mathrm{H}_{22} \mathrm{~N}_{3} \mathrm{O}_{2} 324.1712$; found 324.1703. 


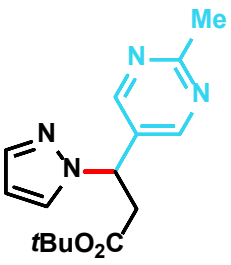

Substrate 7q: The standard procedure was followed with pyrazole (68 mg, 1.0 $\mathrm{mmol})$ and tert-butyl (E)-3-(2-methylpyrimidin-5-yl)acrylate (220 mg, $1.0 \mathrm{mmol})$ to afford, after column chromatography $(10 \rightarrow 50 \%$ EtOAc/hexanes $), 7 q$ ( $224 \mathrm{mg}$, $0.8 \mathrm{mmol}, 78 \%$ ) as a colorless oil.

${ }^{1} \mathrm{H}$ NMR (500 MHz, $\left.\mathrm{CDCl}_{3}\right) \delta 8.60$ (s, 2H), 7.54 (d, $\left.J=1.6 \mathrm{~Hz}, 1 \mathrm{H}\right), 7.46(\mathrm{~d}, J=2.3 \mathrm{~Hz}, 1 \mathrm{H}), 6.24$ (dd, $J=2.3,1.6 \mathrm{~Hz}, 1 \mathrm{H}), 5.72$ (dd, $J=8.7,6.6 \mathrm{~Hz}, 1 \mathrm{H}), 3.44$ (dd, $J=16.1,8.7 \mathrm{~Hz}, 1 \mathrm{H}), 3.07$ (dd, $J=16.1,6.6 \mathrm{~Hz}, 1 \mathrm{H}), 2.69$ (s, 3H), 1.33 (s, 9H); ${ }^{13} \mathrm{C}\left\{{ }^{1} \mathrm{H}\right\} \mathrm{NMR}\left(126 \mathrm{MHz}, \mathrm{CDCL}_{3}\right) \delta$ 168.8, 168.2, 155.8 (2C), 140.4, 130.0, 129.3, 106.1, 82.0, 58.0, 41.3, 28.0 (3C), 25.9; UPLC (Acquity BEH $\mathrm{C} 18, \mathrm{MeCN} / \mathrm{H}_{2} \mathrm{O} / \mathrm{TFA}=5 / 95 / 0.05 \rightarrow 95 / 5 / 0.05$, flow rate $\left.=1.0 \mathrm{~mL} / \mathrm{min}, \lambda=220 \mathrm{~nm}\right) \mathrm{tR}=1.00$ $\mathrm{min}$; HRMS (ESI) m/z: [M+H] $]^{+}$calcd for $\mathrm{C}_{15} \mathrm{H}_{21} \mathrm{~N}_{4} \mathrm{O}_{2} 289.1665$; found 289.1658 .

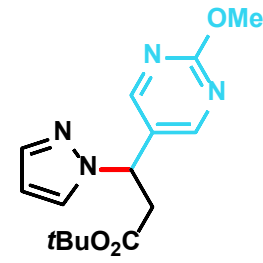

Substrate 7r: The standard procedure was followed with pyrazole (68 mg, 1.0 $\mathrm{mmol}$ ) and tert-butyl (E)-3-(2-methoxypyrimidin-5-yl)acrylate (236 mg, 1.0 mmol) to afford, after column chromatography $(10 \rightarrow 50 \%$ EtOAc/hexanes $), 7 \mathbf{r}$ (219 $\mathrm{mg}, 0.7 \mathrm{mmol}, 72 \%$ ) as a colorless oil.

${ }^{1} \mathrm{H}$ NMR (600 MHz, CDCl 3 ) $\delta 8.52(\mathrm{~s}, 2 \mathrm{H}), 7.55(\mathrm{~d}, J=1.6 \mathrm{~Hz}, 1 \mathrm{H}), 7.46(\mathrm{~d}, J=2.0 \mathrm{~Hz}, 1 \mathrm{H}), 6.25$ (dd, $J=2.0,1.6 \mathrm{~Hz}, 1 \mathrm{H}$ ), 5.70 (dd, $J=8.6,6.7 \mathrm{~Hz}, 1 \mathrm{H}$ ), 3.99 (s, 3H), 3.43 (dd, $J=16.1,8.6 \mathrm{~Hz}, 1 \mathrm{H}$ ), 3.07 (dd, $J=16.1,6.7 \mathrm{~Hz}, 1 \mathrm{H}), 1.35(\mathrm{~s}, 9 \mathrm{H}) ;{ }^{13} \mathrm{C}\left\{{ }^{1} \mathrm{H}\right\} \mathrm{NMR}\left(151 \mathrm{MHz}, \mathrm{CDCl}_{3}\right) \delta$ 168.9, 165.6, 158.3 (2C), 140.4, 129.2, 126.7, 106.1, 82.0, 57.7, 55.2, 41.5, 28.0 (3C); UPLC (Acquity BEH $\mathrm{C} 18, \mathrm{MeCN} / \mathrm{H}_{2} \mathrm{O} / \mathrm{TFA}=5 / 95 / 0.05 \rightarrow 95 / 5 / 0.05$, flow rate $\left.=1.0 \mathrm{~mL} / \mathrm{min}, \lambda=220 \mathrm{~nm}\right) \mathrm{tR}=1.09$ min; HRMS (ESI) m/z: [M+H] $]^{+}$calcd for $\mathrm{C}_{15} \mathrm{H}_{20} \mathrm{~N}_{4} \mathrm{O}_{3} 305.1614$; found 305.1609.

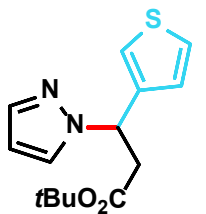

Substrate 7s: The standard procedure was followed with pyrazole $(68 \mathrm{mg}, 1.0$ $\mathrm{mmol}$ ) and tert-butyl (E)-3-(thiophen-3-yl)acrylate $(210 \mathrm{mg}, 1.0 \mathrm{mmol})$ to afford, after column chromatography $(10 \rightarrow 50 \%$ EtOAc/hexanes $), 7 \mathrm{~s}(230 \mathrm{mg}, 0.8 \mathrm{mmol}$, $89 \%)$ as a colorless oil.

${ }^{1} \mathrm{H}$ NMR $\left(600 \mathrm{MHz}, \mathrm{CDCl}_{3}\right) \delta 7.53(\mathrm{~d}, J=1.5 \mathrm{~Hz}, 1 \mathrm{H}), 7.41(\mathrm{~d}, J=2.4 \mathrm{~Hz}, 1 \mathrm{H}), 7.27(\mathrm{dd}, J=5.0,3.1$ $\mathrm{Hz}, 1 \mathrm{H}), 7.16(\mathrm{~d}, J=3.0 \mathrm{~Hz}, 1 \mathrm{H}), 7.00(\mathrm{~d}, J=5.0 \mathrm{~Hz}, 1 \mathrm{H}), 6.22(\mathrm{dd}, J=2.4,1.5 \mathrm{~Hz}, 1 \mathrm{H}), 5.86(\mathrm{dd}$, $J=8.7,6.4 \mathrm{~Hz}, 1 \mathrm{H}), 3.38$ (dd, $J=15.8,8.7 \mathrm{~Hz}, 1 \mathrm{H}), 3.06$ (dd, $J=15.8,6.4 \mathrm{~Hz}, 1 \mathrm{H}), 1.33$ (s, 9H); ${ }^{13} \mathrm{C}\left\{{ }^{1} \mathrm{H}\right\}$ NMR $\left(151 \mathrm{MHz}, \mathrm{CDCl}_{3}\right) \delta 169.4,140.7,139.6,128.9,126.6,126.4,122.4,105.4,81.3$, 58.2, 41.9, 28.0 (s, 3C); UPLC (Acquity BEH C18, $\mathrm{MeCN} / \mathrm{H}_{2} \mathrm{O} / \mathrm{TFA}=5 / 95 / 0.05 \rightarrow 95 / 5 / 0.05$, 
flow rate $=1.0 \mathrm{~mL} / \mathrm{min}, \lambda=220 \mathrm{~nm}) \mathrm{tR}=1.25 \mathrm{~min} ; \mathrm{HRMS}(\mathrm{ESI}) \mathrm{m} / \mathrm{z}:[\mathrm{M}+\mathrm{H}]^{+}$calc'd for $\mathrm{C}_{14} \mathrm{H}_{19} \mathrm{~N}-$ ${ }_{2} \mathrm{O}_{2} \mathrm{~S} 279.1167$; found 279.1160 .

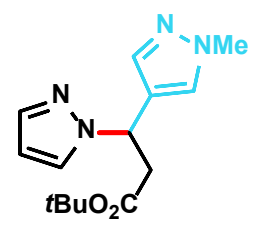

Substrate 7t: The standard procedure was followed with pyrazole $(68 \mathrm{mg}, 1.0$ mmol) and tert-butyl (E)-3-(1-methyl-1H-pyrazol-4-yl)acrylate (208 mg, 1.0 $\mathrm{mmol})$ to afford, after column chromatography $(10 \rightarrow 50 \%$ EtOAc/hexanes), $7 \mathbf{t}$ (209 mg, $0.8 \mathrm{mmol}, 76 \%$ ) as a colorless oil.

${ }^{1} \mathrm{H}$ NMR (600 MHz, CDCl $) \delta 7.51$ (br s, 1H), 7.42 (br s, 1H), 7.41 (br s, 1H), 7.31 (br s, 1H), 6.19 (br s, 1H), 5.75 (dd, J=8.9, 6.7 Hz, 1H), 3.83 (br s, 3H), 3.28 (dd, $J=16.0,8.9 \mathrm{~Hz}, 1 \mathrm{H}), 2.98$ (dd, $J=16.0,6.7 \mathrm{~Hz}, 1 \mathrm{H}), 1.33$ (s, 9H).; ${ }^{13} \mathrm{C}\left\{{ }^{1} \mathrm{H}\right\}$ NMR (151 MHz, $\left.\mathrm{CDCl}_{3}\right) \delta 169.3,139.5,137.5$, 128.63, 128.58, 120.9, 105.2, 81.2, 54.4, 42.4, 39.0, 27.9 (3C); UPLC (Acquity BEH C18, $\mathrm{MeCN} / \mathrm{H}_{2} \mathrm{O} / \mathrm{TFA}=5 / 95 / 0.05 \rightarrow 95 / 5 / 0.05$, flow rate $\left.=1.0 \mathrm{~mL} / \mathrm{min}, \lambda=220 \mathrm{~nm}\right) \mathrm{tR}=0.96 \mathrm{~min}$; MS (ESI) mz/: [M+H] calcd for $\mathrm{C}_{14} \mathrm{H}_{21} \mathrm{~N}_{4} \mathrm{O}_{2}$ 277.1665; found 277.1657. 


\section{Products Exploring Nucleophile Scope:}

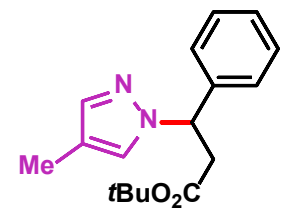

Substrate 8a: The standard procedue was followed with 4-methylpyrazole (82 $\mathrm{mg}, 1.0 \mathrm{mmol})$ and tert-butyl cinnamate $(204 \mathrm{mg}, 1.0 \mathrm{mmol})$ to afford, after column chromatography $(10 \rightarrow 50 \%$ EtOAc/hexanes $), 8 a(215 \mathrm{mg}, 0.8 \mathrm{mmol}$, $75 \%$ ) as a colorless oil.

${ }^{1} \mathrm{H}$ NMR (500 MHz, $\left.\mathrm{CDCl}_{3}\right) \delta 7.33(\mathrm{~s}, 1 \mathrm{H}), 7.32$ - $7.23(\mathrm{~m}, 5 \mathrm{H}), 7.17$ (q, J=0.7 Hz, 1H), 5.68 (dd, $J=9.0,6.4 \mathrm{~Hz}, 1 \mathrm{H}), 3.44$ (dd, $J=15.9,9.0 \mathrm{~Hz}, 1 \mathrm{H}), 3.01$ (dd, $J=15.9,6.4 \mathrm{~Hz}, 1 \mathrm{H}), 2.02$ (d, $J=0.7 \mathrm{~Hz}, 3 \mathrm{H}), 1.33(\mathrm{~s}, 9 \mathrm{H}) ;{ }^{13} \mathrm{C}\left\{{ }^{1} \mathrm{H}\right\} \mathrm{NMR}\left(126 \mathrm{MHz}, \mathrm{CDCl}_{3}\right) \delta 169.5,140.0,139.7,128.7$

(2C), 128.1, 127.8, 126.8 (2C), 116.0, 81.0, 62.0, 41.6, 27.9 (3C), 8.9; UPLC (Acquity BEH $\mathrm{C} 18, \mathrm{MeCN} / \mathrm{H}_{2} \mathrm{O} / \mathrm{TFA}=5 / 95 / 0.05 \rightarrow 95 / 5 / 0.05$, flow rate $\left.=1.0 \mathrm{~mL} / \mathrm{min}, \lambda=220 \mathrm{~nm}\right) \mathrm{tR}=1.33$ min; HRMS (ESI) m/z: [M+H] $]^{+}$calcd for $\mathrm{C}_{17} \mathrm{H}_{23} \mathrm{~N}_{2} \mathrm{O}_{2} 287.1760$; found 287.1743.

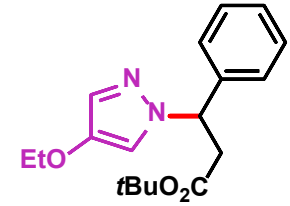

Substrate 8b: The standard procedure was followed with 4-ethoxypyrazole (112 $\mathrm{mg}, 1.0 \mathrm{mmol})$ and tert-butyl cinnamate $(204 \mathrm{mg}, 1.0 \mathrm{mmol})$ to afford, after column chromatography $(10 \rightarrow 50 \%$ EtOAc/hexanes $), 8 b(270 \mathrm{mg}, 0.9$ mmol, 85\%) as a colorless oil.

${ }^{1} \mathrm{H}$ NMR (600 MHz, $\left.\mathrm{CDCl}_{3}\right) \delta 7.33$ - 7.30 (m, 2H), 7.29 - 7.22 (m, 4H), 7.05 (br s, 1H), 5.62 (dd, $J=9.2,6.3 \mathrm{~Hz}, 1 \mathrm{H}), 3.87$ (qd, $J=7.0,1.4 \mathrm{~Hz}, 2 \mathrm{H}), 3.42$ (dd, $J=15.9,9.2 \mathrm{~Hz}, 1 \mathrm{H}), 2.99$ (dd, $J=15.9$, $6.3 \mathrm{~Hz}, 1 \mathrm{H}), 1.34(\mathrm{~s}, 9 \mathrm{H}), 1.32(\mathrm{t}, J=7.0 \mathrm{~Hz}, 3 \mathrm{H}) ;{ }^{13} \mathrm{C}\left\{{ }^{1} \mathrm{H}\right\} \mathrm{NMR}\left(151 \mathrm{MHz}, \mathrm{CDCl}_{3}\right) \delta 169.6,145.8$, 139.9, 128.8 (2C), 128.2, 127.3, 126.7 (2C), 114.4, 81.2, 67.3, 62.6, 41.4, 28.0 (3C), 15.0; UPLC (Acquity BEH C18, $\mathrm{MeCN} / \mathrm{H}_{2} \mathrm{O} / \mathrm{TFA}=5 / 95 / 0.05 \rightarrow 95 / 5 / 0.05$, flow rate $=1.0 \mathrm{~mL} / \mathrm{min}, \lambda=220$ $\mathrm{nm}) \mathrm{tR}=1.34 \mathrm{~min}$; HRMS (ESI) m/z: $[\mathrm{M}+\mathrm{H}]^{+}$calcd for $\mathrm{C}_{18} \mathrm{H}_{25} \mathrm{~N}_{2} \mathrm{O}_{3} 317.1865$; found 317.1871 .

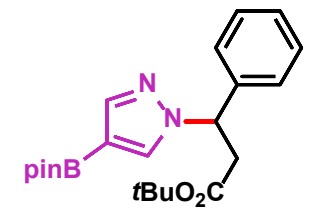

Substraet 8c: In a departure from the standard procedure, 4-(4,4,5,5tetramethyl-1,3,2-dioxaborolan-2-yl)-1H-pyrazole (388 mg, $2.0 \mathrm{mmol}, 2.0$ equiv) and tert-butyl cinnamate (204 mg, $1.0 \mathrm{mmol}, 1.0$ equiv) were combined in $\mathrm{MeCN}(1.0 \mathrm{~mL})$. Potassium tert-butoxide $(112 \mathrm{mg}, 1.0 \mathrm{mmol})$ was added and the reaction mixture was heated at $80{ }^{\circ} \mathrm{C}$ for $30 \mathrm{~min}, 60{ }^{\circ} \mathrm{C}$ for $1.5 \mathrm{~h}$, and was kept at room temperature for $16 \mathrm{~h}$. The reaction mixture was purified by column chromatography ( $5 \rightarrow 25 \%$ EtOAc/hexanes) to afford $\mathbf{8 c}(220 \mathrm{mg}, 0.6 \mathrm{mmol}, 55 \%)$ as a white solid, which contained a small 
amount (ca. 5\%) of proto-deborylated material (i.e., 6) that was inseparable from the desired product.

${ }^{1} \mathrm{H}$ NMR $\left(500 \mathrm{MHz}, \mathrm{CDCl}_{3}\right) \delta 7.80(\mathrm{~s}, 1 \mathrm{H}), 7.70(\mathrm{~s}, 1 \mathrm{H}), 7.32$ - $7.27(\mathrm{~m}, 5 \mathrm{H}), 5.76(\mathrm{dd}, J=8.8$, $6.6 \mathrm{~Hz}, 1 \mathrm{H}), 3.46$ (dd, $J=15.9,8.9 \mathrm{~Hz}, 1 \mathrm{H}), 3.04$ (dd, $J=15.9,6.6 \mathrm{~Hz}, 1 \mathrm{H}), 1.31(\mathrm{~s}, 9 \mathrm{H}), 1.28$ (s, $12 \mathrm{H}) ;{ }^{13} \mathrm{C}\left\{{ }^{1} \mathrm{H}\right\} \mathrm{NMR}\left(126 \mathrm{MHz}, \mathrm{CDCl}_{3}\right) \delta 169.3,145.4,139.3,136.0,128.9$ (2C), 128.3, 127.0 (2C), $\sim 106.7,{ }^{*} 83.3(2 \mathrm{C}), 81.3,62.3,41.6,27.9$ (3C), 24.9 (2C), $24.8(2 \mathrm{C}),{ }^{*}$ This peak corresponds to the carbon attached to boron and was not observed in the ${ }^{13} \mathrm{C} N M R$ experiment. It could be observed through an HMBC experiment, though its position is necessarily approximate; UPLC (Acquity BEH C18, $\mathrm{MeCN} / \mathrm{H}_{2} \mathrm{O} / \mathrm{NH}_{4} \mathrm{OH}=5 / 95 / 10 \mathrm{mM} \rightarrow$ 95/5/10 mM, flow rate $=1.0$ $\mathrm{mL} / \mathrm{min}, \lambda=220 \mathrm{~nm}$ ) $\mathrm{tR}=1.49 \mathrm{~min}$; HRMS (ESI) $\mathrm{m} / \mathrm{z}$ : $[\mathrm{M}+\mathrm{H}]^{+}$calc' for $\mathrm{C}_{22} \mathrm{H}_{32} \mathrm{~N}_{2} \mathrm{O}_{4}{ }^{11} \mathrm{~B}$ 399.2455; found 399.2450 .

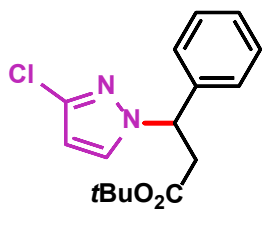

Substrate 8d: The standard procedure was followed with 3-chloropyrazole (103 mg, $1.0 \mathrm{mmol}$ ) and tert-butyl cinnamate (204 mg, $1.0 \mathrm{mmol})$ to afford, after column chromatography $(10 \rightarrow 50 \%$ EtOAc/hexanes $), 8 d$ (230 mg, 0.8 mmol, 75\%), a colorless liquid, as a single regioisomer (N-1:N-2 $>20: 1$ as judged by ${ }^{1} \mathrm{H}$ NMR of the crude reaction mixture).

${ }^{1} \mathrm{H}$ NMR $\left(500 \mathrm{MHz}, \mathrm{CDCl}_{3}\right) \delta 7.35$ - $7.28(\mathrm{~m}, 6 \mathrm{H}), 6.14$ (d, $\left.J=2.4 \mathrm{~Hz}, 1 \mathrm{H}\right), 5.65$ (dd, $J=9.1,6.1$ $\mathrm{Hz}, 1 \mathrm{H}), 3.45(\mathrm{dd}, J=16.0,9.1 \mathrm{~Hz}, 1 \mathrm{H}), 3.00(\mathrm{dd}, J=16.0,6.1 \mathrm{~Hz}, 1 \mathrm{H}), 1.34(\mathrm{~s}, 9 \mathrm{H}) ;{ }^{13} \mathrm{C}\left\{{ }^{1} \mathrm{H}\right\}$ NMR (126 MHz, $\left.\mathrm{CDCl}_{3}\right) \delta 169.4,139.3,138.9,131.1,129.0$ (2C), 128.6, 127.0 (2C), 105.1, 81.5, 63.1, 41.5, 28.0 (3C); UPLC (Acquity BEH C18, $\mathrm{MeCN} / \mathrm{H}_{2} \mathrm{O} / \mathrm{TFA}=5 / 95 / 0.05 \rightarrow$ 95/5/0.05, flow rate $=1.0 \mathrm{~mL} / \mathrm{min}, \lambda=220 \mathrm{~nm}$ ) $\mathrm{tR}=1.40 \mathrm{~min} ; \mathrm{HRMS}(\mathrm{ESI}) \mathrm{m} / \mathrm{z}:[\mathrm{M}+\mathrm{H}]^{+}$calcd for $\mathrm{C}_{16} \mathrm{H}_{20} \mathrm{~N}_{2} \mathrm{O}_{2} \mathrm{Cl} 307.1213$; found 307.1206.

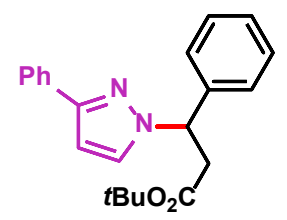

Substrate 8e: The standard procedure was followed with 3-phenypyrazole (144 mg, $1.0 \mathrm{mmol}$ ) and tert-butyl cinnamate (204 mg, $1.0 \mathrm{mmol})$ to afford, after column chromatography $(10 \rightarrow 50 \%$ EtOAc/hexanes), 8e (276 mg, 0.8 mmol, 79\%), a white solid, as a single regioisomer (N-1:N-2 $>20: 1$ as judged by ${ }^{1} \mathrm{H}$ NMR of the crude reaction mixture).

${ }^{1} \mathrm{H}$ NMR (500 MHz, $\left.\mathrm{CDCl}_{3}\right) \delta 7.91$ - 7.87 (m, 2H), 7.45 - $7.40(\mathrm{~m}, 3 \mathrm{H}), 7.40$ - $7.29(\mathrm{~m}, 6 \mathrm{H}), 6.58$ $(\mathrm{d}, J=2.3,1 \mathrm{H}), 5.82(\mathrm{dd}, J=9.0,6.6 \mathrm{~Hz}, 1 \mathrm{H}), 3.61(\mathrm{dd}, J=15.9,9.0 \mathrm{~Hz}, 1 \mathrm{H}), 3.11(\mathrm{dd}, J=15.9,6.4$ 
$\mathrm{Hz}, 1 \mathrm{H}), 1.37$ (s, 9H); ${ }^{13} \mathrm{C}\left\{{ }^{1} \mathrm{H}\right\}$ NMR (126 MHz, $\left.\mathrm{CDCl}_{3}\right) \delta 169.6,151.1,139.9,133.9,130.3$, 128.8 (2C), 128.6 (2C), 128.2, 127.5, 127.0 (2C), 125.7 (2C), 102.9, 81.1, 62.5, 42.0, 28.0 (3C); UPLC (Acquity BEH C18, MeCN/ $\mathrm{H}_{2} \mathrm{O} / \mathrm{TFA}=5 / 95 / 0.05 \rightarrow 95 / 5 / 0.05$, flow rate $=1.0 \mathrm{~mL} / \mathrm{min}$, $\lambda=220 \mathrm{~nm}$ ) $\mathrm{tR}=1.51 \mathrm{~min}$; HRMS (ESI) $\mathrm{m} / \mathrm{z}$ : $[\mathrm{M}+\mathrm{H}]^{+}$calcd for $\mathrm{C}_{22} \mathrm{H}_{25} \mathrm{~N}_{2} \mathrm{O}_{2} 349.1916$; found 349.1922 .

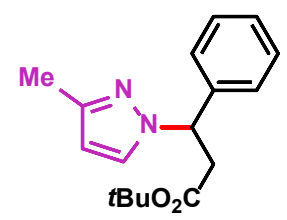

Substrate 8f The standard procedure was followed with 3-methylpyrazole (82 $\mathrm{mg}, 1.0 \mathrm{mmol}$ ) and tert-butyl cinnamate $(204 \mathrm{mg}, 1.0 \mathrm{mmol})$ to afford, after column chromatography $(10 \rightarrow 50 \%$ EtOAc/hexanes $), 8$ as a mixture of regioisomers (220 mg, $0.8 \mathrm{mmol}, 77 \%, 3.3: 1 \mathrm{~N}-1: \mathrm{N}-2)$ as a colorless oil. An analytically pure sample of each isomer could be isolated by careful preparatory HPLC (XBridge $\mathrm{C} 18, \mathrm{MeCN} / \mathrm{H}_{2} \mathrm{O} / \mathrm{NH}_{4} \mathrm{OAc}=32 / 68 / 10 \mathrm{mM} \rightarrow 72 / 28 / 10 \mathrm{mM}$, flow rate $=20.0 \mathrm{~mL} / \mathrm{min}, \lambda=220$ $\mathrm{nm}) \mathrm{tR}=16.7 \mathrm{~min}(\mathbf{8 f}), \mathrm{tR}=18.2 \mathrm{~min}(\mathbf{S I}-13)$ :

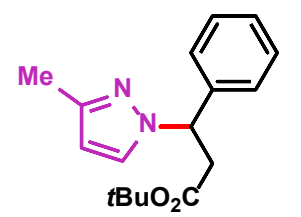

Major Isomer (8f):

${ }^{1} \mathrm{H}$ NMR (600 MHz, $\left.\mathrm{CDCl}_{3}\right) \delta 7.33$ (br d, $J=7.3 \mathrm{~Hz}, 2 \mathrm{H}$ ), $7.31-7.27$ (m, 4H), $6.01(\mathrm{~d}, J=2.0 \mathrm{~Hz}, 1 \mathrm{H}), 5.71(\mathrm{dd}, J=8.8,6.8 \mathrm{~Hz}, 1 \mathrm{H}), 3.42(\mathrm{dd}, J=15.7,8.8 \mathrm{~Hz}$, 1H), 3.05 (dd, $J=15.7,6.8 \mathrm{~Hz}, 1 \mathrm{H}), 2.29$ (s, 3H), 1.35 (s, 9H); ${ }^{13} \mathrm{C}\left\{{ }^{1} \mathrm{H}\right\} \mathrm{NMR}$ $\left(151 \mathrm{MHz}, \mathrm{CDCl}_{3}\right) \delta$ 169.6, 148.4, 139.9, 129.5, 128.8 (2C), 128.1, 126.9 (2C), 105.2, 81.1, 62.0, 41.9, 27.9 (3C), 13.8; UPLC (Acquity BEH C18, $\mathrm{MeCN} / \mathrm{H}_{2} \mathrm{O} / \mathrm{TFA}=5 / 95 / 0.05 \rightarrow$ 95/5/0.05, flow rate $=1.0 \mathrm{~mL} / \mathrm{min}, \lambda=220 \mathrm{~nm}$ ) $\mathrm{tR}=1.31 \mathrm{~min} ; \mathrm{HRMS}(\mathrm{ESI}) \mathrm{m} / \mathrm{z}[\mathrm{M}+\mathrm{H}]^{+}$calcd for $\mathrm{C}_{17} \mathrm{H}_{23} \mathrm{~N}_{2} \mathrm{O}_{2} 287.1760$; found 287.1767 .

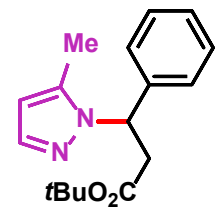

Minor Isomer (SI-13):

${ }^{1} \mathrm{H}$ NMR (600 MHz, $\left.\mathrm{CDCl}_{3}\right) \delta 7.44$ (s, 1H), 7.31 - 7.27 (m, 2H), 7.26 - 7.23 (m, 1H), 7.22 - 7.19 (m, 2H), 6.00 (s, 1H), 5.66 (dd, $J=9.9,5.3 \mathrm{~Hz}, 1 \mathrm{H}), 3.57$ (dd, $J=16.2,9.9 \mathrm{~Hz}, 1 \mathrm{H}), 3.00(\mathrm{dd}, J=16.2,5.3 \mathrm{~Hz}, 1 \mathrm{H}), 2.23$ (s, 3H), 1.33 (s, 9H); ${ }^{13} \mathrm{C}\left\{{ }^{1} \mathrm{H}\right\}$ NMR (151 MHz, CDCl$) \delta 170.0,140.4,138.6,138.3,128.9(2 \mathrm{C}), 127.9$ (2C), 126.6, 105.5, 81.0, 58.5, 42.1, 28.0 (3C), 11.2; UPLC (Acquity BEH C18, MeCN/ $\mathrm{H}_{2} \mathrm{O} / \mathrm{TFA}=5 / 95 / 0.05$ $\rightarrow 95 / 5 / 0.05$, flow rate $=1.0 \mathrm{~mL} / \mathrm{min}, \lambda=220 \mathrm{~nm}) \mathrm{tR}=1.35 \mathrm{~min} ; \mathrm{HRMS}(\mathrm{ESI}) \mathrm{m} / \mathrm{z}:[\mathrm{M}+\mathrm{H}]^{+}$ calcd for $\mathrm{C}_{17} \mathrm{H}_{23} \mathrm{~N}_{2} \mathrm{O}_{2} 287.1760$; found 287.1766. 


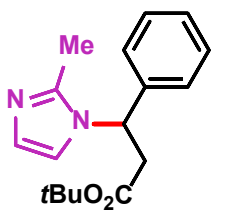

Substrate 8g: The standard procedure was followed with 2-methylimidazole (82 $\mathrm{mg}, 1.0 \mathrm{mmol})$ and tert-butyl cinnamate $(204 \mathrm{mg}, 1.0 \mathrm{mmol})$ to afford, after column chromatography $(10 \rightarrow 50 \%$ EtOAc/hexanes $), 8 g(190 \mathrm{mg}, 0.7 \mathrm{mmol}, 66 \%)$ as a colorless oil.

${ }^{1} \mathrm{H}$ NMR $\left(500 \mathrm{MHz}, \mathrm{CDCl}_{3}\right) \delta 7.31(\mathrm{dd}, J=8.2,7.4 \mathrm{~Hz}, 2 \mathrm{H}), 7.27$ (t, J=7.4 Hz, 1H), 7.09 (d, J=8.2 Hz, 2H), 6.97 (s, 1H), 6.95 (s, 1H), 5.62 (dd, $J=9.5,6.3 \mathrm{~Hz}, 1 \mathrm{H}), 3.08$ (dd, $J=15.9,6.3 \mathrm{~Hz}, 1 \mathrm{H})$, $3.03(\mathrm{dd}, J=15.9,9.5 \mathrm{~Hz}, 1 \mathrm{H}), 2.35(\mathrm{~s}, 3 \mathrm{H}), 1.32(\mathrm{~s}, 9 \mathrm{H}) ;{ }^{13} \mathrm{C}\left\{{ }^{1} \mathrm{H}\right\} \mathrm{NMR}\left(126 \mathrm{MHz}, \mathrm{CDCl}_{3}\right) \delta$ 168.9, 145.2, 139.4, 129.1 (2C), 128.2, 127.7, 126.2 (2C), 116.3, 81.9, 56.4, 42.5, 27.9 (3C), 13.5; UPLC (Acquity BEH C18, $\mathrm{MeCN} / \mathrm{H}_{2} \mathrm{O} / \mathrm{TFA}=5 / 95 / 0.05 \rightarrow 95 / 5 / 0.05$, flow rate $=1.0 \mathrm{~mL} / \mathrm{min}, \lambda$ $=220 \mathrm{~nm}) \mathrm{tR}=0.93 \mathrm{~min} ; \mathrm{HRMS}(\mathrm{ESI}) \mathrm{m} / \mathrm{z}:[\mathrm{M}+\mathrm{H}]^{+}$calcd for $\mathrm{C}_{17} \mathrm{H}_{23} \mathrm{~N}_{2} \mathrm{O}_{2}$ 287.1760; found 287.1751 .

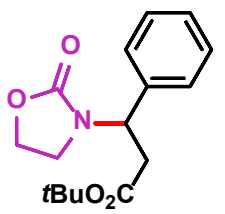

Substrate 8h: The standard procedure was followed with oxazolidin-2-one (87 mg, $1.0 \mathrm{mmol})$ and tert-butyl cinnamate $(204 \mathrm{mg}, 1.0 \mathrm{mmol})$ to afford, after column chromatography $(10 \rightarrow 50 \%$ EtOAc/hexanes), $8 \mathbf{h}(210 \mathrm{mg}, 0.7 \mathrm{mmol}, 72 \%)$ as a colorless oil.

${ }^{1} \mathrm{H}$ NMR (500 MHz, $\left.\mathrm{CDCl}_{3}\right) \delta 7.37$ - 7.27 (m, 5H), 5.40 (dd, J=9.6, 6.9 Hz, 1H), 4.25 (ddd, J=9.5, 8.0, 7.9 Hz, 1H), 4.21 (ddd, $J=9.5,7.9,6.1 \mathrm{~Hz}, 1 \mathrm{H}), 3.56$ (br ddd, $J=9.1,8.0,6.1 \mathrm{~Hz}, 1 \mathrm{H}), 3.22$ (dt, $J=9.1,7.9 \mathrm{~Hz}, 1 \mathrm{H}), 2.95$ (dd, $J=14.5,9.6 \mathrm{~Hz}, 1 \mathrm{H}), 2.89$ (dd, $J=14.5,6.9 \mathrm{~Hz}, 1 \mathrm{H}), 1.38$ (s, 9H); ${ }^{13} \mathrm{C}\left\{{ }^{1} \mathrm{H}\right\} \mathrm{NMR}\left(126 \mathrm{MHz}, \mathrm{CDCl}_{3}\right) \delta 169.5,157.8,137.6,128.9$ (2C), 128.4, 127.3 (2C), 81.5, 62.0, 53.9, 40.8, 37.7, 27.9 (3C); UPLC (Acquity BEH C18, $\mathrm{MeCN} / \mathrm{H}_{2} \mathrm{O} / \mathrm{TFA}=5 / 95 / 0.05 \rightarrow$ 95/5/0.05, flow rate $=1.0 \mathrm{~mL} / \mathrm{min}, \lambda=220 \mathrm{~nm}) \mathrm{tR}=1.11 \mathrm{~min} ; \mathrm{HRMS}(\mathrm{ESI}) \mathrm{m} / \mathrm{z}:[\mathrm{M}+\mathrm{H}]^{+}$calcd for $\mathrm{C}_{16} \mathrm{H}_{22} \mathrm{~N}-$ $\mathrm{O}_{4} 292.1549$; found 292.1542.

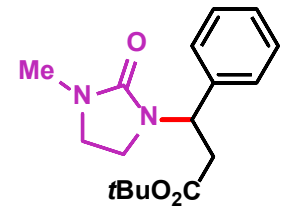

Substrate 8i: The standard procedure was followed with 1methylimidazolidin-2-one (100 mg, $1.0 \mathrm{mmol})$ and tert-butyl cinnamate (204 $\mathrm{mg}, 1.0 \mathrm{mmol})$ to afford, after column chromatography $(10 \rightarrow 50 \%$ EtOAc/hexanes), 8i (200 mg, $0.7 \mathrm{~mol}, 66 \%$ ) as a colorless oil. 
${ }^{1} \mathrm{H}$ NMR (600 MHz, $\left.\mathrm{CDCl}_{3}\right) \delta 7.34-7.24(\mathrm{~m}, 5 \mathrm{H}), 5.48(\mathrm{t}, J=8.2 \mathrm{~Hz}, 1 \mathrm{H}), 3.31(\mathrm{td}, J=8.3,5.7 \mathrm{~Hz}$, $1 \mathrm{H}), 3.20$ (q, $J=8.3 \mathrm{~Hz}, 1 \mathrm{H}), 3.18$ (td, $J=8.3,5.7 \mathrm{~Hz}, 1 \mathrm{H}), 2.95$ (q, $J=8.3 \mathrm{~Hz}, 1 \mathrm{H}), 2.88$ (d, $J=8.2$ $\mathrm{Hz}, 2 \mathrm{H}), 2.76(\mathrm{~s}, 3 \mathrm{H}), 1.35(\mathrm{~s}, 9 \mathrm{H}) ;{ }^{13} \mathrm{C}\left\{{ }^{1} \mathrm{H}\right\} \operatorname{NMR}\left(151 \mathrm{MHz}, \mathrm{CDCl}_{3}\right) \delta 170.1,160.8,138.7,128.6$ (2C), 127.8, 127.6 (2C), 81.1, 53.1, 45.3, 38.3, 37.9, 31.5, 28.0 (3C); UPLC (Acquity BEH C18, $\mathrm{MeCN} / \mathrm{H}_{2} \mathrm{O} / \mathrm{TFA}=5 / 95 / 0.05 \rightarrow 95 / 5 / 0.05$, flow rate $\left.=1.0 \mathrm{~mL} / \mathrm{min}, \lambda=220 \mathrm{~nm}\right) \mathrm{tR}=1.17 \mathrm{~min}$; HRMS (ESI) m/z: [M+H] $]^{+}$calcd for $\mathrm{C}_{17} \mathrm{H}_{25} \mathrm{~N}_{2} \mathrm{O}_{3} 305.1865$; found 305.1860 .

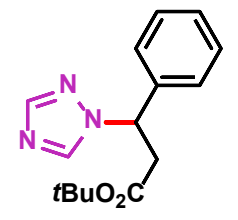

Substrate 8j: In a departure from the standard procedure, 1,2,4-triazole (138 mg, $2.0 \mathrm{mmol}, 2.0$ equiv), tert-butyl cinnamate (204 mg, $1.0 \mathrm{mmol}, 1.0$ equiv) and potassium tert-butoxide $(112 \mathrm{mg}, 1.0 \mathrm{mmol}, 1.0$ equiv) were combined and dissolved in $\mathrm{MeCN}(0.5 \mathrm{~mL})$. The reaction mixture was heated at $80^{\circ} \mathrm{C}$ for $44 \mathrm{~h}$, $60{ }^{\circ} \mathrm{C}$ for $48 \mathrm{~h}$, and then was kept at room temperature for $24 \mathrm{~h}$. The reaction mixture was directly purified by column chromatography ( $10 \rightarrow 50 \%$ EtOAc/hexanes) to afford $8 \mathbf{j}$ ( $192 \mathrm{mg}, 0.7 \mathrm{mmol}$, $70 \%$, a thick oil that solidified upon standing, as a single isomer (N-1:N-4 $>20: 1$ as judged by ${ }^{1} \mathrm{H}$ NMR of the crude reaction mixture).

${ }^{1} \mathrm{H}$ NMR $\left(600 \mathrm{MHz}, \mathrm{CDCl}_{3}\right) \delta 8.08(\mathrm{~s}, 1 \mathrm{H}), 7.94(\mathrm{~s}, 1 \mathrm{H}), 7.38$ - $7.30(\mathrm{~m}, 5 \mathrm{H}), 5.82(\mathrm{dd}, J=9.4,5.8$ $\mathrm{Hz}, 1 \mathrm{H}), 3.46(\mathrm{dd}, J=16.2,9.4 \mathrm{~Hz}, 1 \mathrm{H}), 3.04(\mathrm{dd}, J=16.2,5.8 \mathrm{~Hz}, 1 \mathrm{H}), 1.33(\mathrm{~s}, 9 \mathrm{H}) ;{ }^{13} \mathrm{C}\left\{{ }^{1} \mathrm{H}\right\} \mathrm{NMR}$ $\left(151 \mathrm{MHz}, \mathrm{CDCl}_{3}\right) \delta$ 169.0, 152.0, 143.2, 138.0, 129.1 (2C), 128.9, $127.1(2 \mathrm{C}), 81.8,60.5,41.3$, 28.0 (3C); UPLC (Acquity BEH C18, $\mathrm{MeCN} / \mathrm{H}_{2} \mathrm{O} / \mathrm{TFA}=5 / 95 / 0.05 \rightarrow 95 / 5 / 0.05$, flow rate $=1.0$ $\mathrm{mL} / \mathrm{min}, \lambda=220 \mathrm{~nm}$ ) tR $=1.25 \mathrm{~min}$; HRMS (ESI) $\mathrm{m} / \mathrm{z}$ : $[\mathrm{M}+\mathrm{H}]^{+}$calc'd for $\mathrm{C}_{15} \mathrm{H}_{20} \mathrm{~N}_{3} \mathrm{O}_{2} 274.155$; found 274.1559 .

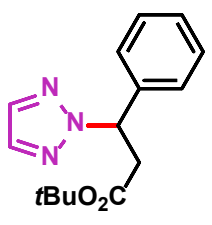

Substrate 8k: In a departure from the standard procedure, 1,2,3-triazole $(138 \mathrm{mg}$, $2.0 \mathrm{mmol}, 2.0$ equiv), tert-butyl cinnamate (204 mg, $1.0 \mathrm{mmol}, 1.0$ equiv) and potassium tert-butoxide (112 $\mathrm{mg}, 1.0 \mathrm{mmol}, 1.0$ equiv) were combined and dissolved in $\mathrm{MeCN}(0.5 \mathrm{~mL})$. The reaction mixture was heated at $80{ }^{\circ} \mathrm{C}$ for $44 \mathrm{~h}, 60$ ${ }^{\circ} \mathrm{C}$ for $48 \mathrm{~h}$, and then was kept at room temperature for $24 \mathrm{~h}$. The reaction mixture was directly purified by column chromatography $(10 \rightarrow 50 \%$ EtOAc/hexanes) to afford $\mathbf{8 k}(180 \mathrm{mg}, 0.7 \mathrm{mmol}$, $66 \%)$ as a colorless oil and SI-14 (31 mg, $0.1 \mathrm{mmol}, 11 \%$ ) an oil that crystallized upon standing. Overall, this represents a $76 \%$ yield $(211 \mathrm{mg}, 0.8 \mathrm{mmol})$ of Michael addition products afforded in a 6:1 ratio. 


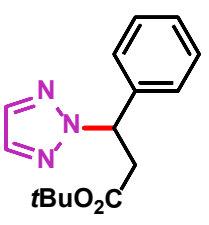

Major isomer (8k):

${ }^{1} \mathrm{H}$ NMR $\left(600 \mathrm{MHz}, \mathrm{CDCl}_{3}\right) \delta 7.60(\mathrm{~s}, 2 \mathrm{H}), 7.36-7.25(\mathrm{~m}, 5 \mathrm{H}), 6.14(\mathrm{dd}, J=9.5$, $6.1 \mathrm{~Hz}, 1 \mathrm{H}), 3.57$ (dd, $J=16.2,9.5 \mathrm{~Hz}, 1 \mathrm{H}), 3.12$ (dd, $J=16.2,6.1 \mathrm{~Hz}, 1 \mathrm{H}), 1.32$ (s, $9 \mathrm{H}) ;{ }^{13} \mathrm{C}\left\{{ }^{1} \mathrm{H}\right\}$ NMR $\left(151 \mathrm{MHz}, \mathrm{CDCl}_{3}\right) \delta 168.9,138.6,134.2(2 \mathrm{C}), 128.8(2 \mathrm{C})$, 128.5, 126.8 (2C), 81.3, 65.2, 41.5, 27.9; UPLC (Acquity BEH C18, $\mathrm{MeCN} / \mathrm{H}_{2} \mathrm{O} / \mathrm{TFA}=5 / 95 / 0.05$ $\rightarrow 95 / 5 / 0.05$, flow rate $=1.0 \mathrm{~mL} / \mathrm{min}, \lambda=220 \mathrm{~nm}$ ) $\mathrm{tR}=1.25 \mathrm{~min}$; HRMS $(\mathrm{ESI}) \mathrm{m} / \mathrm{z}:[\mathrm{M}+\mathrm{H}]^{+}$calcd for $\mathrm{C}_{15} \mathrm{H}_{20} \mathrm{~N}_{3} \mathrm{O}_{2} 274.1556$; found 274.1544.

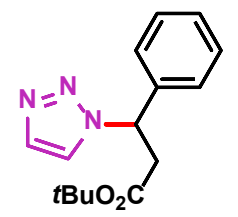

Minor isomer (SI-14):

${ }^{1} \mathrm{H}$ NMR (600 MHz, $\left.\mathrm{CDCl}_{3}\right) \delta 7.70(\mathrm{~s}, 1 \mathrm{H}), 7.54(\mathrm{~s}, 1 \mathrm{H}), 7.42$ - 7.30 (m, 5H), 6.04 (dd, $J=9.3,6.2 \mathrm{~Hz}, 1 \mathrm{H}), 3.64$ (dd, $J=16.2,9.3 \mathrm{~Hz}, 1 \mathrm{H}), 3.16$ (dd, $J=16.2,6.2 \mathrm{~Hz}$, 1H), $1.36(\mathrm{~s}, 9 \mathrm{H}) ;{ }^{13} \mathrm{C}\left\{{ }^{1} \mathrm{H}\right\}$ NMR (151 MHz, $\left.\mathrm{CDCl}_{3}\right) \delta 168.8,138.1,133.8,129.1$

(2C), 128.9, 126.9 (2C), 123.5, 81.8, 61.4, 41.6, 27.9 (3C); UPLC (Acquity BEH C18, $\mathrm{MeCN} / \mathrm{H}_{2} \mathrm{O} / \mathrm{TFA}=5 / 95 / 0.05 \rightarrow 95 / 5 / 0.05$, flow rate $\left.=1.0 \mathrm{~mL} / \mathrm{min}, \lambda=220 \mathrm{~nm}\right) \mathrm{tR}=1.10 \mathrm{~min}$; HRMS (ESI) m/z: [M+H] $]^{+}$calcd for $\mathrm{C}_{15} \mathrm{H}_{20} \mathrm{~N}_{3} \mathrm{O}_{2} 274.1556$; found 274.1550 .

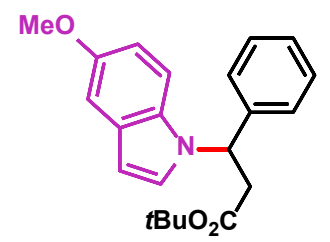

Substrate 81: The standard procedure was followed with 5-methoxyindole (147 mg, $1.0 \mathrm{mmol}$ ) and tert-butyl cinnamate (204 mg, $1.0 \mathrm{mmol})$ to afford, after column chromatography $(10 \rightarrow 50 \%$ EtOAc/hexanes), 81 ( $210 \mathrm{mg}, 0.6$ $\mathrm{mmol}, 60 \%)$ as a white solid.

${ }^{1} \mathrm{H}$ NMR (600 MHz, CDCL 3$) \delta 7.29$ (br t, $\left.J=7.1 \mathrm{~Hz}, 2 \mathrm{H}\right), 7.27-7.21$ (m, 3H), 7.18 (br d, $J=7.4$ Hz, 2H), 7.08 (d, $J=2.2 \mathrm{~Hz}, 1 \mathrm{H}), 6.83$ (dd, $J=8.9,2.2 \mathrm{~Hz}, 1 \mathrm{H}), 6.48$ (d, $J=3.1 \mathrm{~Hz}, 1 \mathrm{H}), 5.97$ (t, $J=7.8 \mathrm{~Hz}, 1 \mathrm{H}), 3.84(\mathrm{~s}, 3 \mathrm{H}), 3.21(\mathrm{~d}, J=7.8 \mathrm{~Hz}, 2 \mathrm{H}), 1.26(\mathrm{~s}, 9 \mathrm{H}) ;{ }^{13} \mathrm{C}\left\{{ }^{1} \mathrm{H}\right\}$ NMR $(151 \mathrm{MHz}$, $\left.\mathrm{CDCl}_{3}\right) \delta$ 169.4, 154.2, 140.3, 131.6, 129.1, 128.9 (2C), 128.0, $126.4(2 \mathrm{C}), 125.7,112.0,110.8$, 102.6, 101.8, 81.6, 56.6, 55.9, 42.0, 27.9 (3C); UPLC (Acquity BEH C18, MeCN/ $\mathrm{H}_{2} \mathrm{O} / \mathrm{TFA}=$ $5 / 95 / 0.05 \rightarrow 95 / 5 / 0.05$, flow rate $=1.0 \mathrm{~mL} / \mathrm{min}, \lambda=220 \mathrm{~nm}$ ) $\mathrm{tR}=1.49 \mathrm{~min}$; HRMS (ESI) calcd for $\mathrm{C}_{22} \mathrm{H}_{26} \mathrm{NO}_{3} 352.1913$; found 352.1922 . 


\section{Synthesis of MK-0429 (1):}

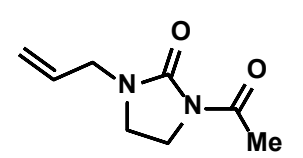

Intermediate SI-15: 1-acetylimidazolidin-2-one (10 g, $78 \mathrm{mmol}, 1.0$ equiv) was suspended in DMF (78 mL). NaHMDS solution (1.0 M in THF, $86 \mathrm{~mL}, 86$ mmol, 1.1 equiv) was addeed dropwise followed by allyl bromide $(9.5 \mathrm{~mL}, 109$ mmol, 1.4 equiv) as a neat liquid. The reaciton mixture was stirred at room temperature for $1 \mathrm{~h}$. The reaction mixture was quenched by addition of $\mathrm{HCl}$ solution $(1 \mathrm{M}, 50 \mathrm{~mL})$ and EtOAc was added $(50 \mathrm{~mL})$. The layers were separated and the aqueous layer was further extracted with EtOAc $(2 \times 50 \mathrm{~mL})$. The combined organic phases were washed with brine, dried over sodium sulfate, filtered, and concentrated. The crude residue (still containing a significant amount of DMF) was purified by column chromatography (70 $\rightarrow$ 100\% EtOAc/hexanes) to afford SI-15 (9.1 g, 54 mmol, 69\%) as a clear, colorless liquid.

${ }^{1} \mathrm{H}$ NMR $\left(500 \mathrm{MHz}, \mathrm{CDCl}_{3}\right) \delta 5.81$ - $5.71(\mathrm{~m}, 1 \mathrm{H}), 5.27$ - $5.24(\mathrm{~m}, 1 \mathrm{H}), 5.24-5.21(\mathrm{~m}, 1 \mathrm{H}), 3.89$ - $3.86(\mathrm{~m}, 2 \mathrm{H}), 3.85-3.80(\mathrm{~m}, 2 \mathrm{H}), 3.40-3.35(\mathrm{~m}, 2 \mathrm{H}), 2.50(\mathrm{~s}, 3 \mathrm{H}) ;{ }^{13} \mathrm{C}\left\{{ }^{1} \mathrm{H}\right\} \mathrm{NMR}(126 \mathrm{MHz}$, $\left.\mathrm{CDCl}_{3}\right) \delta 170.9,154.7,132.0,118.9,46.6,40.6,39.6,23.4$; UPLC (Acquity BEH C18, $\mathrm{MeCN} / \mathrm{H}_{2} \mathrm{O} / \mathrm{TFA}=5 / 95 / 0.05 \rightarrow 95 / 5 / 0.05$, flow rate $\left.=1.0 \mathrm{~mL} / \mathrm{min}, \lambda=220 \mathrm{~nm}\right) \mathrm{tR}=0.79 \mathrm{~min}$; HRMS (ESI) m/z: [M+H] $]^{+}$calcd for $\mathrm{C}_{8} \mathrm{H}_{13} \mathrm{~N}_{2} \mathrm{O}_{2}$ 169.0977; found 169.0985 .

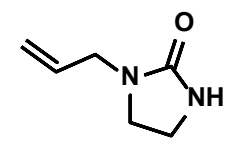

N-Allylimidazolidin-2-one (10): SI-15 (22 g, $131 \mathrm{mmol}, 1.0$ equiv) was dissolved in $\mathrm{MeOH}(262 \mathrm{~mL})$ and sodium hydroxide solution (1.0 M, $196 \mathrm{~mL}, 196 \mathrm{mmol}, 1.5$ equiv) was added. The reaction mixture was stirred at room temperature for $1 \mathrm{~h}$. The reaction mixture was quenched with $\mathrm{HCl}$ solution $(1.0 \mathrm{M}, 100 \mathrm{~mL})$ until $\mathrm{pH} 6$ was reached and $\mathrm{MeOH}$ was removed by rotary evaporation. The remaining aqueous phase was extracted with 3:1 DCM:MeOH $(5 \times 100 \mathrm{~mL})$. The combined organic phases were dried over sodium sulfate, filtered, and concentrated to afford $\mathbf{1 0}(14.2 \mathrm{~g}, 113 \mathrm{mmol}, 86 \%)$, a thick oil, which could be used directly in the next step, without additional purification.

${ }^{1} \mathrm{H}$ NMR (600 MHz, $\left.\mathrm{CDCl}_{3}\right) \delta 5.73$ (ddt, $\left.J=16.8,10.3,6.0 \mathrm{~Hz}, 1 \mathrm{H}\right), 5.50$ (br s, 1H), 5.18 (dd, $J=16.9,0.9 \mathrm{~Hz}, 1 \mathrm{H}), 5.14$ (dd, $J=10.3,0.9 \mathrm{~Hz}, 1 \mathrm{H}), 3.75$ (br d, $J=6.0 \mathrm{~Hz}, 2 \mathrm{H}), 3.42$ - 3.32 (m, 4H); ${ }^{13} \mathrm{C}\left\{{ }^{1} \mathrm{H}\right\} \mathrm{NMR}\left(151 \mathrm{MHz}, \mathrm{CDCl}_{3}\right) \delta 163.0,133.5,117.5,46.4,44.8,38.2$; UPLC (Acquity BEH $\mathrm{C} 18, \mathrm{MeCN} / \mathrm{H}_{2} \mathrm{O} / \mathrm{TFA}=5 / 95 / 0.05 \rightarrow 95 / 5 / 0.05$, flow rate $\left.=1.0 \mathrm{~mL} / \mathrm{min}, \lambda=220 \mathrm{~nm}\right) \mathrm{tR}=0.66$ min; HRMS (ESI) m/z: [M+H] $]^{+}$calcd for $\mathrm{C}_{6} \mathrm{H}_{11} \mathrm{~N}_{2} \mathrm{O}$ 127.0871, found: 127.0868 . 


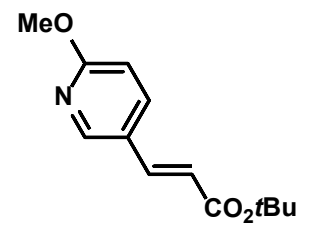

Acrylate 11: The standard procedure for acrylate synthesis was followed with 6-methoxynicotinaldehyde ( $8.0 \mathrm{~g}, 58 \mathrm{mmol}, 1.0$ equiv) to afford, after column chromatography $(10 \rightarrow 25 \%$ EtOAc/hexanes), $11(11 \mathrm{~g}, 47 \mathrm{mmol}, 80 \%)$ as a white solid $(E: Z>20: 1)$.

${ }^{1} \mathrm{H}$ NMR $\left(600 \mathrm{MHz}, \mathrm{CDCl}_{3}\right) \delta 8.23(\mathrm{~d}, J=2.0 \mathrm{~Hz}, 1 \mathrm{H}), 7.73(\mathrm{dd}, J=8.7,2.0 \mathrm{~Hz}, 1 \mathrm{H}), 7.51(\mathrm{~d}, J=16.0$ $\mathrm{Hz}, 1 \mathrm{H}), 6.74(\mathrm{~d}, J=8.7 \mathrm{~Hz}, 1 \mathrm{H}), 6.25(\mathrm{~d}, J=16.0 \mathrm{~Hz}, 1 \mathrm{H}), 3.95(\mathrm{~s}, 3 \mathrm{H}), 1.52(\mathrm{~s}, 9 \mathrm{H}) ;{ }^{13} \mathrm{C}\left\{{ }^{1} \mathrm{H}\right\} \mathrm{NMR}$ $\left(126 \mathrm{MHz}, \mathrm{CDCl}_{3}\right) \delta 166.2,165.2,148.2,140.0,136.4,124.1,119.3,111.6,80.7,53.9,28.3(\mathrm{~s}$, 3C); UPLC (Acquity BEH C18, $\mathrm{MeCN} / \mathrm{H}_{2} \mathrm{O} / \mathrm{TFA}=5 / 95 / 0.05 \rightarrow 95 / 5 / 0.05$, flow rate $=1.0$ $\mathrm{mL} / \mathrm{min}, \lambda=220 \mathrm{~nm}$ ) $\mathrm{tR}=1.31 \mathrm{~min}$; HRMS (ESI) calc'd for $\mathrm{C}_{13} \mathrm{H}_{18} \mathrm{NO}_{3} 236.1287$; found 236.1291.

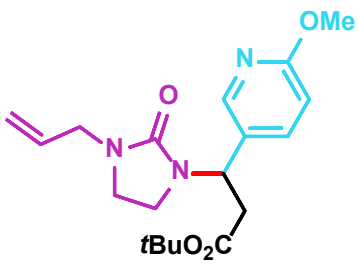

Michael Adduct 12: In a departure from the standard procedure for Michael adduct synthesis, 10 (14.2 g, $113 \mathrm{mmol}, 1.2$ equiv) was dissolved in $\mathrm{MeCN}(24 \mathrm{~mL})$ and potassium tert-butoxide $(2.6 \mathrm{~g}, 23 \mathrm{mmol}, 0.24$ equiv) was added. After $5 \mathrm{~min}, 11$ (22.4 g, $95 \mathrm{mmol}, 1.0$ equiv) was added. The reaction mixture was heated at $80{ }^{\circ} \mathrm{C}$ for $30 \mathrm{~min}, 60^{\circ} \mathrm{C}$ for $4 \mathrm{~h}$, and was then kept at room temperature for $70 \mathrm{~h}$. The reaction mixture was directly purified by column chromatography (20 $\rightarrow 100 \%$ EtOAc/hexanes) to afford $\mathbf{1 2}$ (15.8 g, $44 \mathrm{mmol}, 46 \%)$ as an off-white solid.

${ }^{1} \mathrm{H}$ NMR $\left(500 \mathrm{MHz}, \mathrm{CDCl}_{3}\right) \delta 8.07(\mathrm{~d}, J=2.3 \mathrm{~Hz}, 1 \mathrm{H}), 7.57(\mathrm{dd}, J=8.6,2.3 \mathrm{~Hz}, 1 \mathrm{H}), 6.70(\mathrm{~d}, J=8.6$ Hz, 1H), 5.71 (ddt, $J=17.2,9.0,6.2 \mathrm{~Hz}, 1 \mathrm{H}$ ), 5.41 (dd, $J=8.9,7.3 \mathrm{~Hz}, 1 \mathrm{H}$ ), 5.15 (br dt, $J=17.2,1.2$ $\mathrm{Hz}, 1 \mathrm{H}), 5.14$ (dt, $J=9.0,1.2 \mathrm{~Hz}, 1 \mathrm{H}), 3.91$ (s, $3 \mathrm{H}), 3.77$ (dd, $J=6.2,1.2 \mathrm{~Hz}, 2 \mathrm{H}), 3.32(\mathrm{td}, J=8.2$, $6.0 \mathrm{~Hz}, 1 \mathrm{H}), 3.20$ (q, $J=8.2 \mathrm{~Hz}, 1 \mathrm{H}), 3.16$ (td, $J=8.2,6.0 \mathrm{~Hz}, 1 \mathrm{H}), 2.99$ (q, $J=8.2 \mathrm{~Hz}, 1 \mathrm{H}), 2.89$ (dd, $J=14.4,8.9 \mathrm{~Hz}, 1 \mathrm{H}), 2.87(\mathrm{dd}, J=14.4,7.3 \mathrm{~Hz}, 1 \mathrm{H}), 1.37(\mathrm{~s}, 9 \mathrm{H}) ;{ }^{13} \mathrm{C}\left\{{ }^{1} \mathrm{H}\right\} \mathrm{NMR}\left(126 \mathrm{MHz}, \mathrm{CDCl}_{3}\right)$ $\delta$ 169.6, 163.7, 159.8, 145.4, 138.4, 133.3, 126.9, 117.6, 110.8, 81.1, 53.4, 50.8, 47.0, 42.3, 38.3, 37.7, 27.9 (3C); UPLC (Acquity BEH C18, $\mathrm{MeCN} / \mathrm{H}_{2} \mathrm{O} / \mathrm{TFA}=5 / 95 / 0.05 \rightarrow 95 / 5 / 0.05$, flow rate $=1.0 \mathrm{~mL} / \mathrm{min}, \lambda=220 \mathrm{~nm}$ ) tR $=1.15 \mathrm{~min}$; HRMS (ESI) calcd for $\mathrm{C}_{19} \mathrm{H}_{28} \mathrm{~N}_{3} \mathrm{O}_{4} 362.2080$; found 362.2070 . 


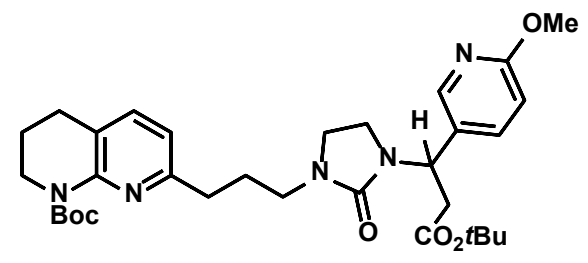

Protected MK-0429 14: 12 (12.0 g, 33 mmol, 1.05 equiv) was placed in a $1 \mathrm{~L}$ flask. The atmosphere was evacuated and replaced with nitrogen this process was performed 3 times. Separately, commercial 9-BBN solution (0.5 M in THF, 200 $\mathrm{mL}, 100 \mathrm{mmol}, 3.15$ equiv) was degassed by sparging through nitrogen. This solution was added directly to the solid $\mathbf{1 2}$ and the reaction mixture was stirred at room temperature for $1 \mathrm{~h}$. Potassium phosphate solution (2.0 M, $50 \mathrm{~mL}, 100 \mathrm{mmol}, 3.15$ equiv) was degassed by sparging through nitrogen and then was added to the reaction mixture. Separately, 13 (8.5 g, $32 \mathrm{mmol}, 1.0$ equiv) and SPhos Pd G1, MTBE adduct ( $0.43 \mathrm{~g}, 0.6 \mathrm{mmol}, 0.02$ equiv) were combined and dissolved in dioxane $(200 \mathrm{~mL})$. This solution was likewise degassed by sparging through nitrogen and was added to the reaction mixture. The combined biphasic solution was heated in an oil bath at $85^{\circ} \mathrm{C}$ for $1 \mathrm{~h}$. The reaction mixture was cooled to room temperature and was diluted with EtOAc $(300 \mathrm{~mL})$ and brine $(200 \mathrm{~mL})$. The layers were separated, and the organic phase was dried over sodium sulfate, filtered, and concentrated. The crude residue was purified by column chromatography ( $50 \rightarrow 100 \%$ EtOAc/hexanes) to afford $\mathbf{1 4}(16.3 \mathrm{~g}, 27 \mathrm{mmol}, 87 \%)$ as a white foam.

${ }^{1} \mathrm{H}$ NMR (600 MHz, $\left.\mathrm{CDCl}_{3}\right) \delta 8.05(\mathrm{~s}, 1 \mathrm{H}), 7.54(\mathrm{~d}, J=8.5 \mathrm{~Hz}, 1 \mathrm{H}), 7.21(\mathrm{~d}, J=7.5 \mathrm{~Hz}, 1 \mathrm{H}), 6.77$ (d, $J=7.5 \mathrm{~Hz}, 1 \mathrm{H}$ ), 6.68 (d, $J=8.5 \mathrm{~Hz}, 1 \mathrm{H}), 5.37$ (t, $J=8.2 \mathrm{~Hz}, 1 \mathrm{H}$ ), 3.88 (s, 3H), 3.69 (br t, $J=5.9$ $\mathrm{Hz}, 2 \mathrm{H}), 3.29$ - 3.15 (m, 5H), $2.94-2.88(\mathrm{~m}, 1 \mathrm{H}), 2.84$ (br d, $J=8.2 \mathrm{~Hz}, 2 \mathrm{H}), 2.70$ - 2.63 (m, 4H), 1.93 - 1.83 (m, 4H), 1.46 (s, 9H), $1.33(\mathrm{~s}, 9 \mathrm{H}) ;{ }^{13} \mathrm{C}\left\{{ }^{1} \mathrm{H}\right\} \mathrm{NMR}\left(151 \mathrm{MHz}, \mathrm{CDCl}_{3}\right) \delta$ 169.7, 163.7, 160.2, 157.5, 154.1, 151.1, 145.5, 138.6, 137.3, 127.1, 122.0, 118.5, 110.9, 81.2, 80.7, 53.5, 50.8, 44.9, 43.9, 42.7, 38.5, 37.8, 35.1, 28.4 (3C), 28.0 (3C), 27.4, 26.3, 23.3; UPLC (Acquity BEH C18, $\mathrm{MeCN} / \mathrm{H}_{2} \mathrm{O} / \mathrm{TFA}=5 / 95 / 0.05 \rightarrow 95 / 5 / 0.05$, flow rate $\left.=1.0 \mathrm{~mL} / \mathrm{min}, \lambda=220 \mathrm{~nm}\right) \mathrm{tR}=1.30 \mathrm{~min}$; HRMS (ESI) m/z: [M+H] $]^{+}$calcd for $\mathrm{C}_{32} \mathrm{H}_{46} \mathrm{~N}_{5} \mathrm{O}_{6}$ 596.3448; found 596.3444 .

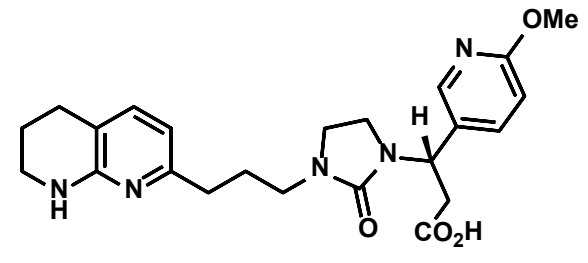

MK-0429 (1): 14 (14.9 g, 25 mmol, 1.0 equiv) was dissolved in DCM $(200 \mathrm{~mL})$ and TFA $(50 \mathrm{~mL})$. The reaction mixture was stirred at room temperature for $20 \mathrm{~h}$. The reaction mixture was azeotroped from $\mathrm{MeCN}$ and PhMe (1:1, $200 \mathrm{~mL})$ three times. The residue was taken up in $\mathrm{MeCN}(100 \mathrm{~mL})$ and saturated $\mathrm{NH}_{4} \mathrm{OH}$ solution was added until the solution had been neutralized ( $c a .5 \mathrm{~mL}$ ). This solution was concentrated and the crude material was purified by preparative chiral SFC (ChiralPak AD- $\mathrm{H}, \mathrm{MeOH} / \mathrm{MeCN} / \mathrm{CO}_{2} / \mathrm{NH}_{4} \mathrm{OH}=$ 
25/25/50/0.001, flow rate $=220 \mathrm{~mL} / \mathrm{min}, \lambda=220 \mathrm{~nm})$ to afford $\mathbf{1}(\mathrm{tR}=2.5 \mathrm{~min}, 4.2 \mathrm{~g}, 9.6 \mathrm{mmol}$, $38 \%$ ) and ent-1 (tR $=1.5 \mathrm{~min}, 4.3 \mathrm{~g}, 9.6 \mathrm{mmol}, 39 \%$ ), both of which were deemed to be $>99 \%$ ee by analytical chiral SFC (ChiralPak AD-H OD-H, $\mathrm{MeCN} / \mathrm{MeOH} / \mathrm{CO}_{2} / \mathrm{NH}_{4} \mathrm{OH}=$ $27.5 / 27.5 / 45 / 0.001$, flow rate $=3.0 \mathrm{~mL} / \mathrm{min}, \lambda=220-380 \mathrm{~nm}) \mathrm{tR}=1.7 \mathrm{~min}($ ent -1$), \mathrm{tR}=2.8 \mathrm{~min}$ (1). The assignment of the biologically active enantiomer was made unambiguously by analysis of a chiral SFC coinjection with authentically prepared MK-0429. ${ }^{6}$ Spectral data for 1 were in good agreement with the literature. ${ }^{6}$

${ }^{1} \mathrm{H}$ NMR $\left(500 \mathrm{MHz}, \mathrm{CD}_{3} \mathrm{OD}\right) \delta 8.09(\mathrm{~d}, J=2.4 \mathrm{~Hz}, 1 \mathrm{H}), 7.67$ (dd, $\left.J=8.6,2.4 \mathrm{~Hz}, 1 \mathrm{H}\right), 7.47$ (d, $J=7.2 \mathrm{~Hz}, 1 \mathrm{H}), 6.81$ (d, $J=8.6 \mathrm{~Hz}, 1 \mathrm{H}), 6.55(\mathrm{~d}, J=7.2 \mathrm{~Hz}, 1 \mathrm{H}), 5.49$ (dd, $J=12.1,2.9 \mathrm{~Hz}, 1 \mathrm{H}), 3.90$ (s, 3H), 3.66 (q, $J=9.3 \mathrm{~Hz}, 1 \mathrm{H}), 3.52$ (td, $J=9.3,3.4 \mathrm{~Hz}, 1 \mathrm{H}), 3.46$ (t, $J=5.5 \mathrm{~Hz}, 2 \mathrm{H}), 3.36$ (td, $J=13.4,3.6 \mathrm{~Hz}, 1 \mathrm{H}), 3.16$ (q, $J=9.3 \mathrm{~Hz}, 1 \mathrm{H}), 2.98$ (t, $J=12.3 \mathrm{~Hz}, 1 \mathrm{H}), 2.99(\mathrm{td}, J=9.3,3.4 \mathrm{~Hz}, 1 \mathrm{H})$, 2.82 - 2.75 (m, 4H), 2.66 (td, $J=13.4,4.4 \mathrm{~Hz}, 1 \mathrm{H}), 2.54$ (dd, $J=12.1,3.1 \mathrm{~Hz}, 1 \mathrm{H}), 2.14$ - 2.04 (m, 1H), $2.00-1.88(\mathrm{~m}, 2 \mathrm{H}), 1.88-1.79(\mathrm{~m}, 1 \mathrm{H}) ;{ }^{13} \mathrm{C}\left\{{ }^{1} \mathrm{H}\right\}$ NMR (126 MHz, CD $\left.{ }_{3} \mathrm{OD}\right) \delta$ 180.6, 165.1, 162.5, 153.3, 150.2, 146.6, 141.4, 139.7, 129.9, 119.5, 111.6, 110.7, 54.1, 53.0, 42.3, 41.6, 41.1, 38.8, 38.7, 29.3, 27.9, 26.6, 20.7; UPLC (Acquity BEH C18, MeCN/ $\mathrm{H}_{2} \mathrm{O} / \mathrm{TFA}=5 / 95 / 0.05 \rightarrow$ 95/5/0.05, flow rate $=1.0 \mathrm{~mL} / \mathrm{min}, \lambda=220 \mathrm{~nm}$ ) $\mathrm{tR}=0.91 \mathrm{~min}$; HRMS (ESI) $\mathrm{m} / \mathrm{z}:[\mathrm{M}+\mathrm{H}]^{+}$calcd for $\mathrm{C}_{23} \mathrm{H}_{30} \mathrm{~N}_{5} \mathrm{O}_{4} 440.2298$; found 440.2292 . 


\section{Chiral SFC results:}

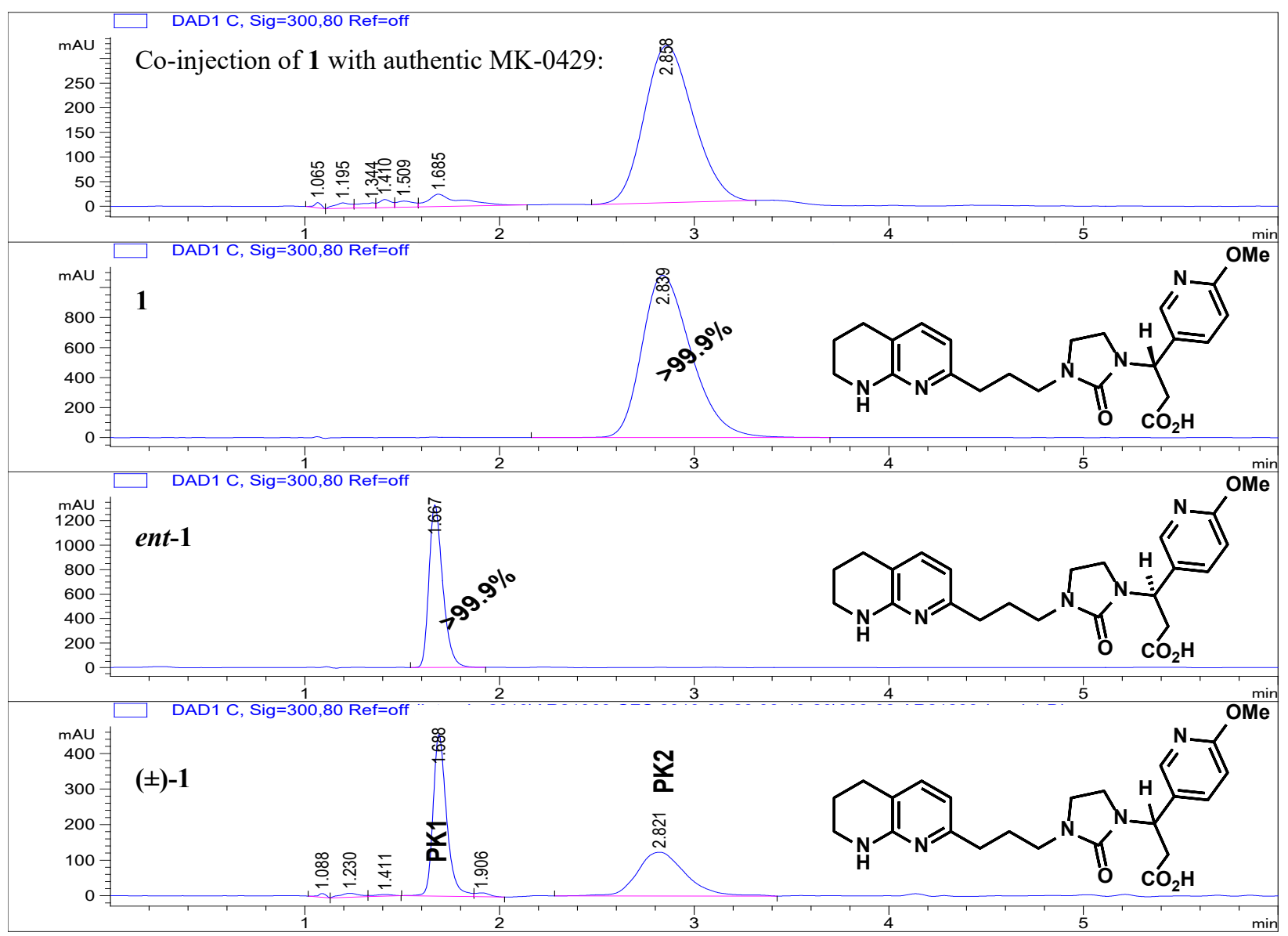




\section{References:}

(1) Davies, A. J.; Brands, K. M. J.; Cowden, C. J; Dolling, U.-H.; Lieberman, D. R. An efficient one-pot synthesis of annulated pyridines utilising a directed ortho-metallation/transmetallation approach. Tetrahedron Lett. 2004, 45, 1721.

(2) Wang, Z.; Feng, X.; Fang, W.; Tu, T. Efficient aqueous-phase Heck reaction catalyzed by a robust hyrophilic pyridine-bridged bisbenzimidazolylidene-palladium pincer complex. Synlett 2011, 951.

(3) Doi, H.; Sakai, T.; Iguchi, M.; Yamada, K.-i.; Tomoika, K. Chiral Ligand-Controlled Asymmetric Conjugate Addition of Lithium Amides to Enoates. J. Am. Chem. Soc. 2003, $125,2886$.

(4) Claridge, T. D. W.; Davies, S. G.; Lee, J. A.; Nicholson, R. L.; Roberts, P. M.; Russell, A. J.; Smith, A. D.; Toms, S. M. Highly (E)-Selective Wadsworth-Emmonds Reactions Promoted by Methylmagnesium Bromide. Org. Lett. 2008, 10, 5437.

(5) Kallikat Augustine, J.; Boodappa, C.; Venkatachaliah, S.; Mariappan, A. TiCl4-mediated olefination of aldehydes with acetic acid and alkyl acetates: a stereoselective approach to (E)- $\alpha, \beta$-unsaturated carboxylic acids and esters. Tetrahedron Lett. 2014, 55, 3503.

(6) Yasuda, N.; Hsiao, Y.; Jensen, M. S.; Rivera, N. R.; Yang, C.; Wells, K. M.; Yau, J.; Palucki, M.; Tan, L.; Dormer, P. G.; Volante, R. P.; Hughes, D. L.; Reider, P. J. An Efficient Synthesis of an $\alpha_{\mathrm{v}} \beta_{3}$ Antagonist. J. Org. Chem. 2004, 69, 1959. 
${ }^{1} \mathrm{H}$ NMR (500 MHz, $\mathrm{CDCl}_{3}$ ) of $\mathbf{S I - 1}$

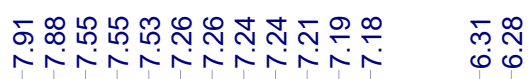
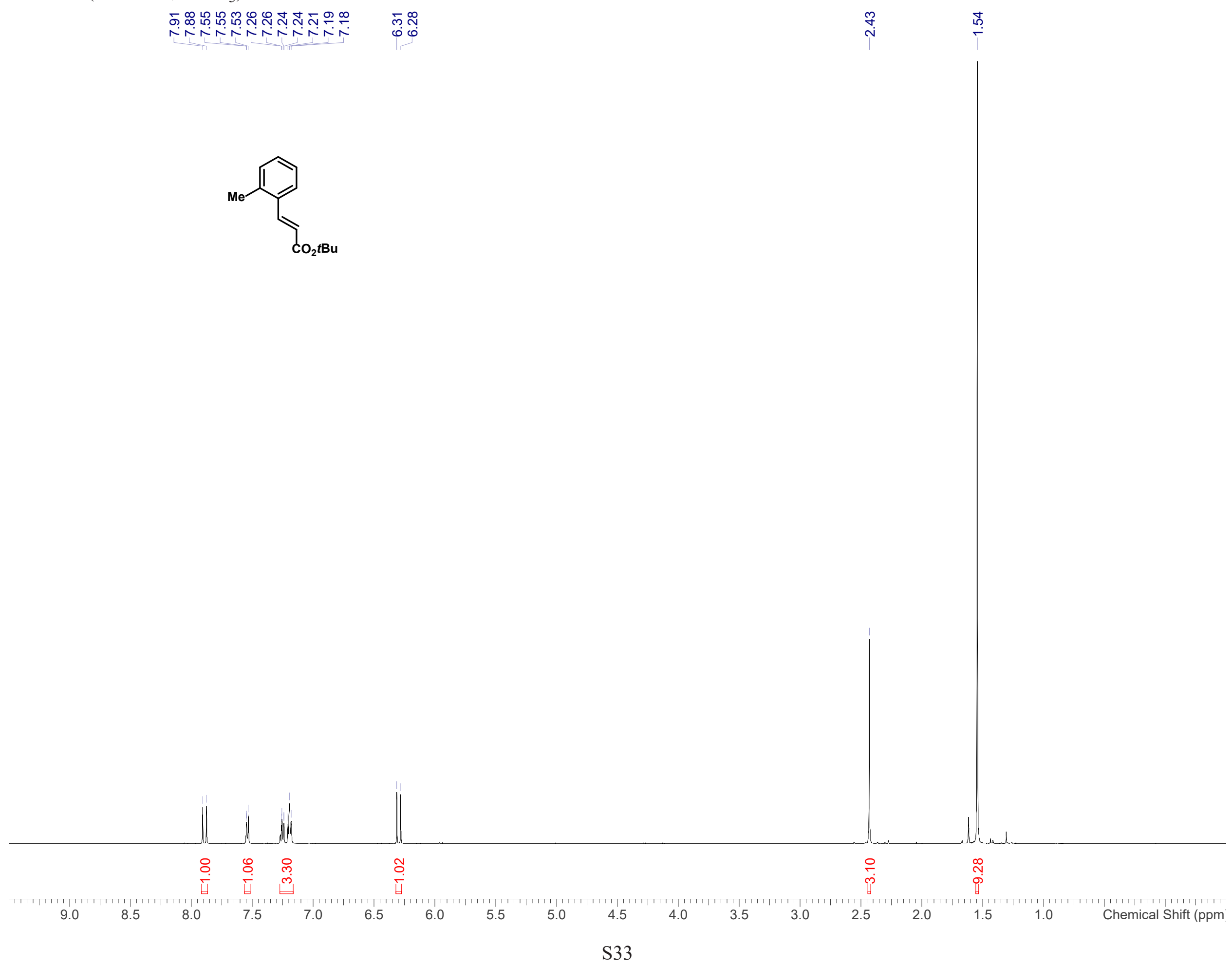
${ }^{13} \mathrm{C}\left\{{ }^{1} \mathrm{H}\right\}$ NMR $\left(126 \mathrm{MHz}, \mathrm{CDCl}_{3}\right)$ of $\mathbf{S I - 1}$

吕

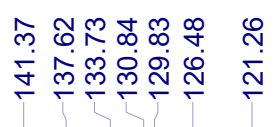

$\underset{\infty}{\infty}$

$\begin{array}{ll}\hat{m} & 8 \\ \stackrel{\infty}{\infty} & \stackrel{\Gamma}{\sim}\end{array}$
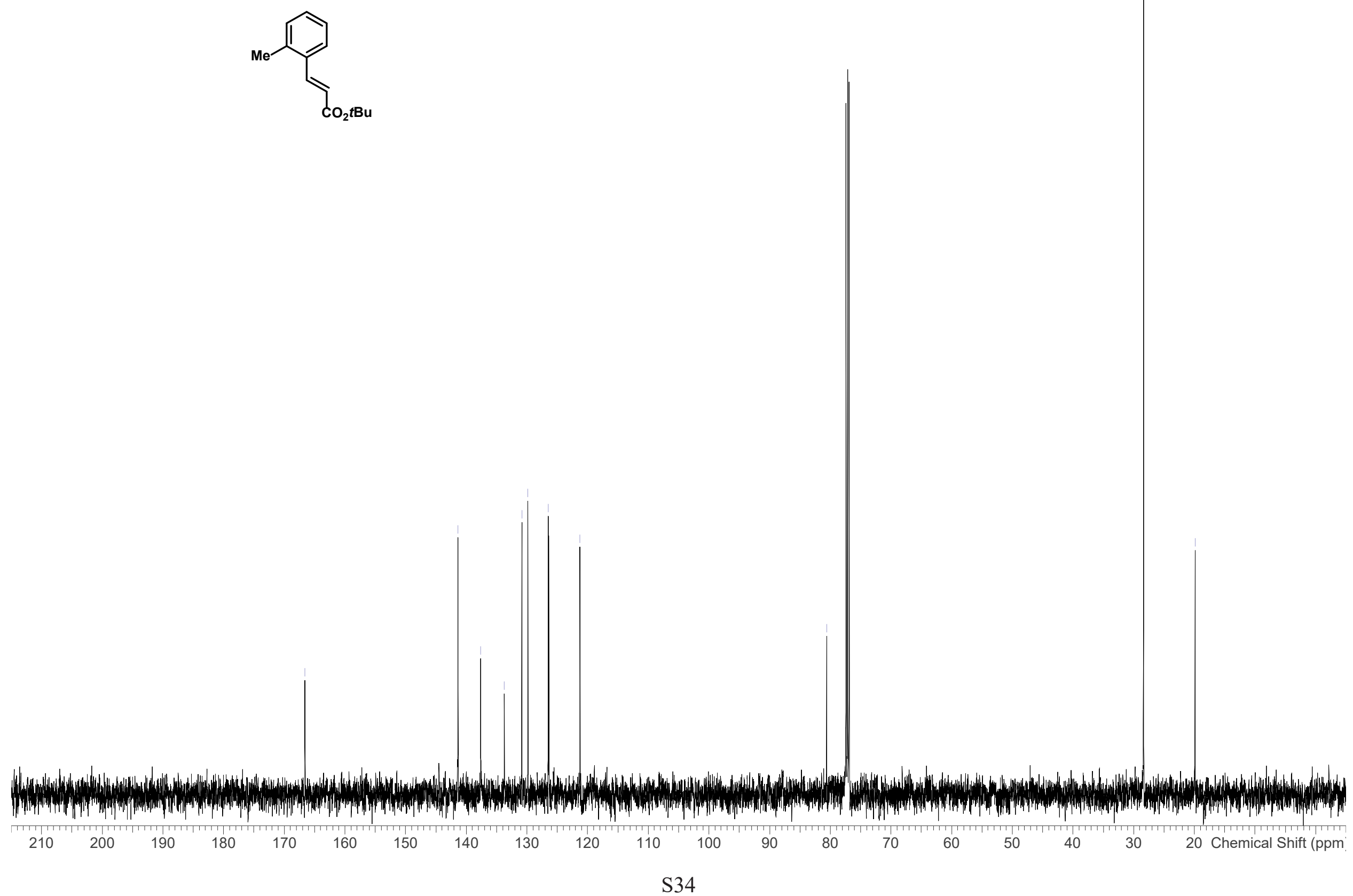

S34 
${ }^{1} \mathrm{H}$ NMR (600 MHz, $\mathrm{CDCl}_{3}$ ) of $\mathbf{S I - 2}$

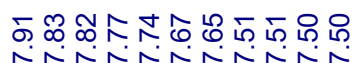
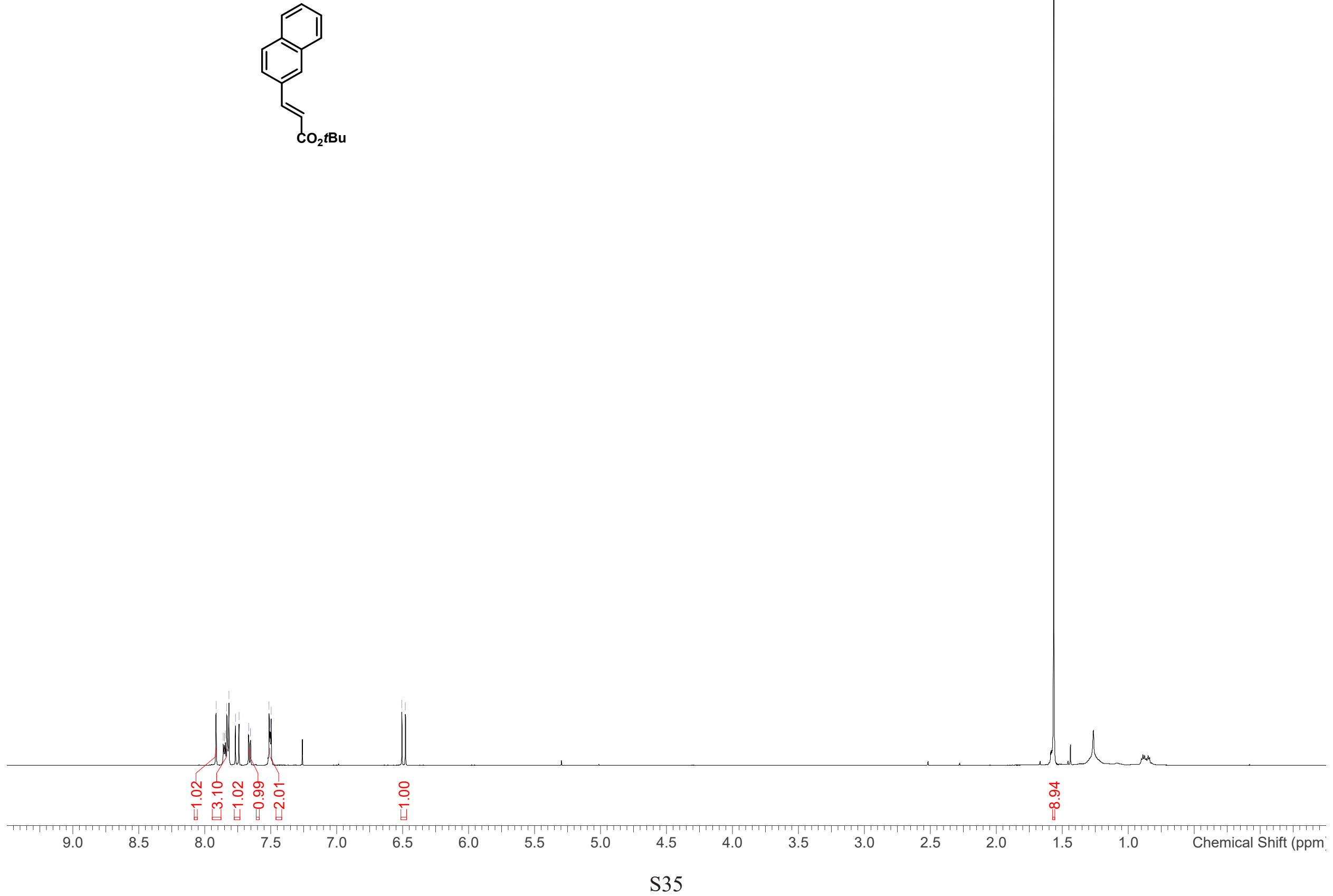
${ }^{13} \mathrm{C}\left\{{ }^{1} \mathrm{H}\right\} \mathrm{NMR}\left(151 \mathrm{MHz}, \mathrm{CDCl}_{3}\right)$ of $\mathbf{S I - 2}$

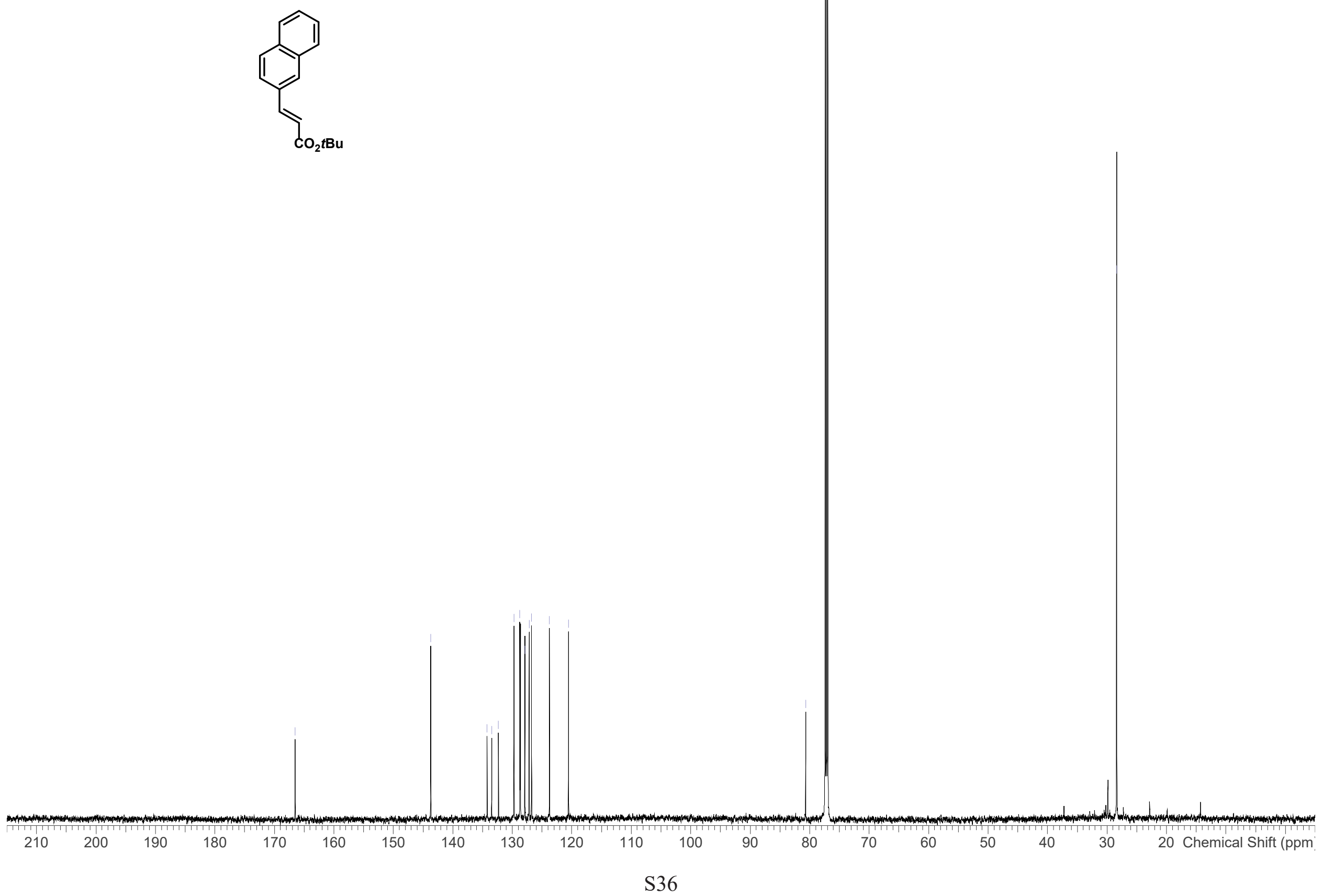


${ }^{1} \mathrm{H}$ NMR $\left(600 \mathrm{MHz}, \mathrm{CDCl}_{3}\right)$ of $\mathbf{S I - 3}$

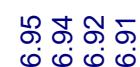

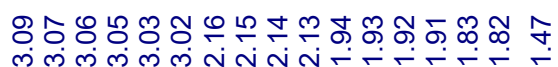
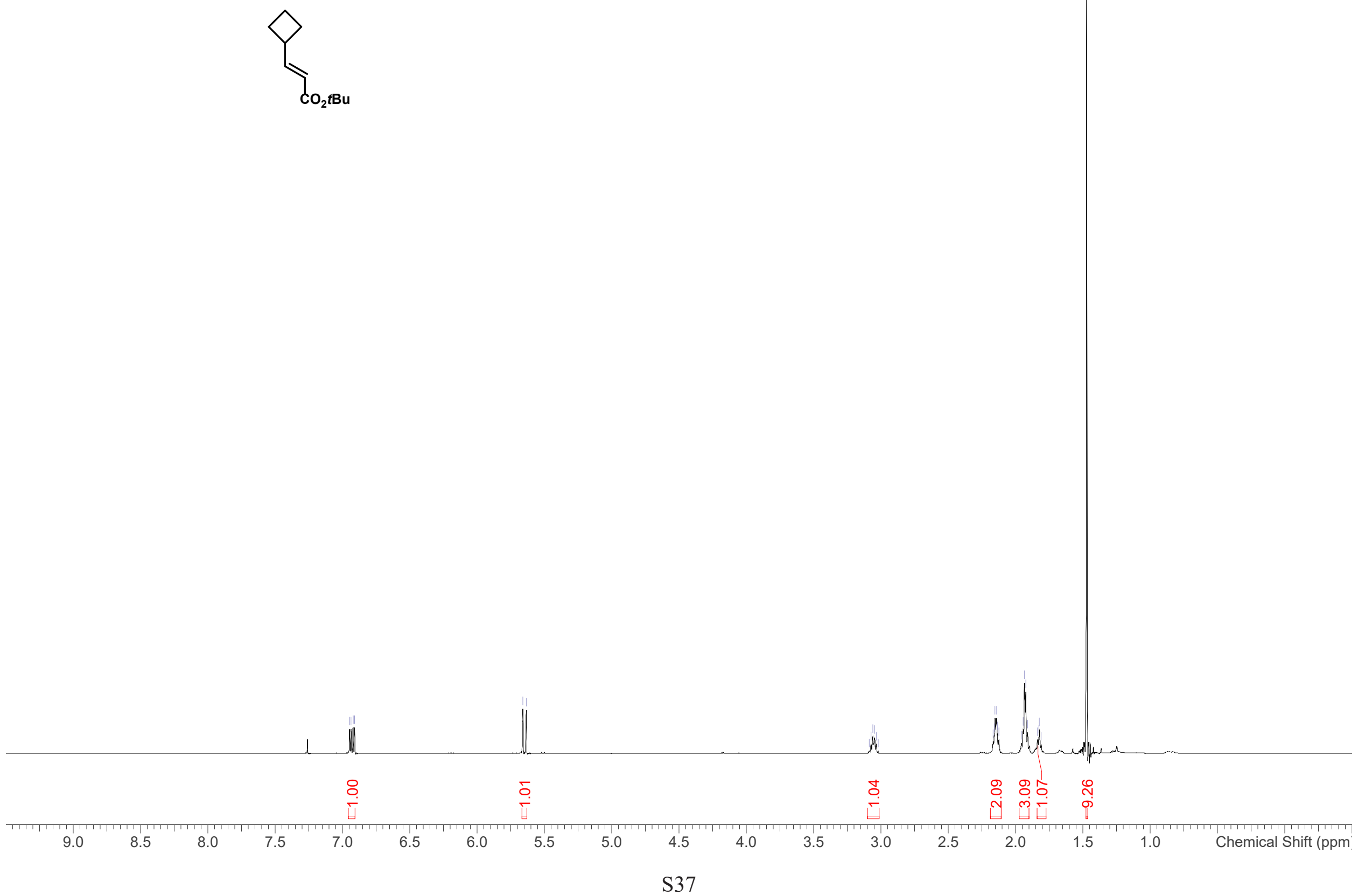
${ }^{13} \mathrm{C}\left\{{ }^{1} \mathrm{H}\right\} \mathrm{NMR}\left(151 \mathrm{MHz}, \mathrm{CDCl}_{3}\right)$ of $\mathbf{S I - 3}$

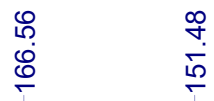

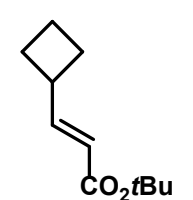


${ }^{1} \mathrm{H}$ NMR $\left(500 \mathrm{MHz}, \mathrm{CDCl}_{3}\right)$ of $\mathbf{S I - 4}$

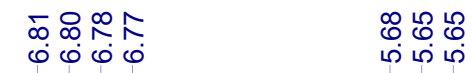

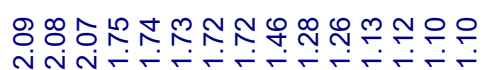

$\underbrace{}_{\mathrm{CO}_{2} \mathrm{tBu}}$

\begin{tabular}{|c|c|c|c|c|c|c|c|c|c|c|c|c|c|c|c|c|c|}
\hline & & & & & & & סَ. & & & & & & & $\stackrel{\text { ஜ̊ }}{\dot{\varphi}}$ & 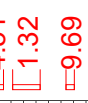 & & \\
\hline 9.0 & 8.5 & 8.0 & 7.5 & 7.0 & 6.5 & 6.0 & 5.5 & 5.0 & 4.5 & 4.0 & 3.5 & 3.0 & 2.5 & 2.0 & 1.5 & 1.0 & Chemical Shift (ppm \\
\hline
\end{tabular}


${ }^{13} \mathrm{C}\left\{{ }^{1} \mathrm{H}\right\} \mathrm{NMR}\left(126 \mathrm{MHz}, \mathrm{CDCl}_{3}\right)$ of SI-4

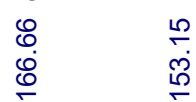

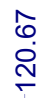

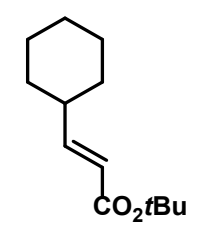


${ }^{1} \mathrm{H}$ NMR $\left(600 \mathrm{MHz}, \mathrm{CDCl}_{3}\right)$ of SI-5

\begin{tabular}{|c|c|c|c|c|}
\hline 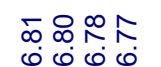 & 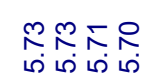 & 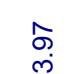 & 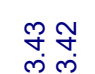 & ⿹ \\
\hline
\end{tabular}
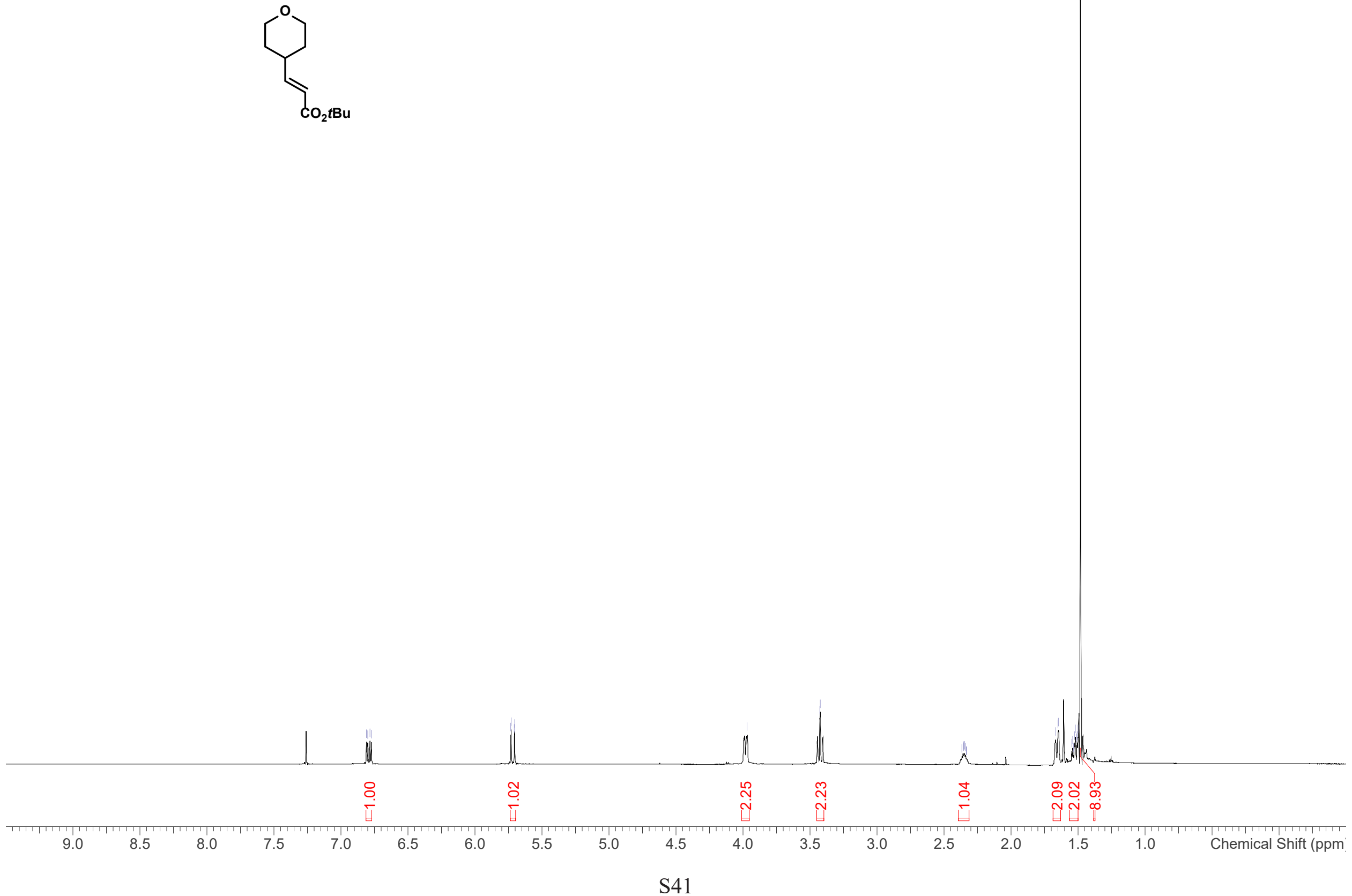
${ }^{13} \mathrm{C}\left\{{ }^{1} \mathrm{H}\right\}$ NMR $\left(151 \mathrm{MHz}, \mathrm{CDCl}_{3}\right)$ of $\mathbf{S I - 5}$

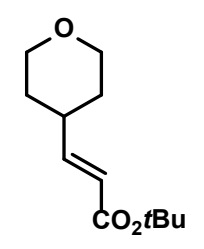

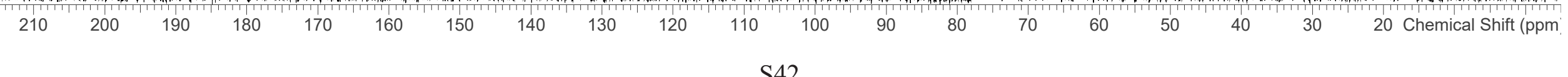


${ }^{1} \mathrm{H}$ NMR $\left(500 \mathrm{MHz}, \mathrm{CDCl}_{3}\right)$ of SI-6

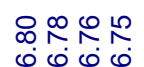

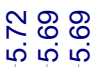

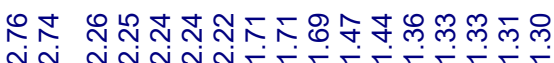

$\xi$

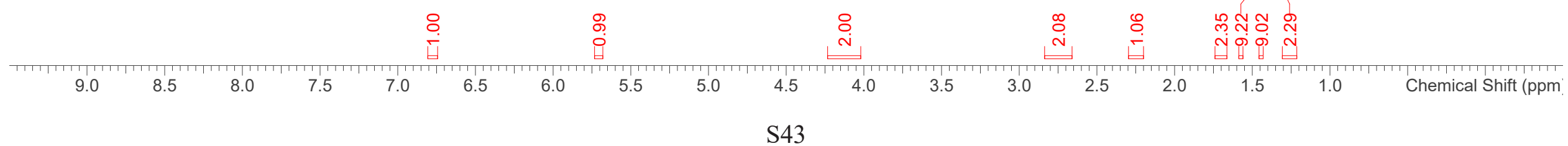


${ }^{13} \mathrm{C}\left\{{ }^{1} \mathrm{H}\right\} \mathrm{NMR}\left(126 \mathrm{MHz}, \mathrm{CDCl}_{3}\right)$ of SI-6

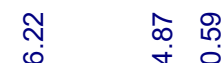

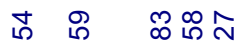

$\because \quad \stackrel{6}{\square}$

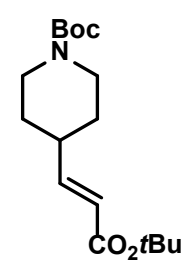

44 
${ }^{1} \mathrm{H}$ NMR (500 MHz, $\mathrm{CDCl}_{3}$ ) of $\mathbf{S I - 7}$

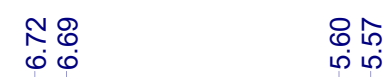

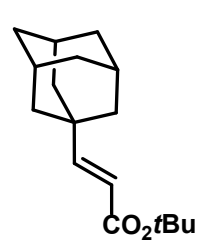

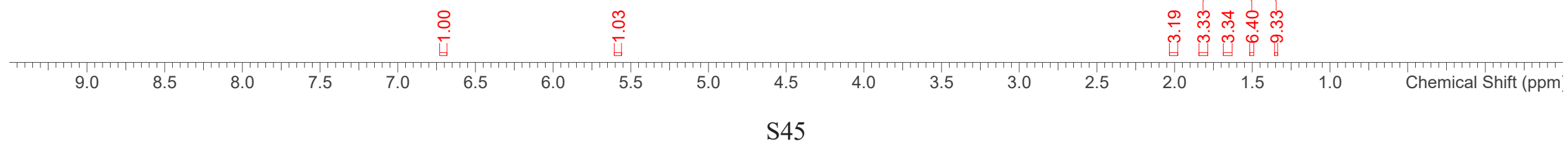


${ }^{13} \mathrm{C}\left\{{ }^{1} \mathrm{H}\right\} \mathrm{NMR}\left(126 \mathrm{MHz}, \mathrm{CDCl}_{3}\right)$ of $\mathbf{S I - 7}$

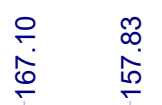

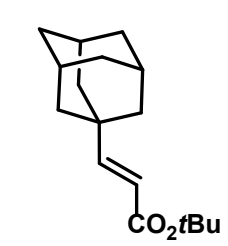

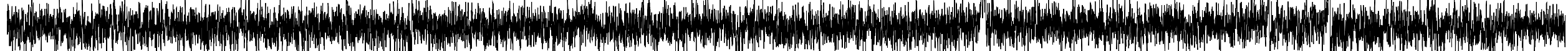

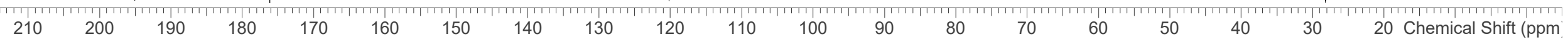


${ }^{1} \mathrm{H}$ NMR $\left(500 \mathrm{MHz}, \mathrm{CDCl}_{3}\right)$ of $\mathbf{S I - 8}$

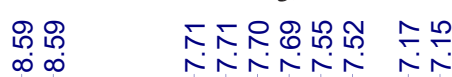
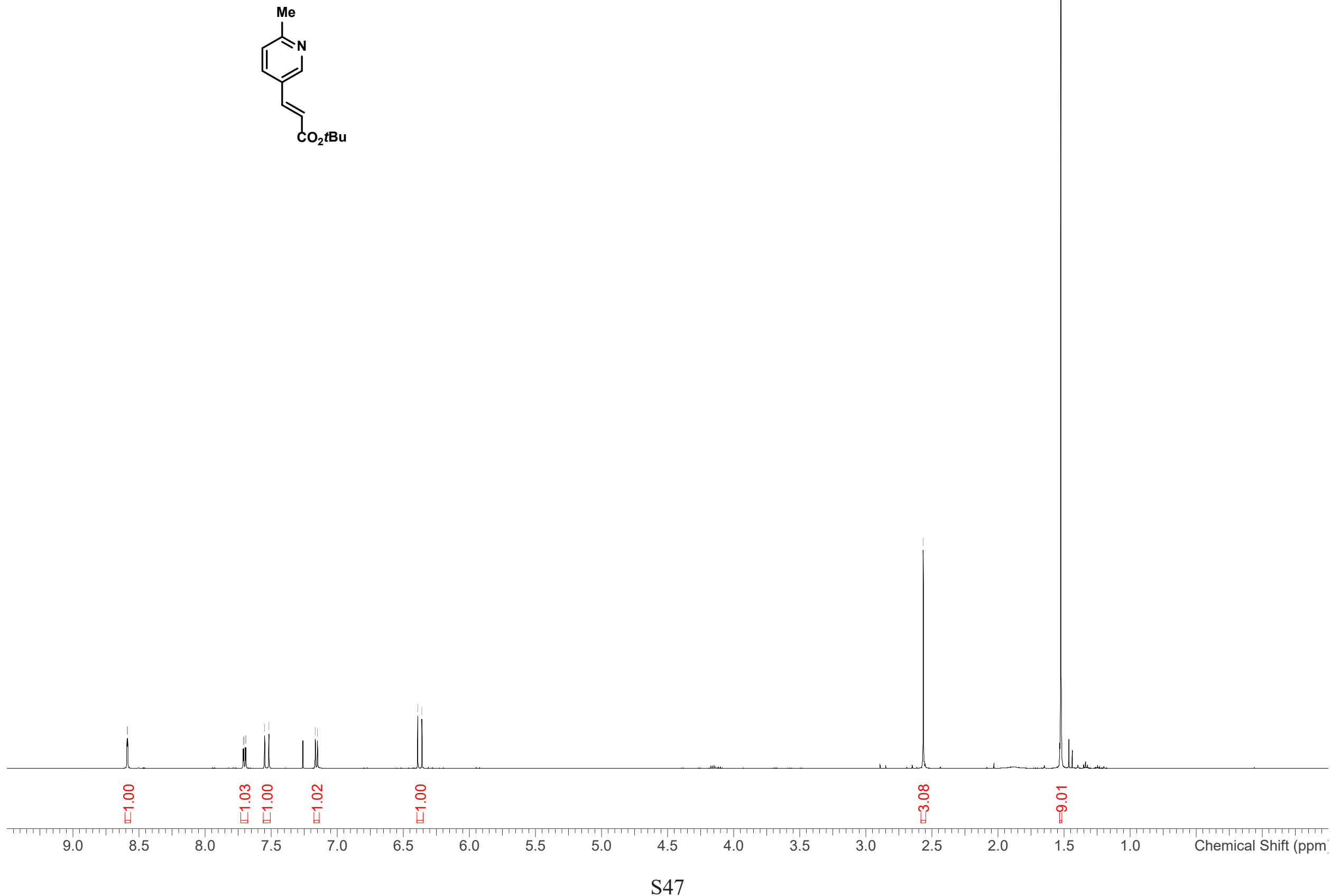
${ }^{13} \mathrm{C}\left\{{ }^{1} \mathrm{H}\right\}$ NMR $\left(126 \mathrm{MHz}, \mathrm{CDCl}_{3}\right)$ of $\mathbf{S I - 8}$

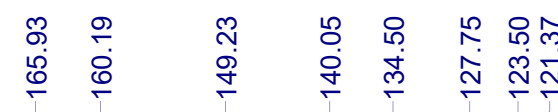

m. โุ

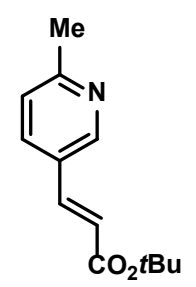

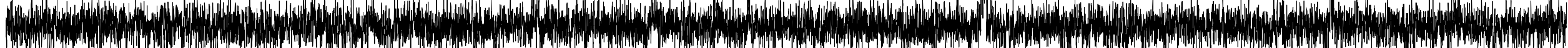


${ }^{1} \mathrm{H}$ NMR $\left(600 \mathrm{MHz}, \mathrm{CDCl}_{3}\right)$ of SI-9

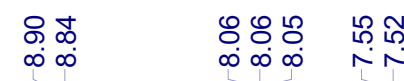
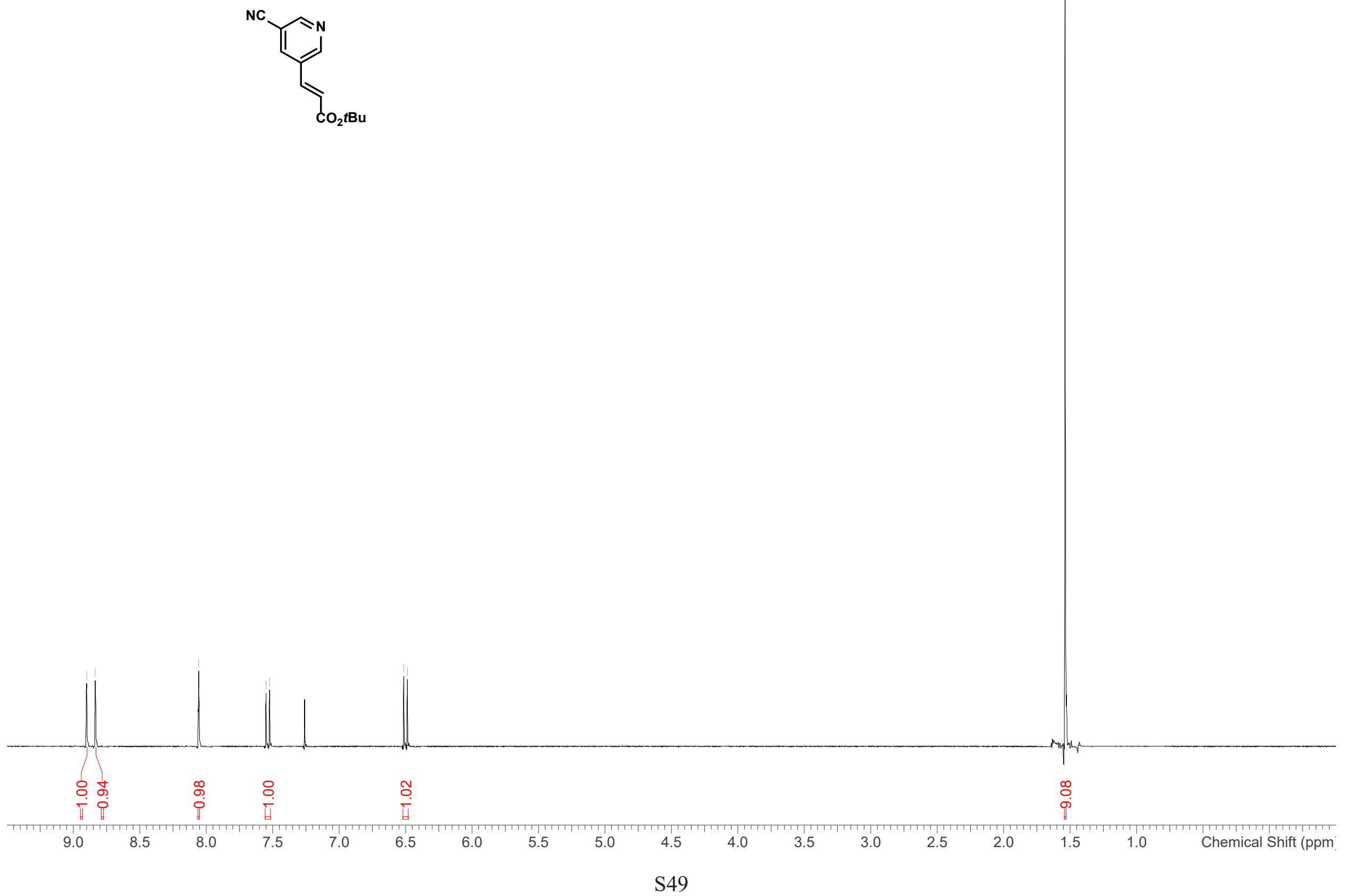
${ }^{13} \mathrm{C}\left\{{ }^{1} \mathrm{H}\right\} \mathrm{NMR}\left(151 \mathrm{MHz}, \mathrm{CDCl}_{3}\right)$ of SI-9

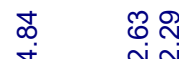

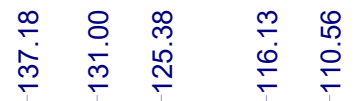

$\frac{\infty}{\infty}$

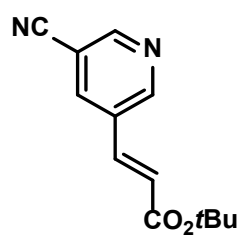


${ }^{1} \mathrm{H}-{ }^{13} \mathrm{C}$ HSQC NMR $\left(\mathrm{CDCl}_{3}\right)$ of $\mathbf{S I - 9}$

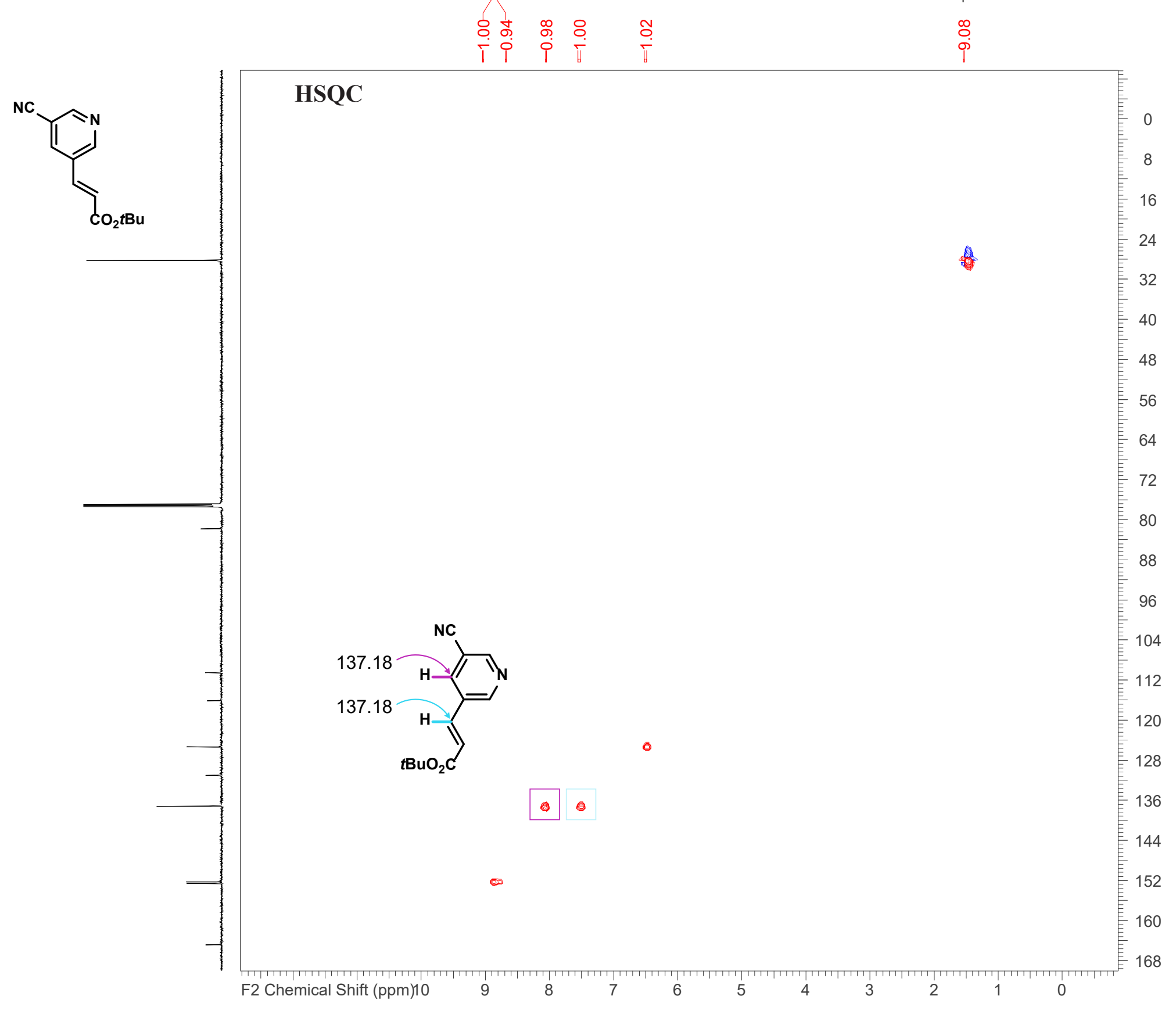


${ }^{1} \mathrm{H}$ NMR $\left(600 \mathrm{MHz}, \mathrm{CDCl}_{3}\right)$ of SI-10

$\stackrel{\substack{R \\ \infty}}{\infty}$

守

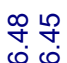
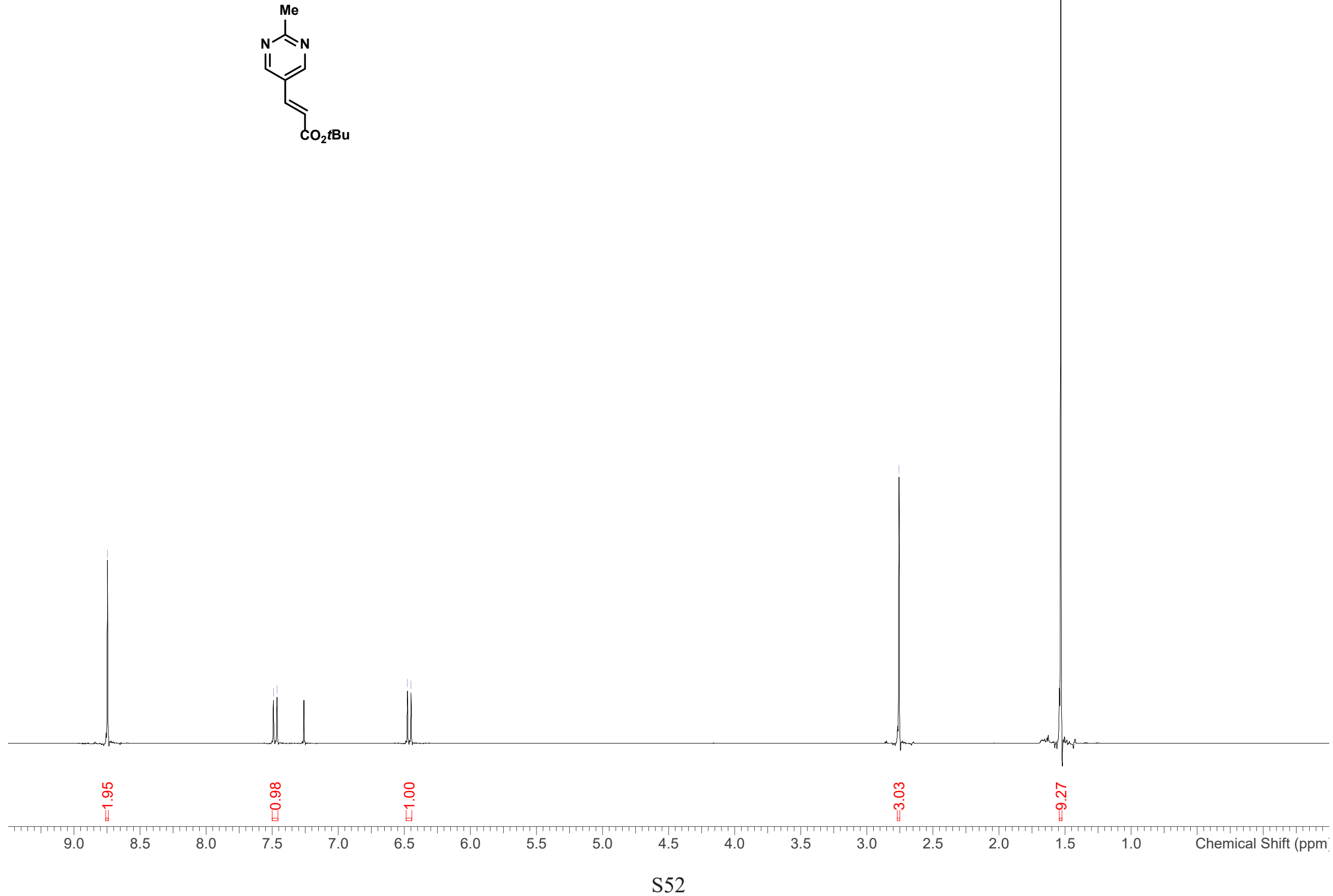
${ }^{13} \mathrm{C}\left\{{ }^{1} \mathrm{H}\right\}$ NMR $\left(151 \mathrm{MHz}, \mathrm{CDCl}_{3}\right)$ of SI-10

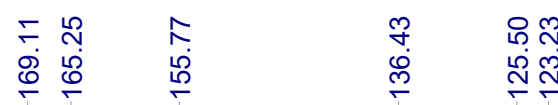

Me<smiles>CCOC(=O)C=Cc1cnc(C)nc1</smiles> 
${ }^{1} \mathrm{H}$ NMR $\left(600 \mathrm{MHz}, \mathrm{CDCl}_{3}\right)$ of SI-11

芯

守尔

$\begin{array}{ll}\infty & 0 \\ m & 0 \\ 0 & 0 \\ 0 & 0\end{array}$
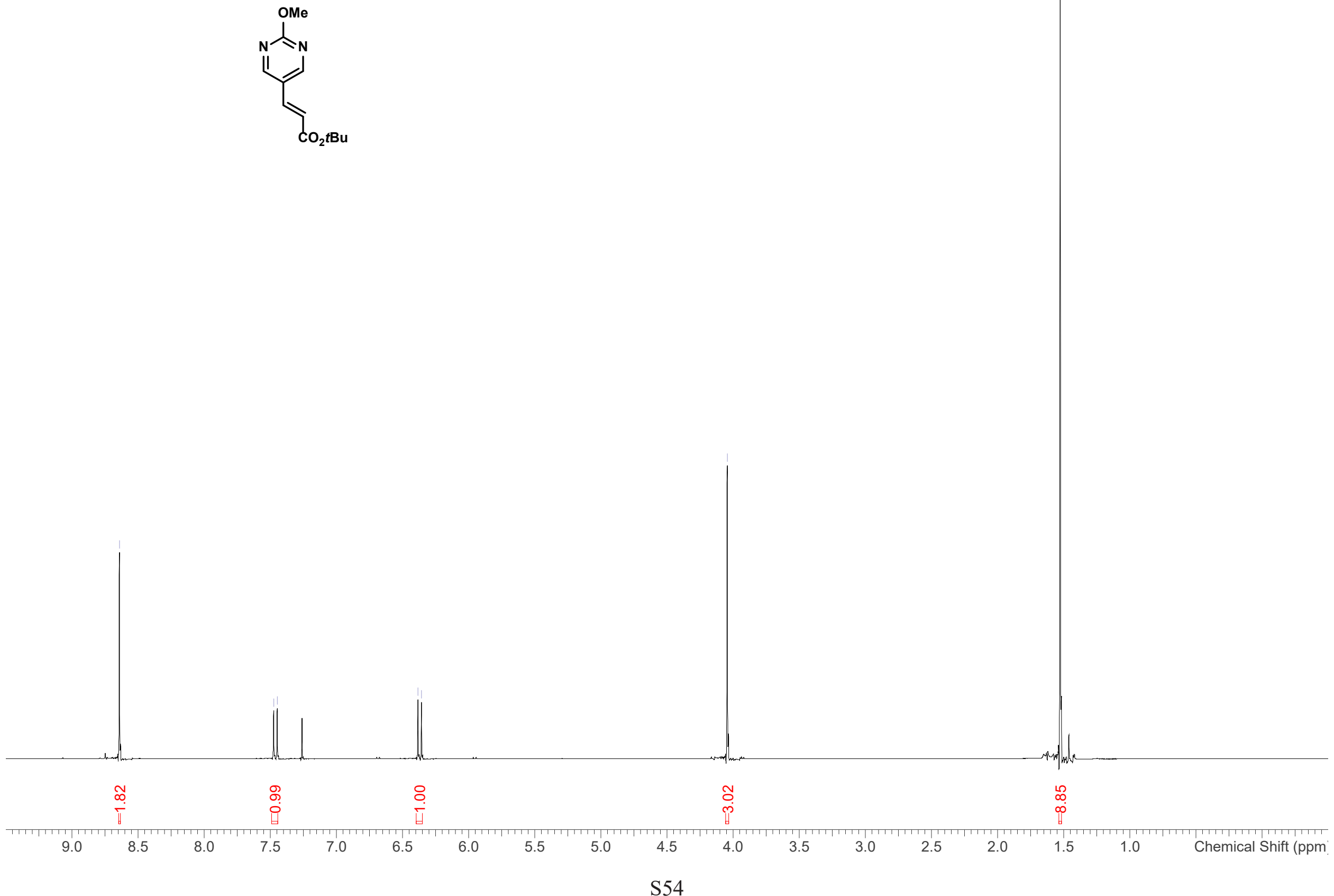
${ }^{13} \mathrm{C}\left\{{ }^{1} \mathrm{H}\right\}$ NMR $\left(151 \mathrm{MHz}, \mathrm{CDCl}_{3}\right)$ of SI-11

$\forall\llcorner$

잉

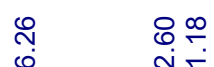

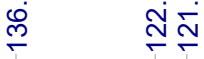

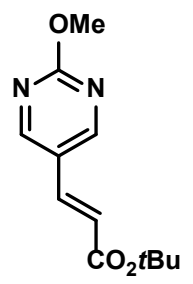


${ }^{1} \mathrm{H}$ NMR $\left(500 \mathrm{MHz}, \mathrm{CDCl}_{3}\right)$ of SI-12

क⿻

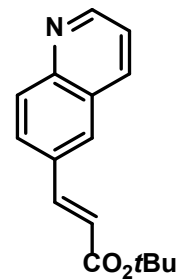

\begin{tabular}{|c|c|c|c|c|c|c|c|c|c|c|c|c|c|c|c|c|c|}
\hline $\begin{array}{l}8 \\
\stackrel{8}{0} \\
0\end{array}$ & & 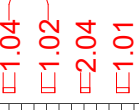 & $\begin{array}{l}\text { बे } \\
\text { 부 }\end{array}$ & & $\frac{8}{\dot{\zeta}}$ & & & & & & & & & & $\begin{array}{l}\forall \\
\infty \\
\infty \\
0 \\
\|\end{array}$ & & \\
\hline 9.0 & 8.5 & 8.0 & 7.5 & 7.0 & 6.5 & 6.0 & 5.5 & 5.0 & 4.5 & 4.0 & 3.5 & 3.0 & 2.5 & 2.0 & 1.5 & 1.0 & Chemical Shift (ppm \\
\hline
\end{tabular}


${ }^{13} \mathrm{C}\left\{{ }^{1} \mathrm{H}\right\}$ NMR $\left(126 \mathrm{MHz}, \mathrm{CDCl}_{3}\right)$ of $\mathbf{S I - 1 2}$

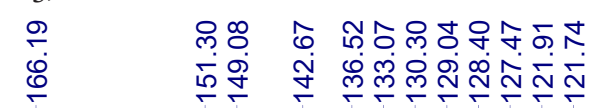

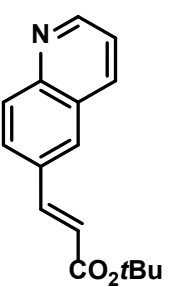

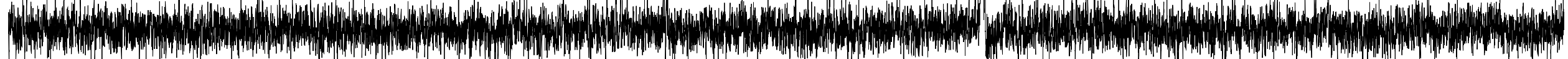


${ }^{1} \mathrm{H}$ NMR $\left(500 \mathrm{MHz}, \mathrm{CDCl}_{3}\right)$ of 6

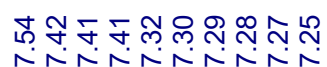

ขูก รลำ

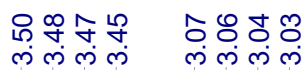

$\stackrel{m}{\stackrel{m}{-}}$
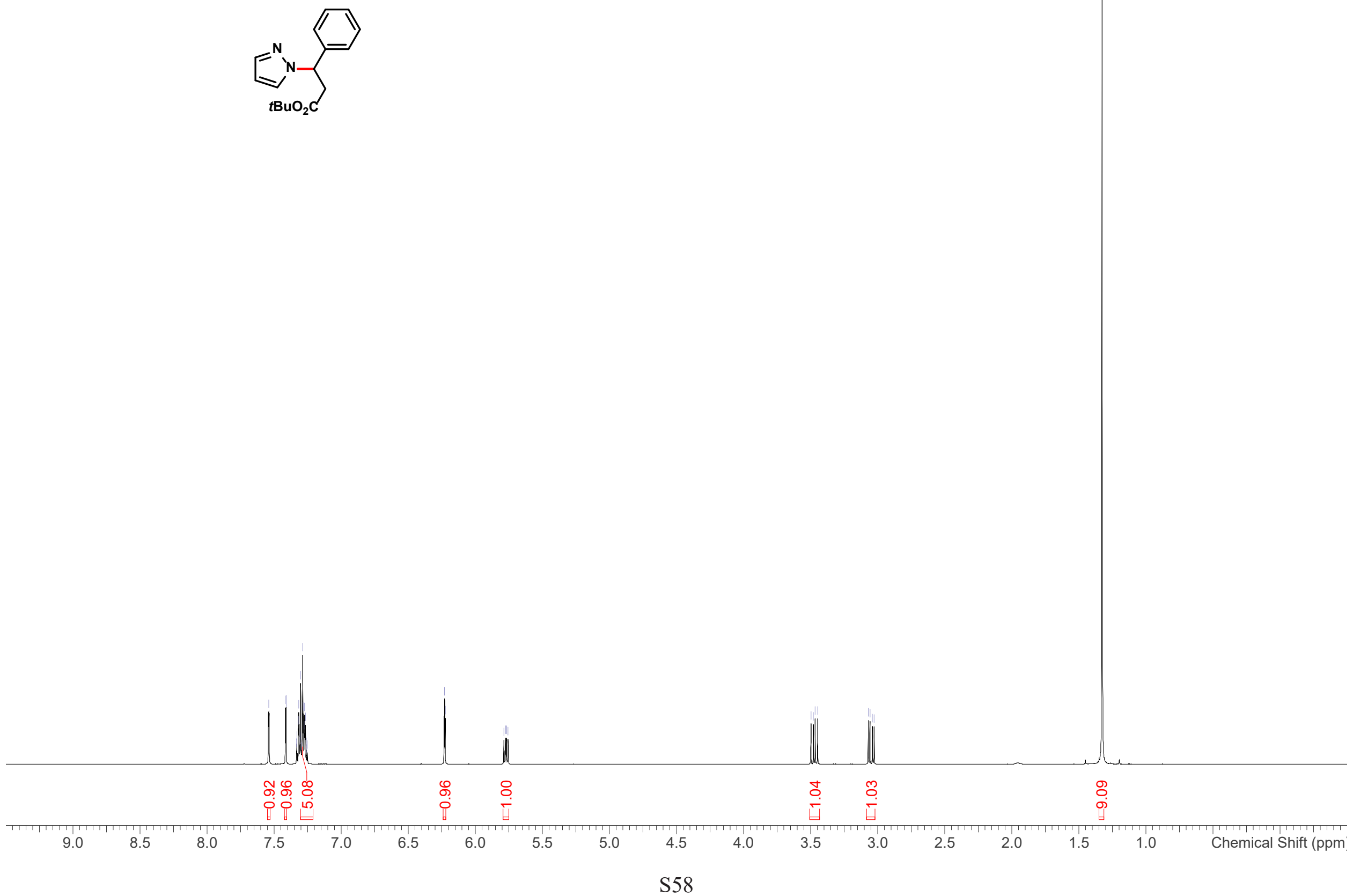
$\left.{ }^{13} \mathrm{C}^{1} \mathrm{H}\right\} \mathrm{NMR}\left(126 \mathrm{MHz}, \mathrm{CDCl}_{3}\right)$ of 6

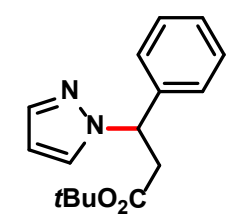


${ }^{1} \mathrm{H} \mathrm{NMR}\left(500 \mathrm{MHz}, \mathrm{CDCl}_{3}\right)$ of $\mathbf{7 a}$

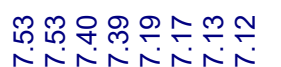

ละกล

000 ம்

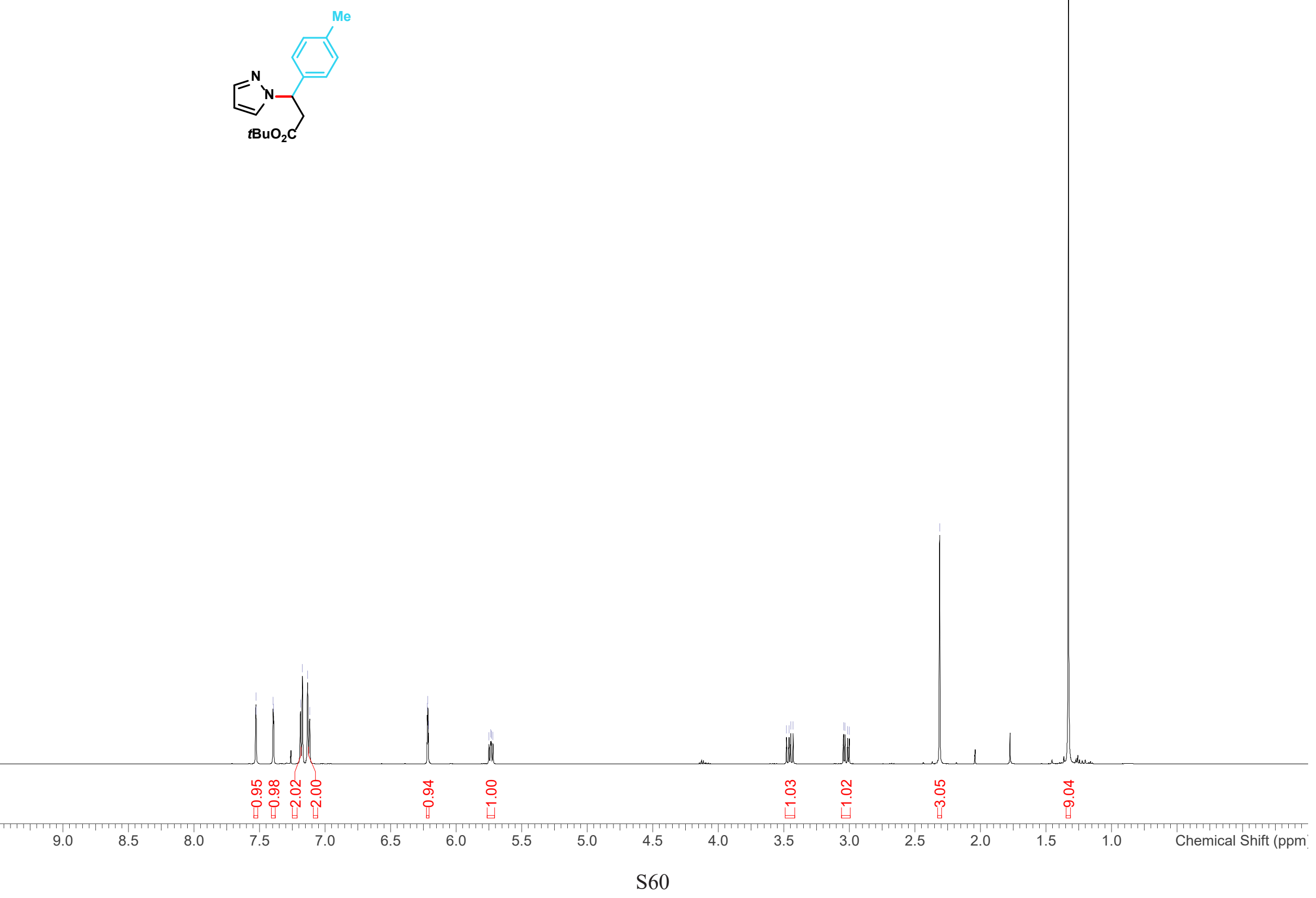

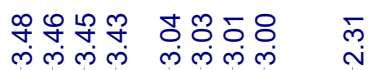

$\stackrel{m}{\sim}$ 
${ }^{13} \mathrm{C}\left\{{ }^{1} \mathrm{H}\right\} \mathrm{NMR}\left(126 \mathrm{MHz}, \mathrm{CDCl}_{3}\right)$ of $7 \mathbf{a}$

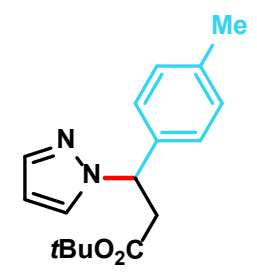

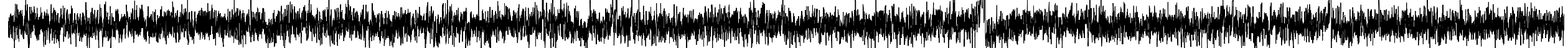


${ }^{1} \mathrm{H}$ NMR $\left(600 \mathrm{MHz}, \mathrm{CDCl}_{3}\right)$ of $\mathbf{7 b}$

수소ำ
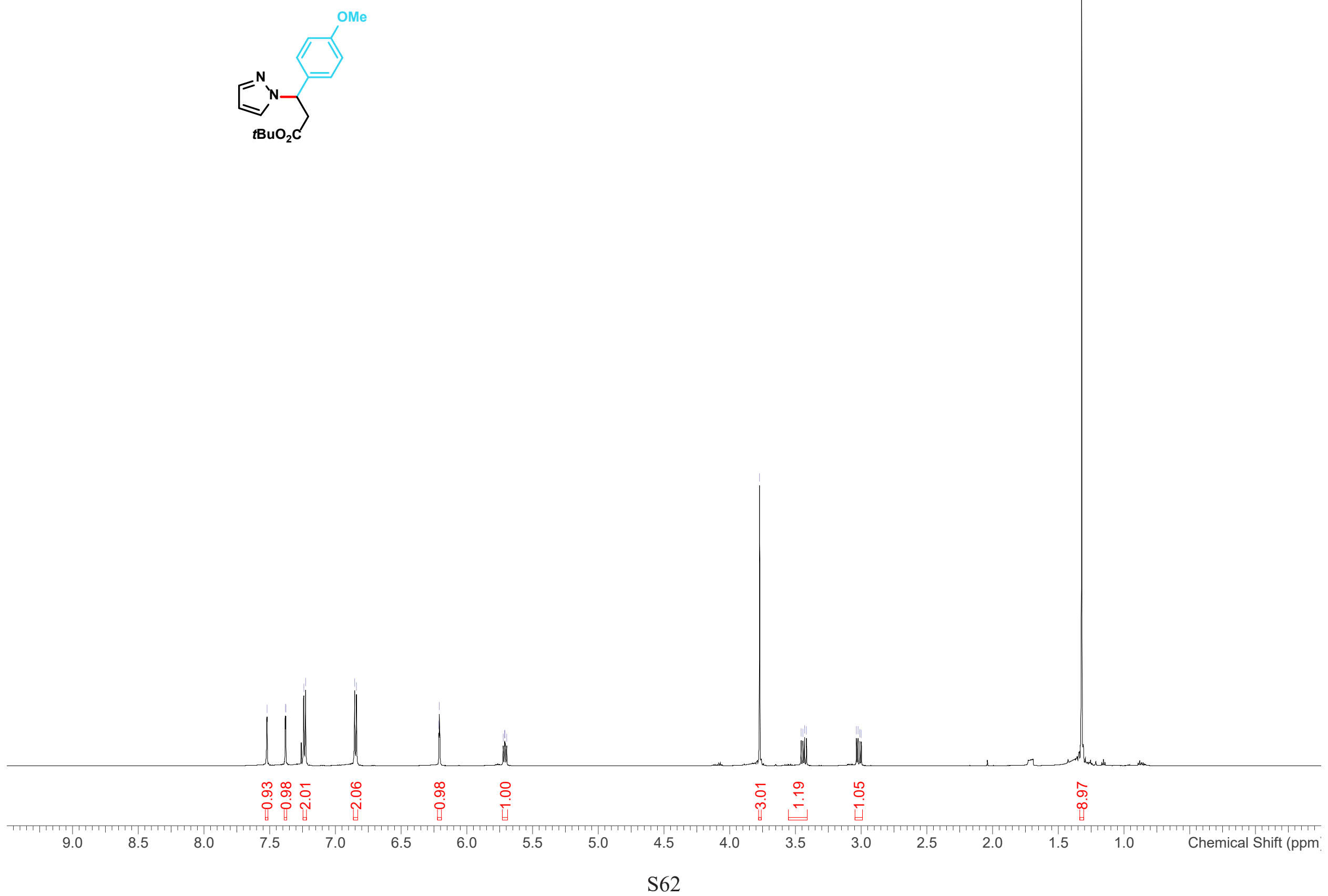
${ }^{13} \mathrm{C}\left\{{ }^{1} \mathrm{H}\right\} \mathrm{NMR}\left(151 \mathrm{MHz}, \mathrm{CDCl}_{3}\right)$ of $\mathbf{7 b}$

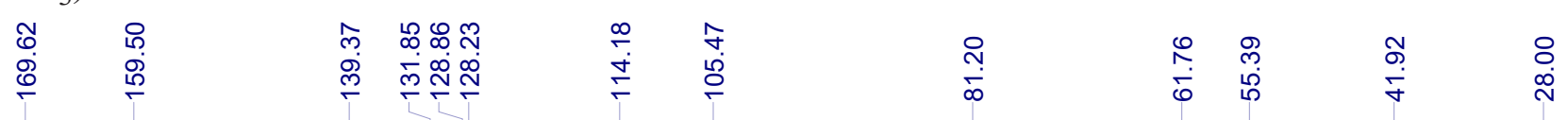

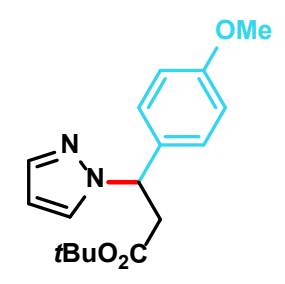


${ }^{1} \mathrm{H} \mathrm{NMR}\left(600 \mathrm{MHz}, \mathrm{CDCl}_{3}\right)$ of $7 \mathrm{c}$

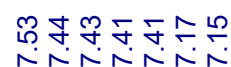

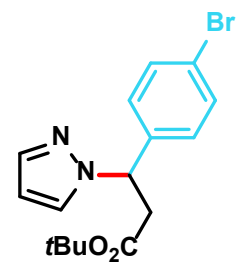

\begin{tabular}{|c|c|c|c|c|c|c|c|c|c|c|c|c|c|c|c|c|c|}
\hline & & & 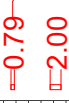 & & & & $\begin{array}{l}\stackrel{8}{0} \\
\text { ப் }\end{array}$ & & & & $\stackrel{\text { ஜூ }}{\dot{\varphi}}$ & $\begin{array}{l}\ddot{\varrho} \\
\dot{\square}\end{array}$ & & & & & \\
\hline 9.0 & 8.5 & 8.0 & 7.5 & 7.0 & 6.5 & 6.0 & 5.5 & 5.0 & 4.5 & 4.0 & 3.5 & 3.0 & 2.5 & 2.0 & 1.5 & 1.0 & Chemical Shift (ppm \\
\hline
\end{tabular}


${ }^{13} \mathrm{C}\left\{{ }^{1} \mathrm{H}\right\} \mathrm{NMR}\left(151 \mathrm{MHz}, \mathrm{CDCl}_{3}\right)$ of $7 \mathbf{c}$

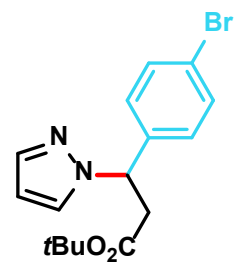


${ }^{1} \mathrm{H} \mathrm{NMR}\left(500 \mathrm{MHz}, \mathrm{CDCl}_{3}\right)$ of $\mathbf{7 d}$

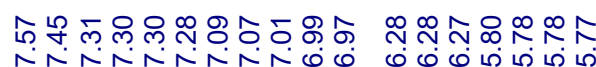

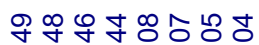
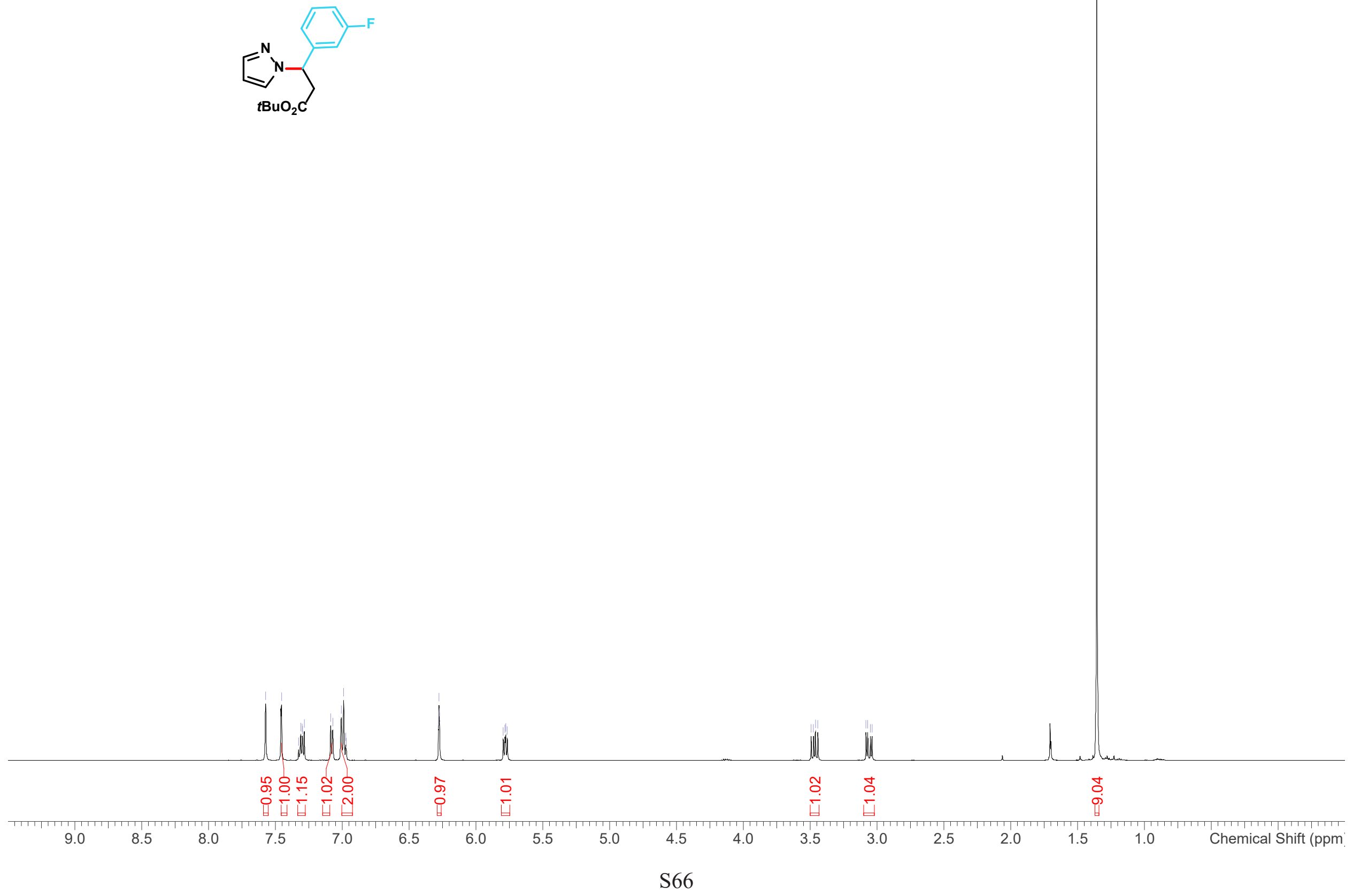
${ }^{13} \mathrm{C}\left\{{ }^{1} \mathrm{H}\right\} \mathrm{NMR}\left(126 \mathrm{MHz}, \mathrm{CDCl}_{3}\right)$ of 7d

$\stackrel{\infty}{\circ}$ \&

ஜㅇํㅇ

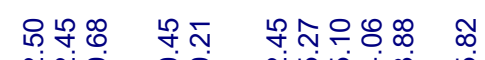

궘ำ

$\stackrel{\infty}{\infty}$

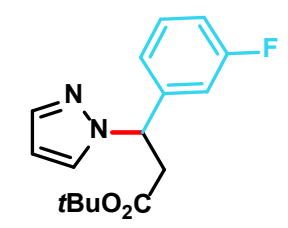

How

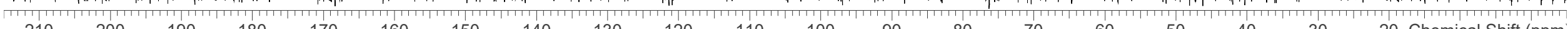
$210 \quad 200$ 190 80 160 150 140 130 120 110 
${ }^{19} \mathrm{~F}$ NMR $\left(471 \mathrm{MHz}, \mathrm{CDCl}_{3}\right)$ of $\mathbf{7 d}$

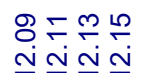

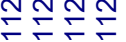

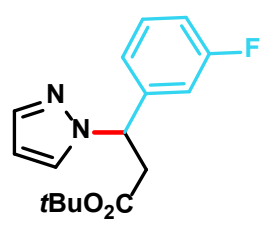


${ }^{1} \mathrm{H} \mathrm{NMR}\left(500 \mathrm{MHz}, \mathrm{CDCl}_{3}\right)$ of $7 \mathbf{e}$

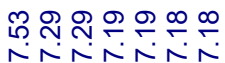

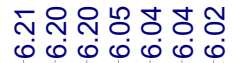

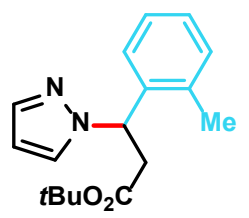

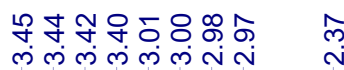

$\stackrel{\text { m }}{\stackrel{2}{.}}$

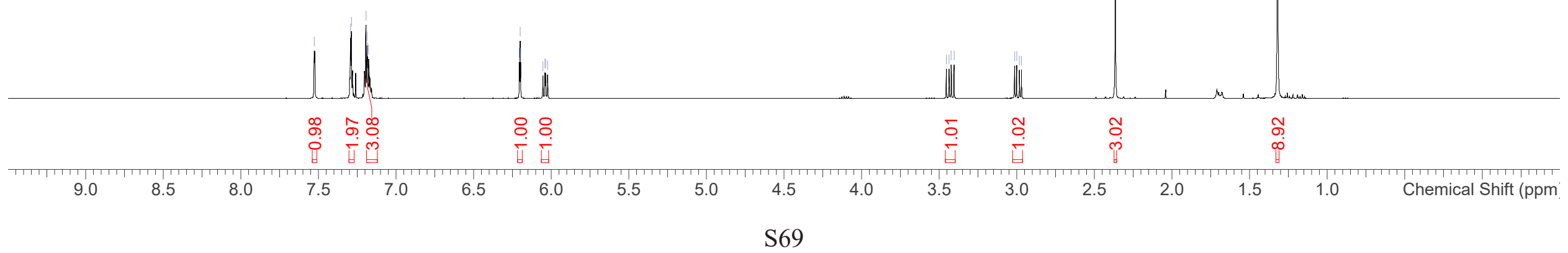


${ }^{13} \mathrm{C}\left\{{ }^{1} \mathrm{H}\right\} \mathrm{NMR}\left(126 \mathrm{MHz}, \mathrm{CDCl}_{3}\right)$ of $7 \mathbf{e}$

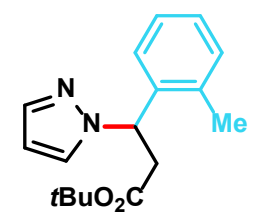


${ }^{1} \mathrm{H}$ NMR $\left(500 \mathrm{MHz}, \mathrm{CDCl}_{3}\right)$ of $\mathbf{7 f}$

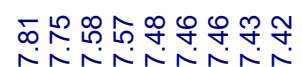

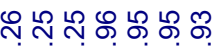

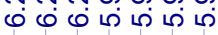

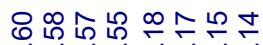

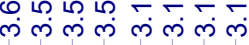

$\stackrel{\text { m }}{\longrightarrow}$
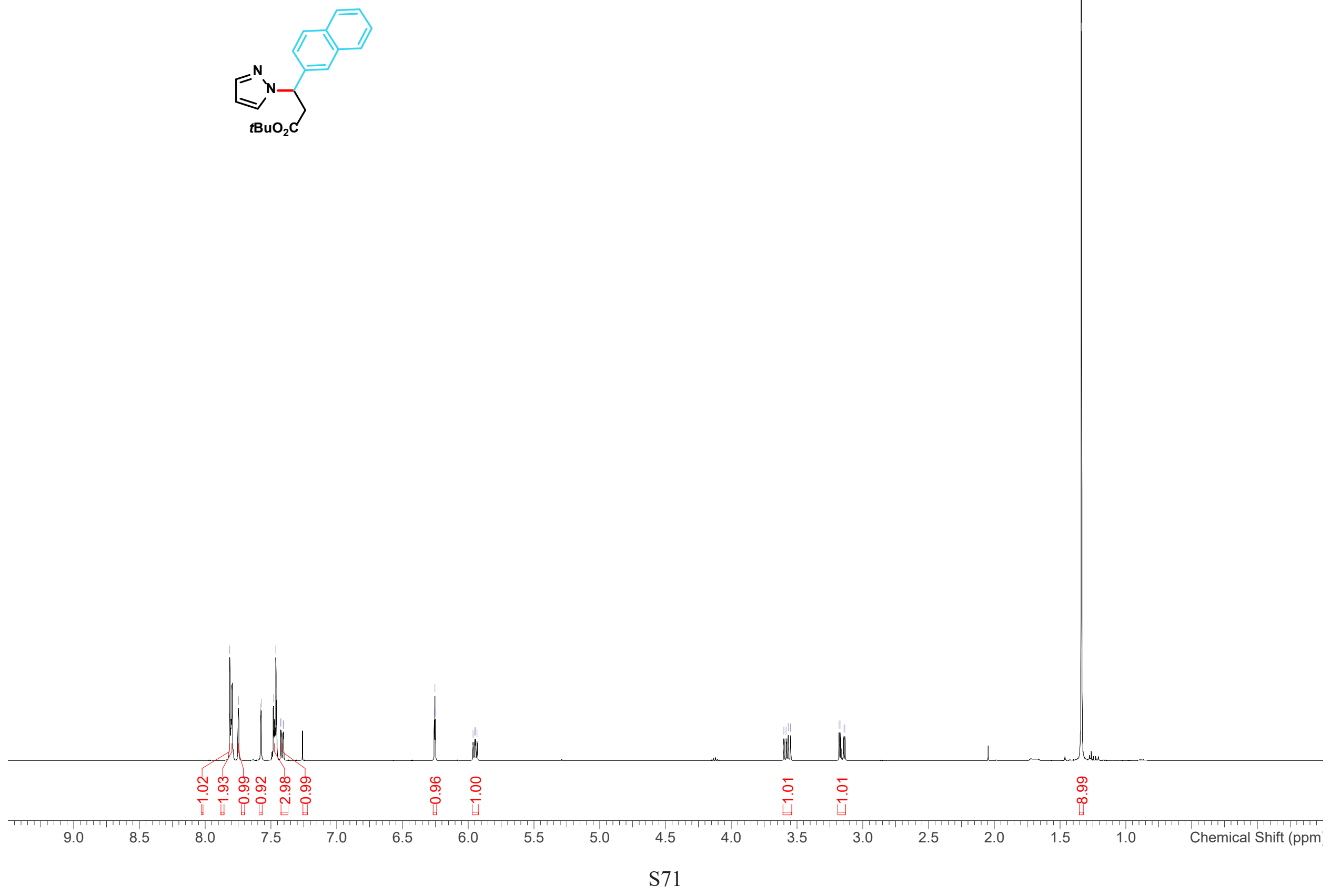
${ }^{13} \mathrm{C}\left\{{ }^{1} \mathrm{H}\right\} \mathrm{NMR}\left(126 \mathrm{MHz}, \mathrm{CDCl}_{3}\right)$ of $7 \mathbf{f}$

舟 ำ

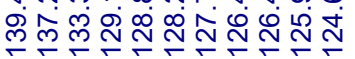

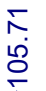

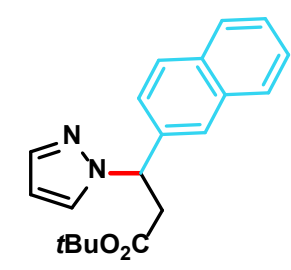

Whathow 
${ }^{1} \mathrm{H} \mathrm{NMR}\left(600 \mathrm{MHz}, \mathrm{CDCl}_{3}\right)$ of $\mathbf{7 g}$
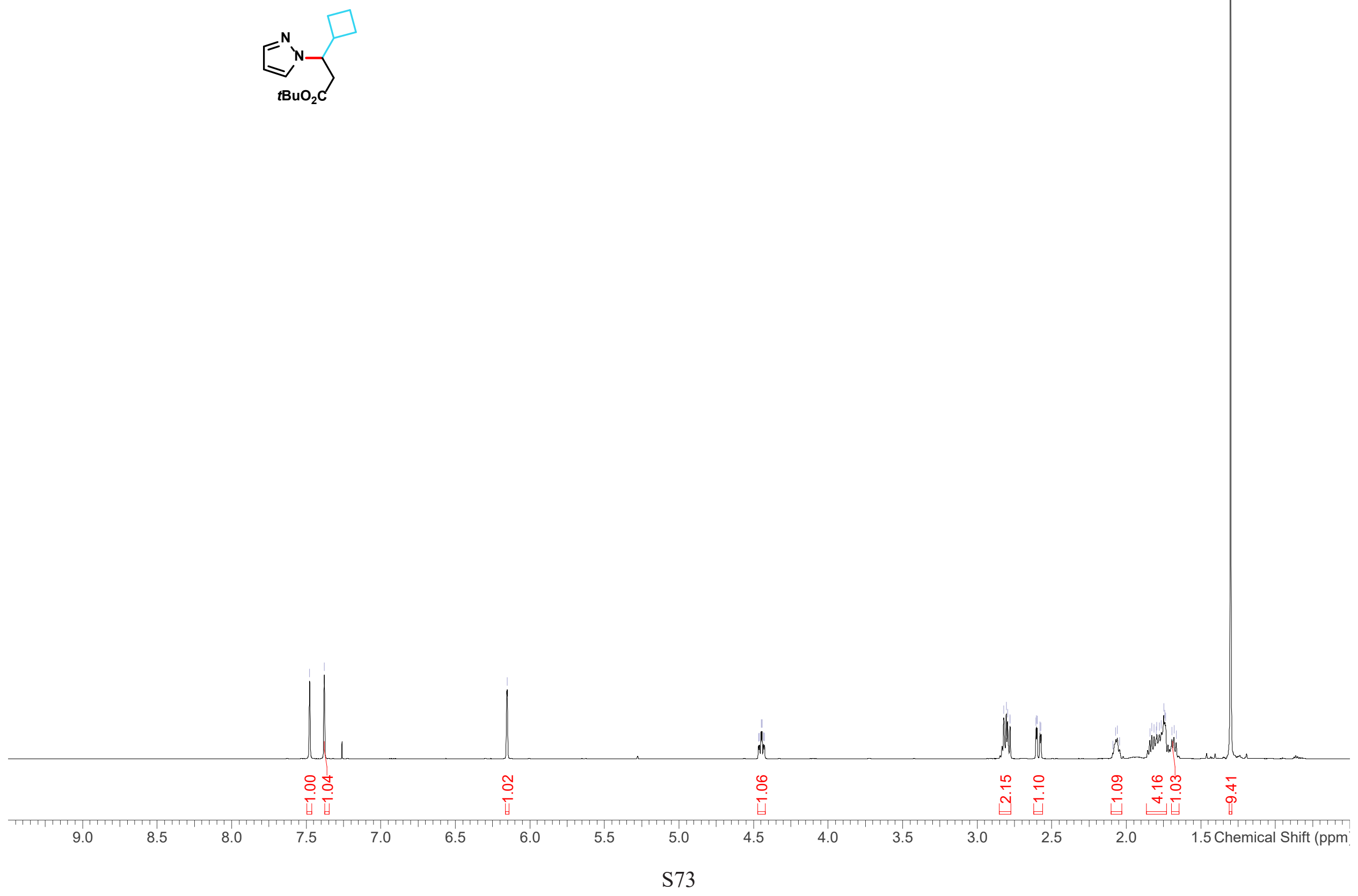
${ }^{13} \mathrm{C}\left\{{ }^{1} \mathrm{H}\right\} \mathrm{NMR}\left(151 \mathrm{MHz}, \mathrm{CDCl}_{3}\right)$ of $\mathbf{7 g}$

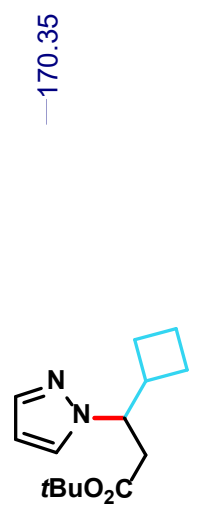

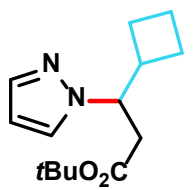


${ }^{1} \mathrm{H}$ NMR $\left(500 \mathrm{MHz}, \mathrm{CDCl}_{3}\right)$ of $\mathbf{7 h}$

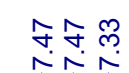
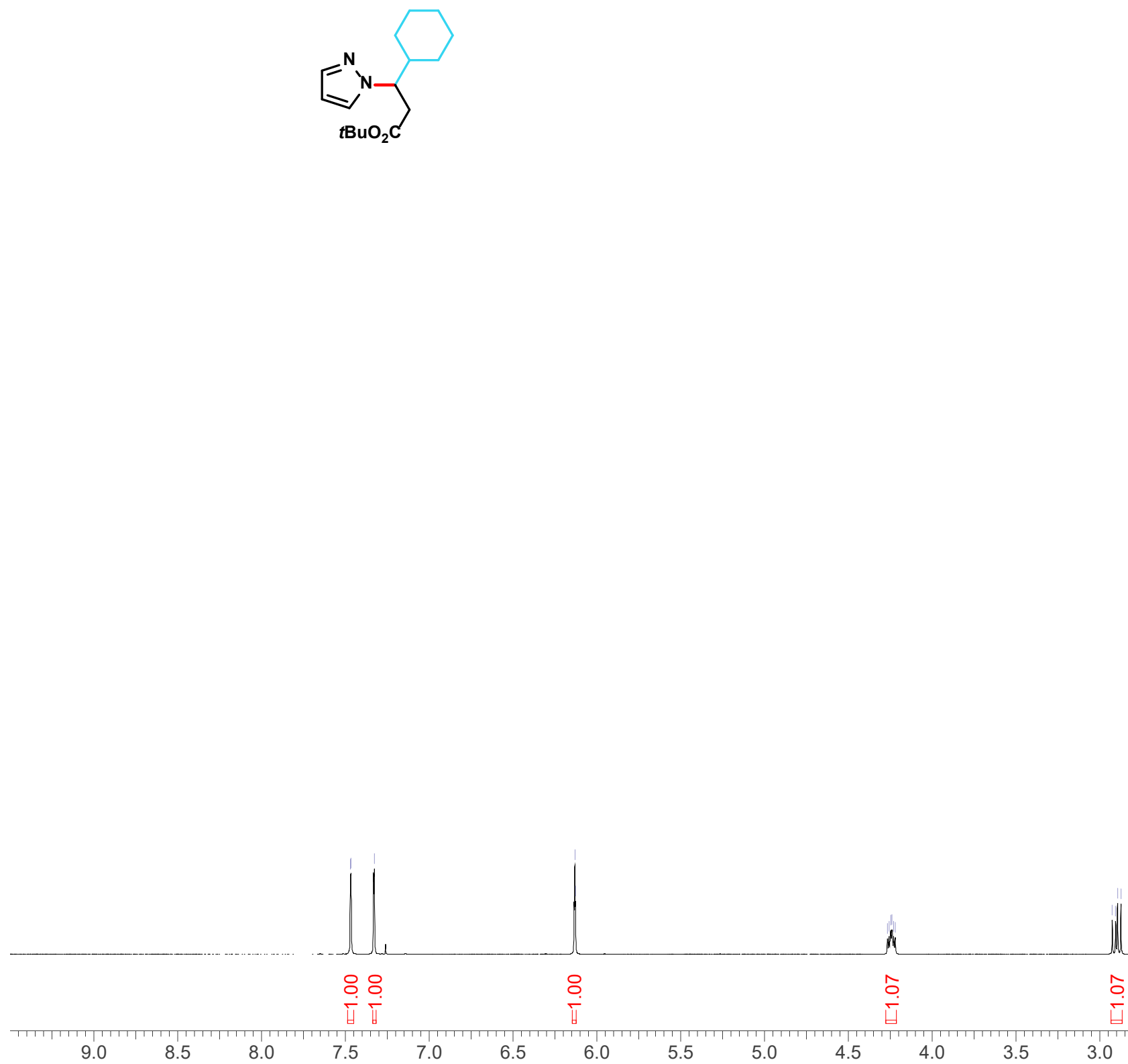
${ }^{13} \mathrm{C}\left\{{ }^{1} \mathrm{H}\right\} \mathrm{NMR}\left(126 \mathrm{MHz}, \mathrm{CDCl}_{3}\right)$ of $\mathbf{7 h}$

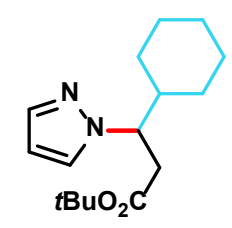


${ }^{1} \mathrm{H} \mathrm{NMR}\left(600 \mathrm{MHz}, \mathrm{CDCl}_{3}\right)$ of $7 \mathbf{i}$

ํํํ.

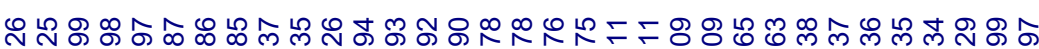

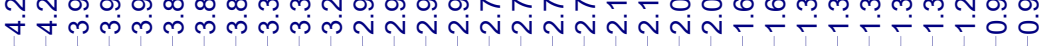

\begin{tabular}{|c|c|c|c|c|c|c|c|c|c|c|c|c|c|c|c|c|c|}
\hline & & & mo & & & 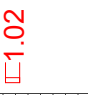 & & & & 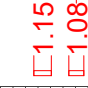 & 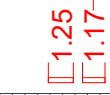 & 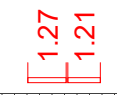 & & 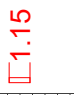 & 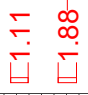 & 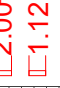 & \\
\hline 9.0 & 8.5 & 8.0 & 7.5 & 7.0 & 6.5 & 6.0 & 5.5 & 5.0 & 4.5 & 4.0 & 3.5 & 3.0 & 2.5 & 2.0 & 1.5 & 1.0 & Chemical Shift (ppm \\
\hline
\end{tabular}


${ }^{13} \mathrm{C}\left\{{ }^{1} \mathrm{H}\right\} \mathrm{NMR}\left(151 \mathrm{MHz}, \mathrm{CDCl}_{3}\right)$ of $7 \mathbf{i}$

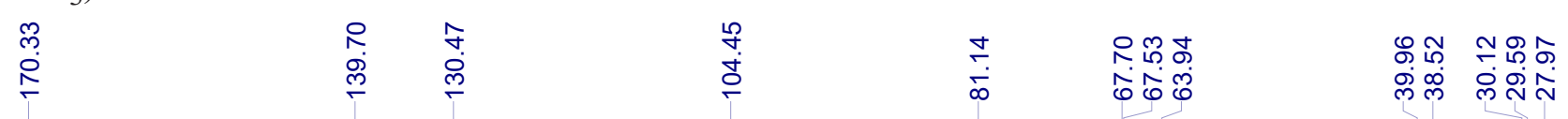

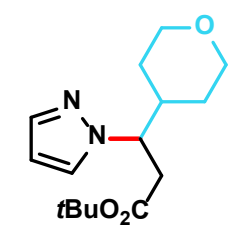


${ }^{1} \mathrm{H} \mathrm{NMR}\left(500 \mathrm{MHz}, \mathrm{CDCl}_{3}\right)$ of $7 \mathbf{j}$

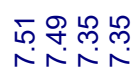
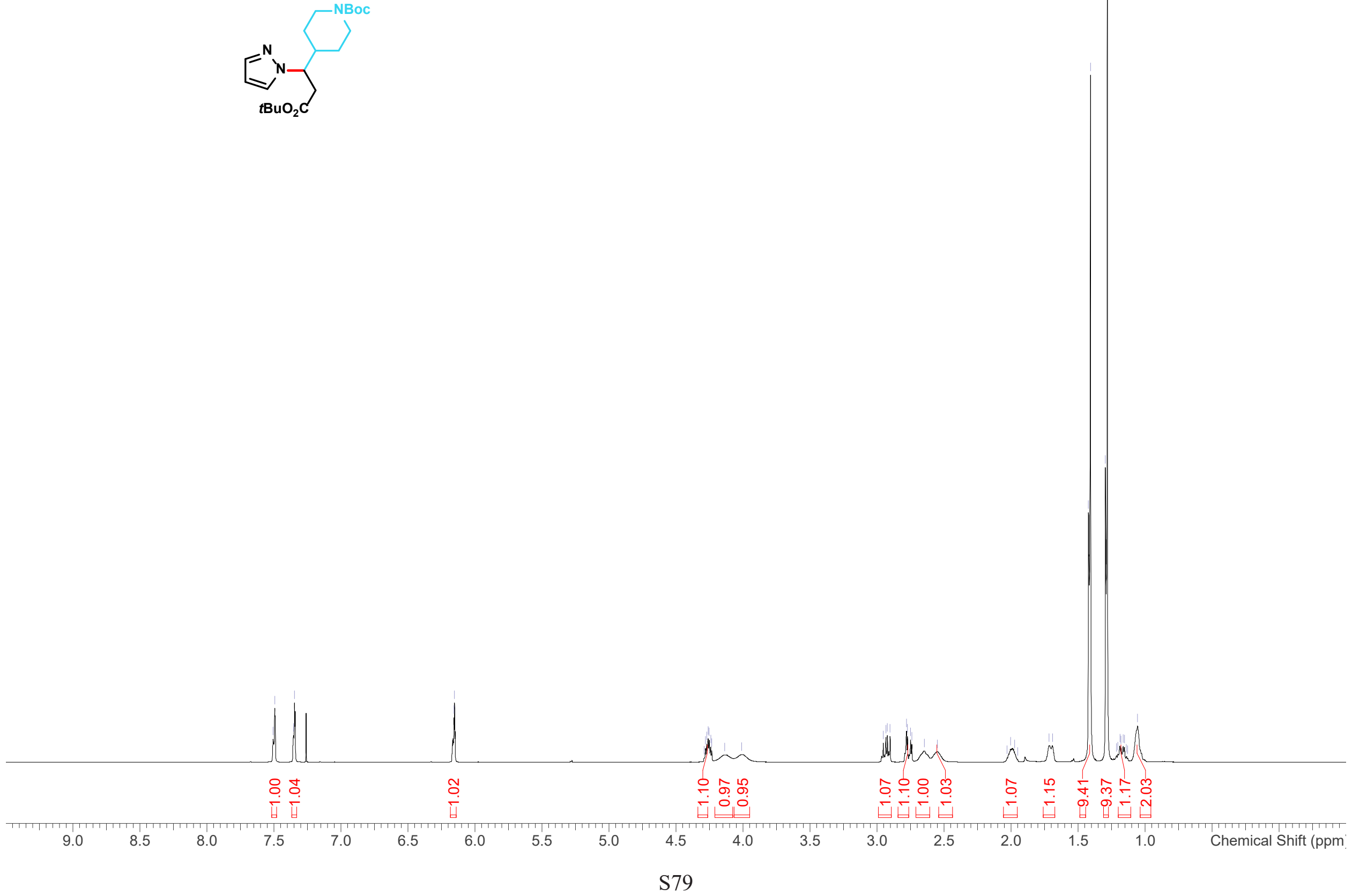
${ }^{13} \mathrm{C}\left\{{ }^{1} \mathrm{H}\right\}$ NMR $\left(126 \mathrm{MHz}, \mathrm{CDCl}_{3}\right)$ of $\mathbf{7 j}$

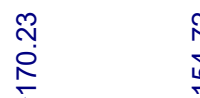

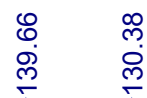

$\stackrel{\substack{0 \\ \stackrel{0}{0}}}{\stackrel{2}{+0}}$

으묨ํำ

क्ष

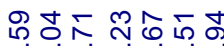

官

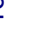

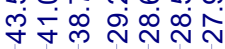

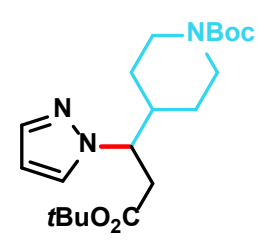




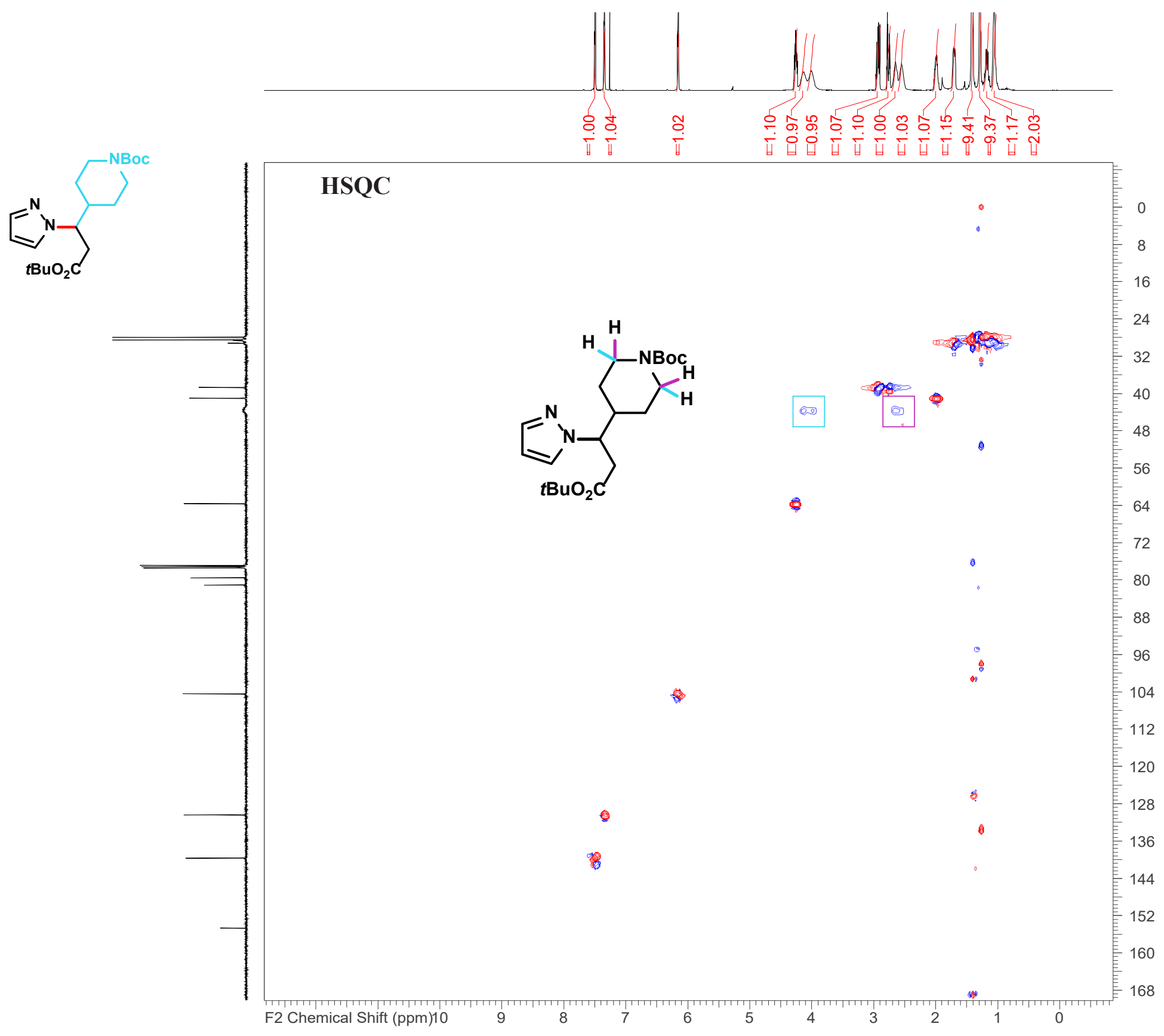


${ }^{1} \mathrm{H} \mathrm{NMR}\left(600 \mathrm{MHz}, \mathrm{CDCl}_{3}\right)$ of $\mathbf{7 k}$

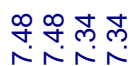

도동

음윰ㄷㄷ

字守字字

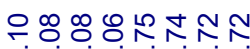

m

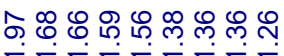
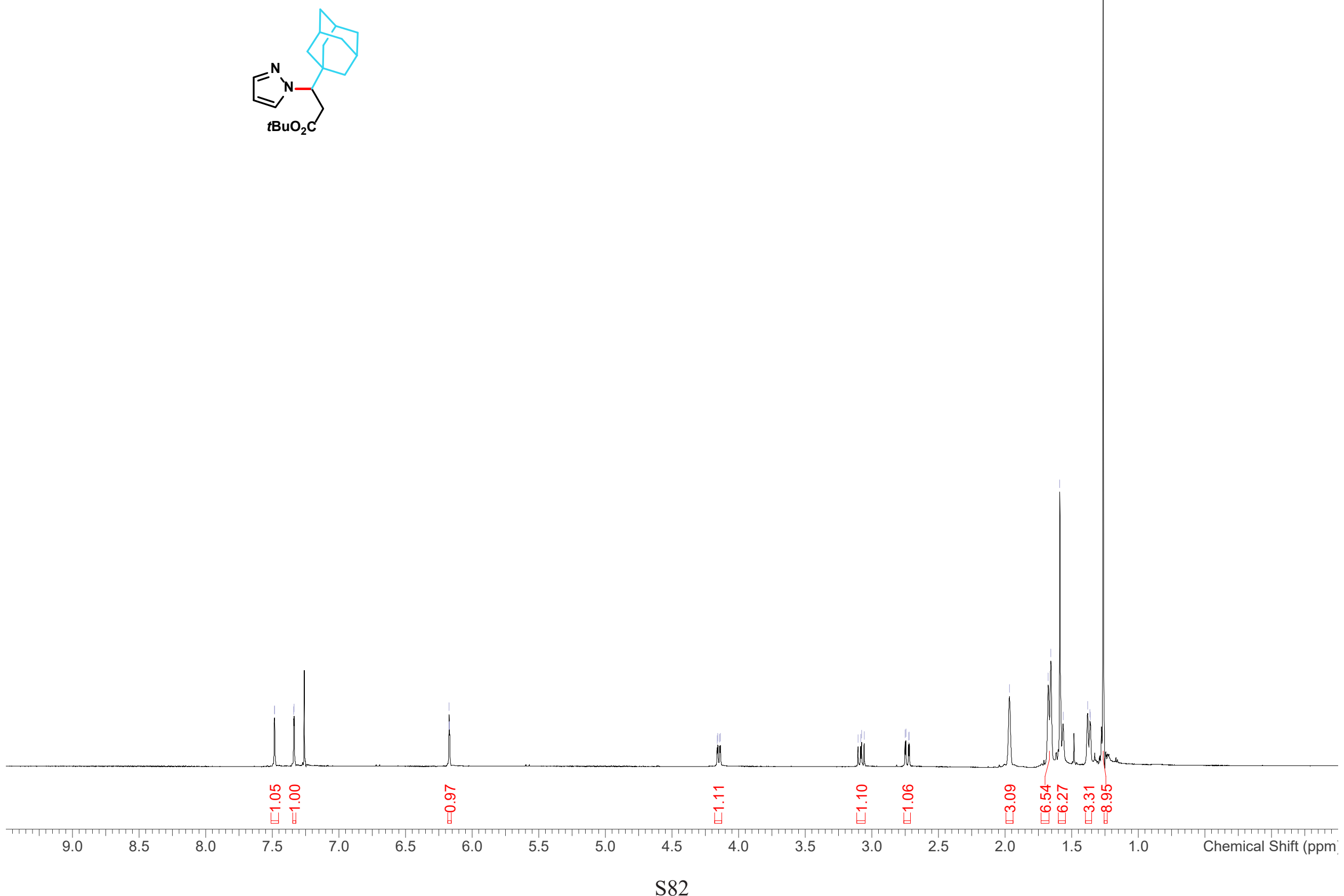
${ }^{13} \mathrm{C}\left\{{ }^{1} \mathrm{H}\right\} \mathrm{NMR}\left(151 \mathrm{MHz}, \mathrm{CDCl}_{3}\right)$ of $\mathbf{7 k}$

$$
\text { ( }
$$

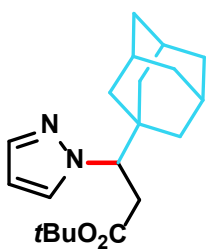

$\underset{\substack{\infty \\ \stackrel{\infty}{N}}}{\stackrel{-}{m}}$

¿

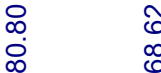

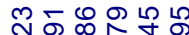


${ }^{1} \mathrm{H} \mathrm{NMR}\left(500 \mathrm{MHz}, \mathrm{CDCl}_{3}\right)$ of $7 \mathbf{l}$

垫

$\stackrel{ }{0} \frac{0}{60}$

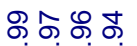

लंल लिं

$\stackrel{\stackrel{\llcorner}{\sim ⿻}}{\stackrel{\infty}{N}}$

包

$$
\underbrace{\mathrm{N}-\underbrace{\mathrm{Me}}}_{\mathrm{EtO}_{2} \mathrm{C}}
$$

\begin{tabular}{|c|c|c|c|c|c|c|c|c|c|c|c|c|c|c|c|c|c|}
\hline & & & $\stackrel{\stackrel{\infty}{\leftarrow}}{\stackrel{\square}{4}}$ & & & & & & & $\stackrel{\bar{i}}{\tilde{U}}$ & & & & & & 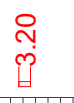 & \\
\hline 9.0 & 8.5 & 8.0 & 7.5 & 7.0 & 6.5 & 6.0 & 5.5 & 5.0 & 4.5 & 4.0 & 3.5 & 3.0 & 2.5 & 2.0 & 1.5 & 1.0 & Chemical Shift (ppm \\
\hline
\end{tabular}


${ }^{13} \mathrm{C}\left\{{ }^{1} \mathrm{H}\right\} \mathrm{NMR}\left(126 \mathrm{MHz}, \mathrm{CDCl}_{3}\right)$ of $7 \mathbf{l}$

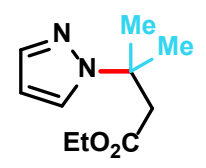


${ }^{1} \mathrm{H}$ NMR $\left(500 \mathrm{MHz}, \mathrm{CDCl}_{3}\right)$ of $\mathbf{7 m}$

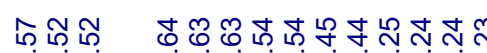
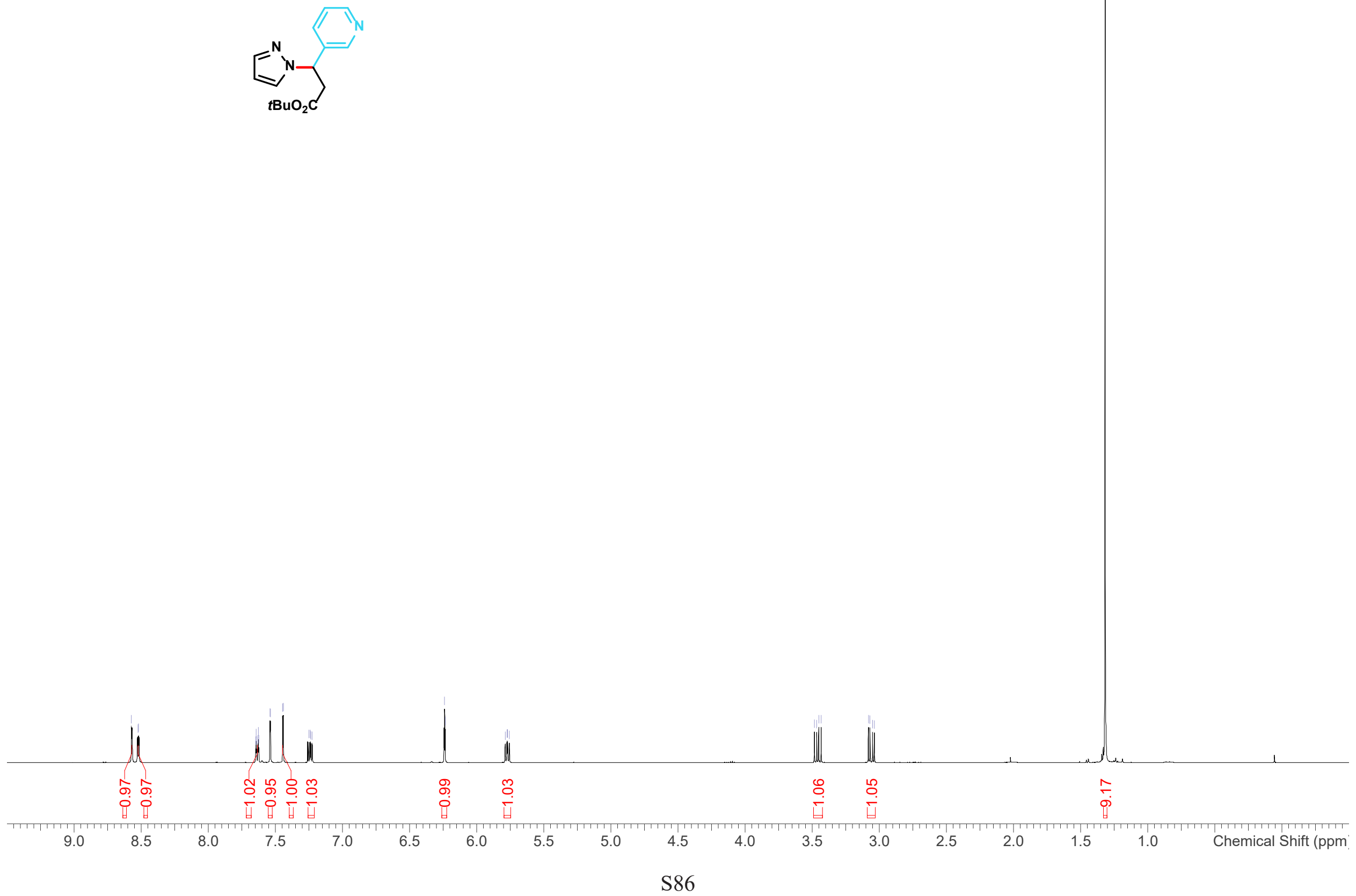
${ }^{13} \mathrm{C}\left\{{ }^{1} \mathrm{H}\right\} \mathrm{NMR}\left(126 \mathrm{MHz}, \mathrm{CDCl}_{3}\right)$ of $7 \mathbf{m}$

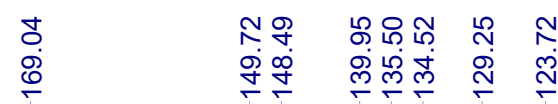

నू

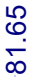

ڤิ)

$\bar{\zeta}$

ลุ่

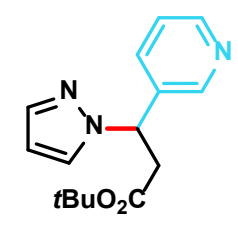

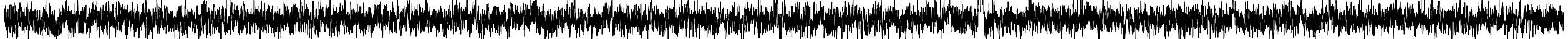

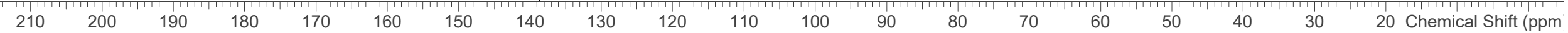


${ }^{1} \mathrm{H} \mathrm{NMR}\left(600 \mathrm{MHz}, \mathrm{CDCl}_{3}\right)$ of $\mathbf{7 n}$

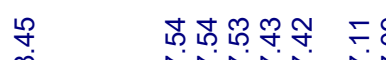

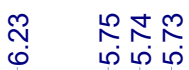

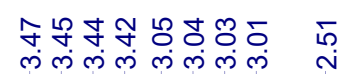

$\stackrel{\sim}{\sim}$
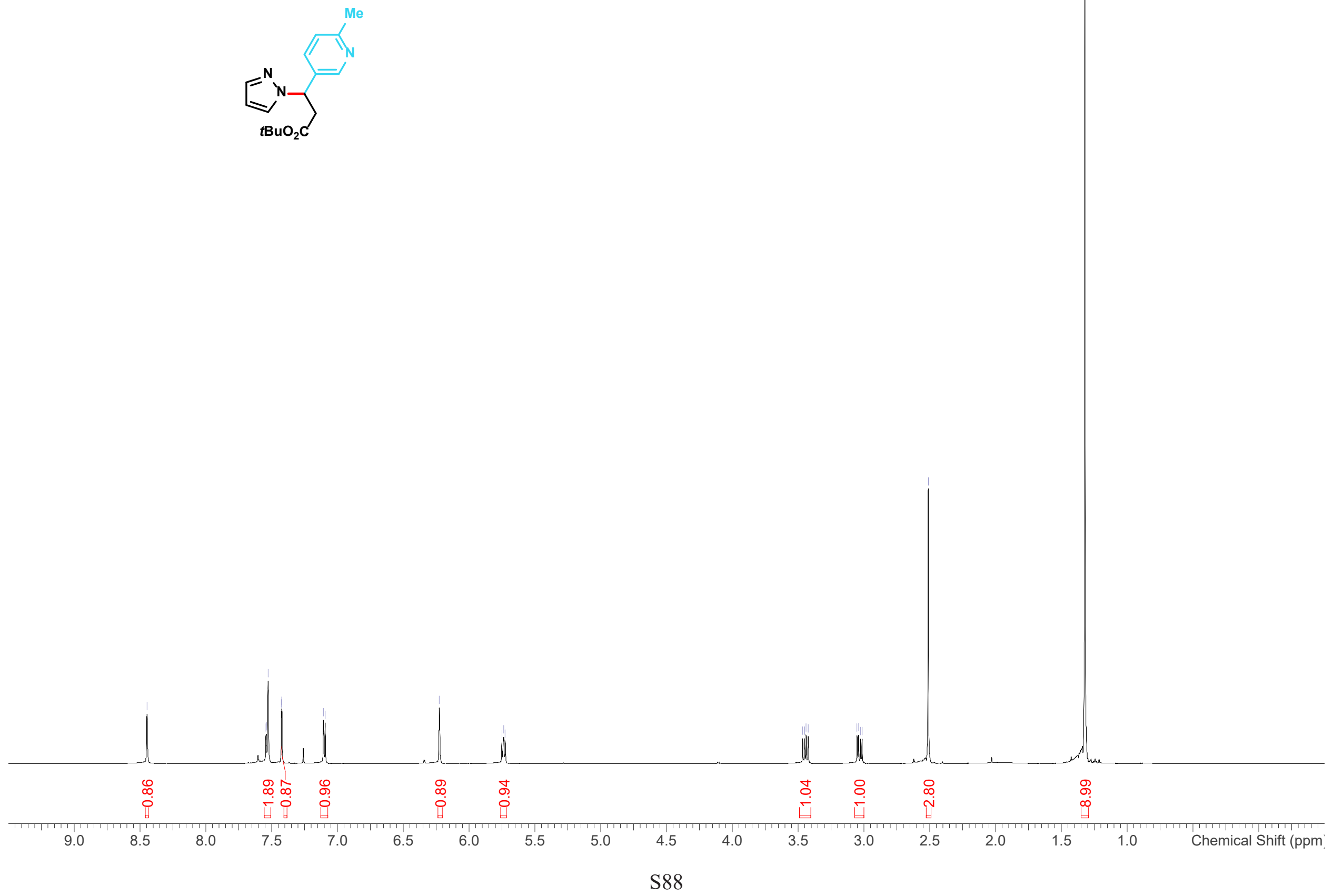
${ }^{13} \mathrm{C}\left\{{ }^{1} \mathrm{H}\right\} \mathrm{NMR}\left(126 \mathrm{MHz}, \mathrm{CDCl}_{3}\right)$ of $\mathbf{7 n}$

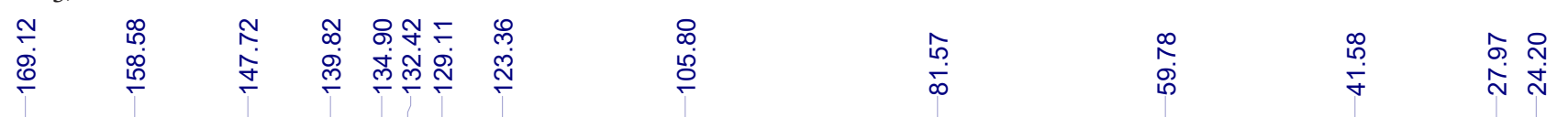

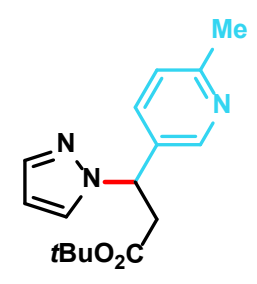

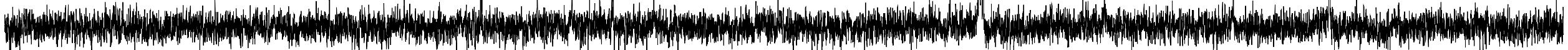

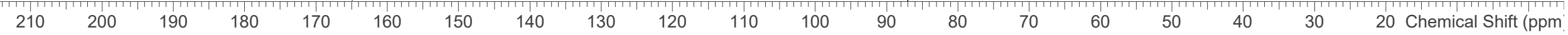


${ }^{1} \mathrm{H} \mathrm{NMR}\left(600 \mathrm{MHz}, \mathrm{CDCl}_{3}\right)$ of $7 \mathbf{o}$

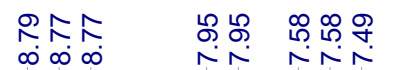

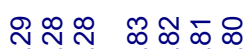



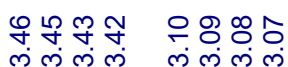

$\stackrel{\text { m. }}{\longrightarrow}$
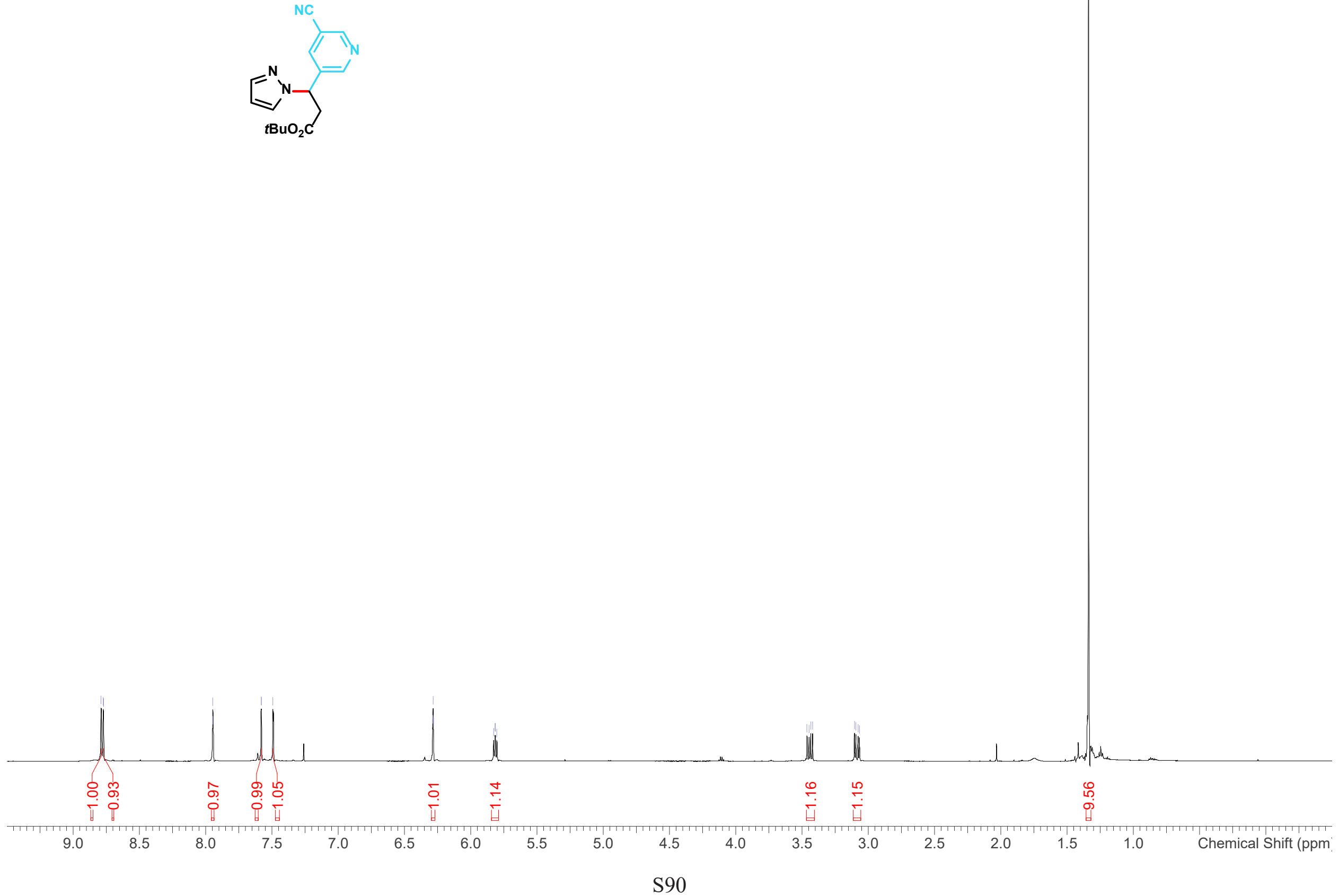
${ }^{13} \mathrm{C}\left\{{ }^{1} \mathrm{H}\right\} \mathrm{NMR}\left(151 \mathrm{MHz}, \mathrm{CDCl}_{3}\right)$ of $7 \mathbf{o}$

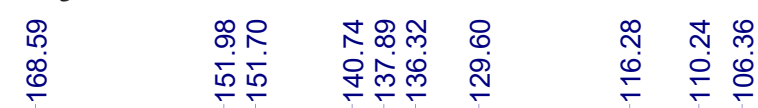

$\underset{\infty}{\stackrel{\infty}{\sim}}$

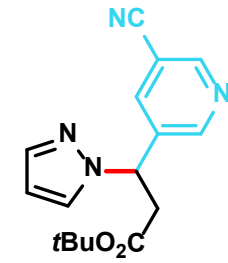


${ }^{1} \mathrm{H} \mathrm{NMR}\left(600 \mathrm{MHz}, \mathrm{CDCl}_{3}\right)$ of $7 \mathbf{p}$

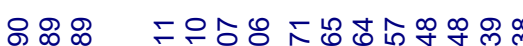

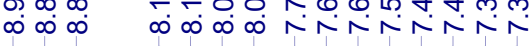

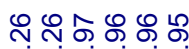

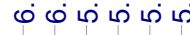

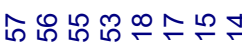

लंखिय लं लं लंख

$\stackrel{\text { న }}{-}$
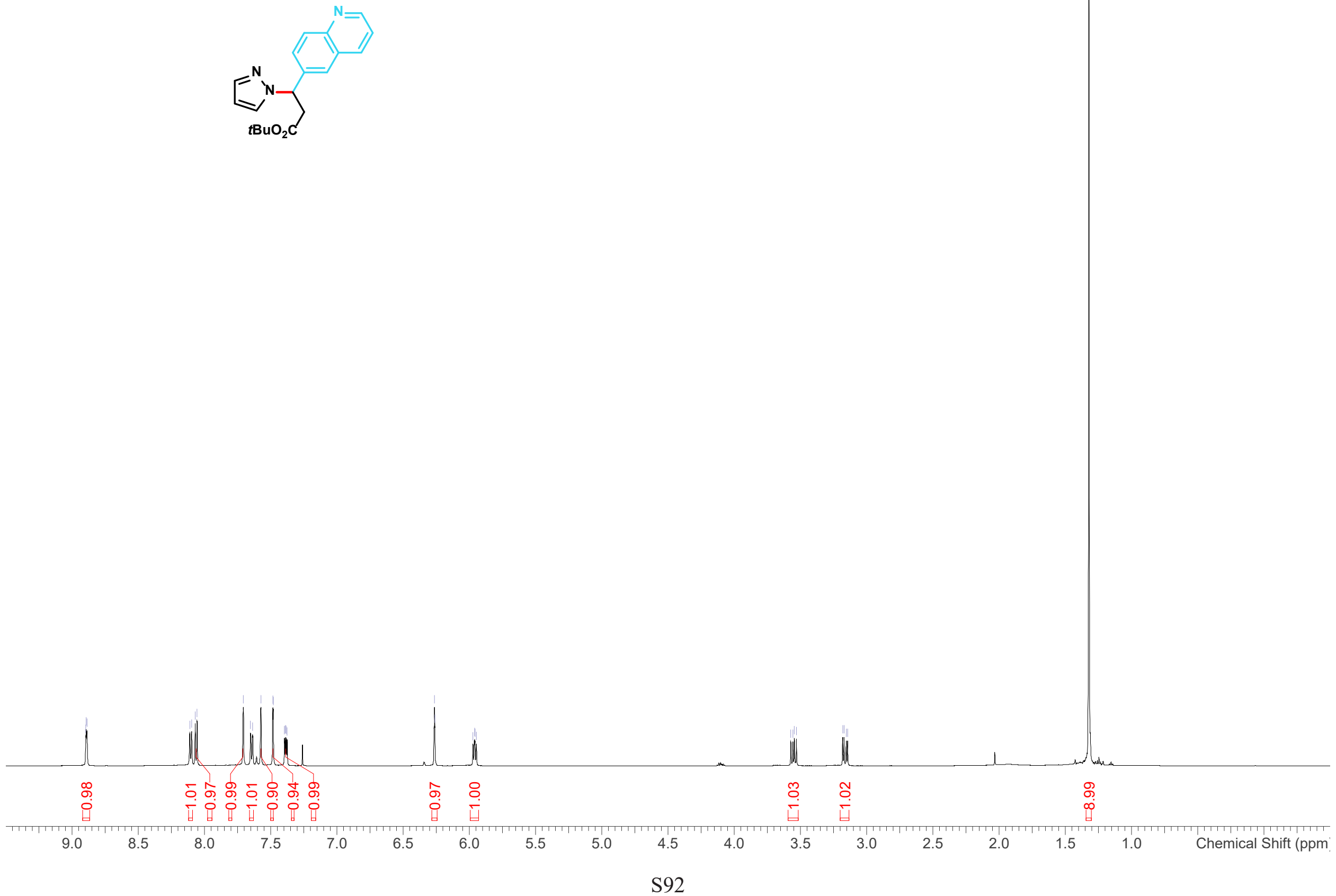
${ }^{13} \mathrm{C}\left\{{ }^{1} \mathrm{H}\right\} \mathrm{NMR}\left(151 \mathrm{MHz}, \mathrm{CDCl}_{3}\right)$ of $\mathbf{7 p}$

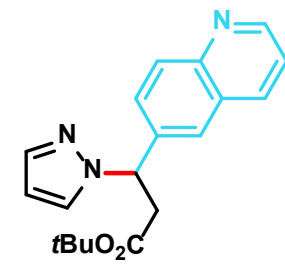


${ }^{1} \mathrm{H} \mathrm{NMR}\left(500 \mathrm{MHz}, \mathrm{CDCl}_{3}\right)$ of $\mathbf{7 q}$

8

ing

ดु

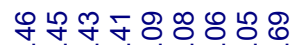

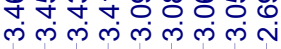
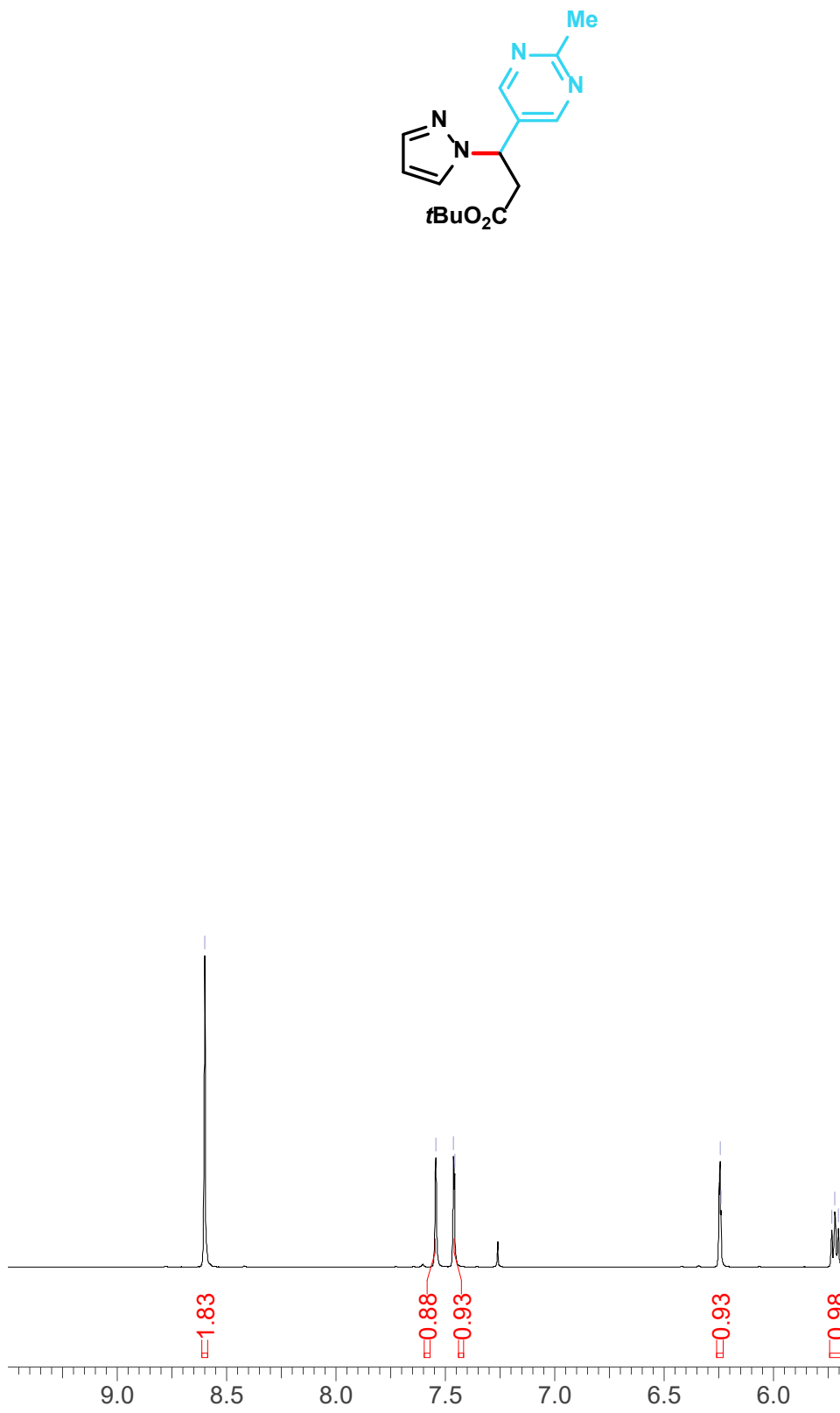

$6.0 \quad 5.5$

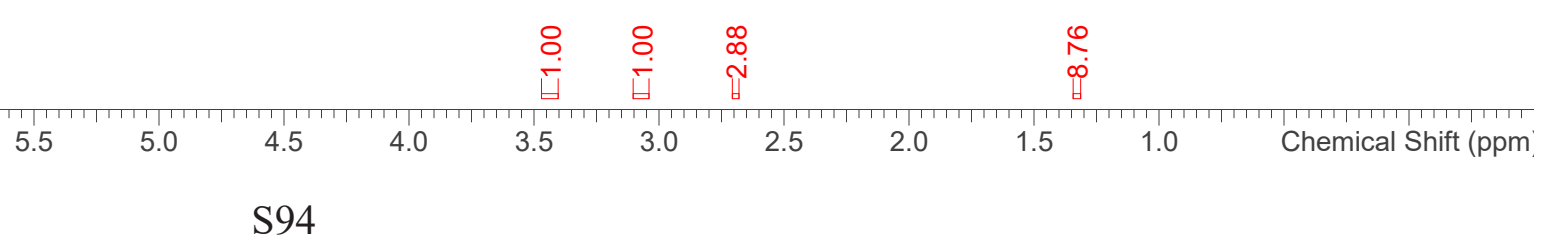


${ }^{13} \mathrm{C}\left\{{ }^{1} \mathrm{H}\right\} \mathrm{NMR}\left(126 \mathrm{MHz}, \mathrm{CDCl}_{3}\right)$ of $\mathbf{7 q}$

잉

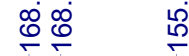

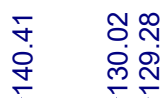

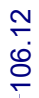

$\frac{\hat{s}}{\infty}$

$\underset{\substack{0 \\ \infty}}{\stackrel{0}{\infty}}$

กุ.

ลุ

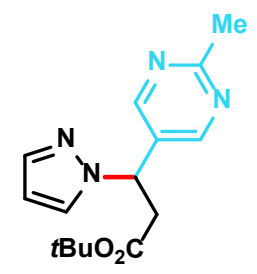

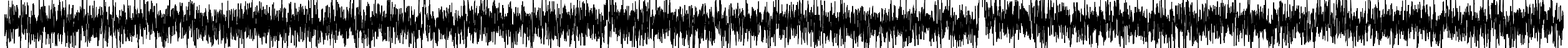


${ }^{1} \mathrm{H} \mathrm{NMR}\left(600 \mathrm{MHz}, \mathrm{CDCl}_{3}\right)$ of $\mathbf{7 r}$

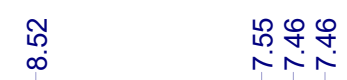

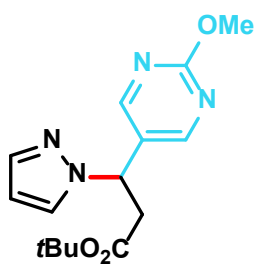

\begin{tabular}{|c|c|c|c|c|c|c|c|c|c|c|c|c|c|c|c|c|c|}
\hline & $\stackrel{8}{\check{\leftarrow}}$ & & $\begin{array}{ll}1 & 1 \\
\infty & 0 \\
0 & 0 \\
ن & 0 \\
\dot{y} & 0\end{array}$ & & & & $\begin{array}{l}8 \\
\stackrel{\circ}{\circ} \\
\end{array}$ & & & $\stackrel{\mathcal{N}}{\stackrel{N}{\sim}}$ & $\stackrel{8}{\stackrel{5}{\leftarrow}}$ & $\stackrel{8}{\stackrel{5}{5}}$ & & & & & \\
\hline 9.0 & 8.5 & 8.0 & 7.5 & 7.0 & 6.5 & 6.0 & 5.5 & 5.0 & 4.5 & 4.0 & 3.5 & 3.0 & 2.5 & 2.0 & 1.5 & 1.0 & Chemical Shift (ppm \\
\hline
\end{tabular}


${ }^{13} \mathrm{C}\left\{{ }^{1} \mathrm{H}\right\}$ NMR $\left(151 \mathrm{MHz}, \mathrm{CDCl}_{3}\right)$ of $7 \mathbf{r}$

ธ。 คำ $\bar{m}$

$\stackrel{\infty}{\circ} \stackrel{\infty}{\stackrel{0}{\circ}}$

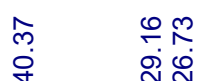

$\varnothing$
$\stackrel{\circ}{\circ}$

$\stackrel{8}{\infty}$

N

ถิ่

$\stackrel{5}{\stackrel{5}{+}}$

$\stackrel{\substack{0 \\ \text { o }}}{\infty}$

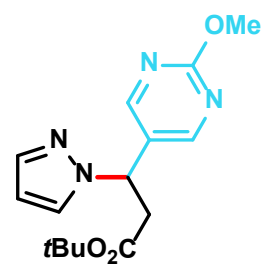


${ }^{1} \mathrm{H} \mathrm{NMR}\left(600 \mathrm{MHz}, \mathrm{CDCl}_{3}\right)$ of $\mathbf{7 s}$

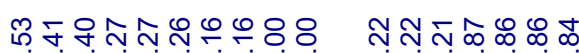

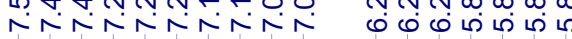

우욖

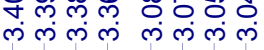
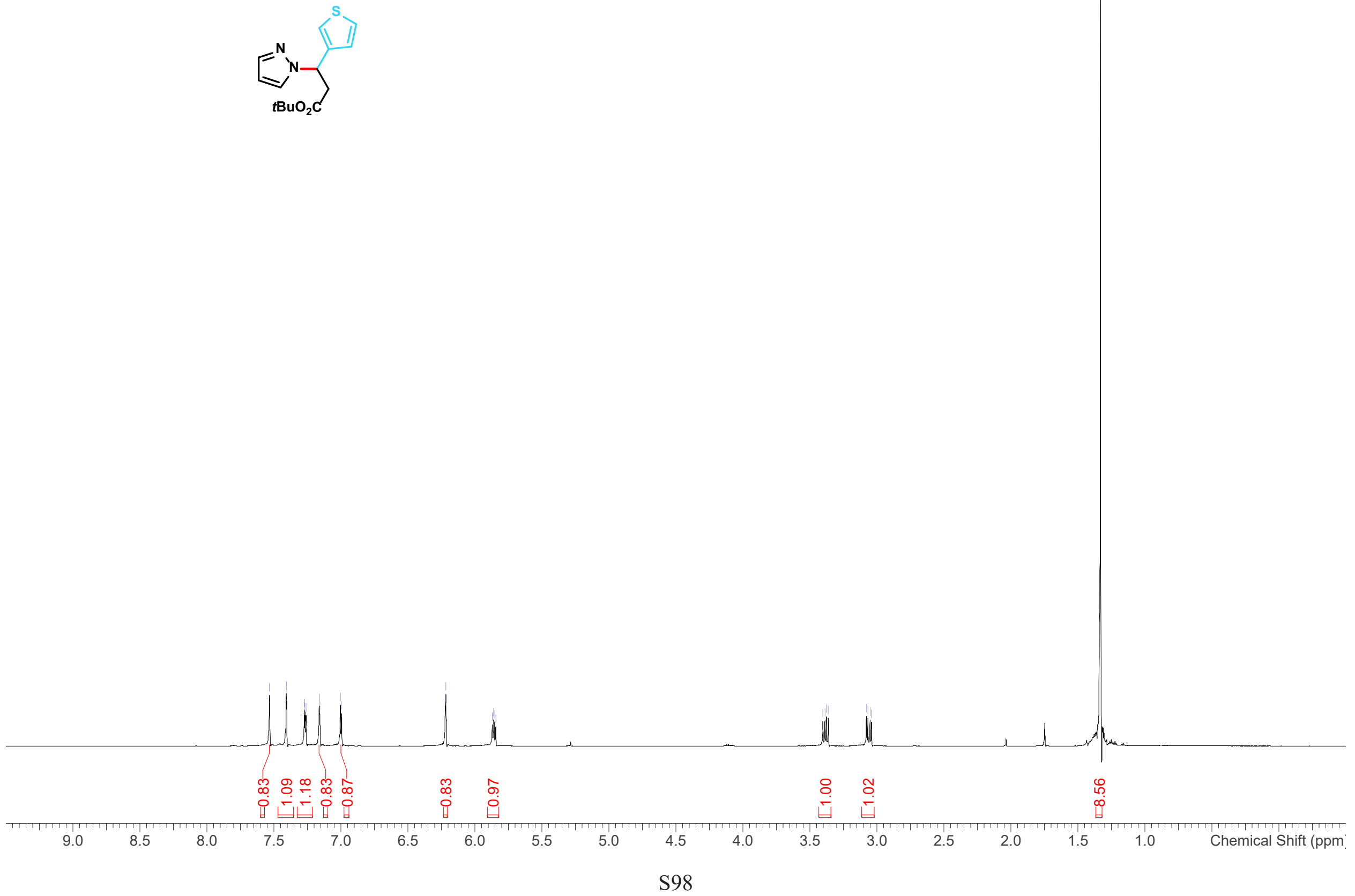
${ }^{13} \mathrm{C}\left\{{ }^{1} \mathrm{H}\right\} \mathrm{NMR}\left(151 \mathrm{MHz}, \mathrm{CDCl}_{3}\right)$ of $\mathbf{7 s}$

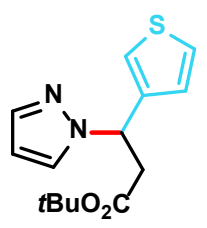


${ }^{1} \mathrm{H} \mathrm{NMR}\left(600 \mathrm{MHz}, \mathrm{CDCl}_{3}\right)$ of $\mathbf{7 t}$
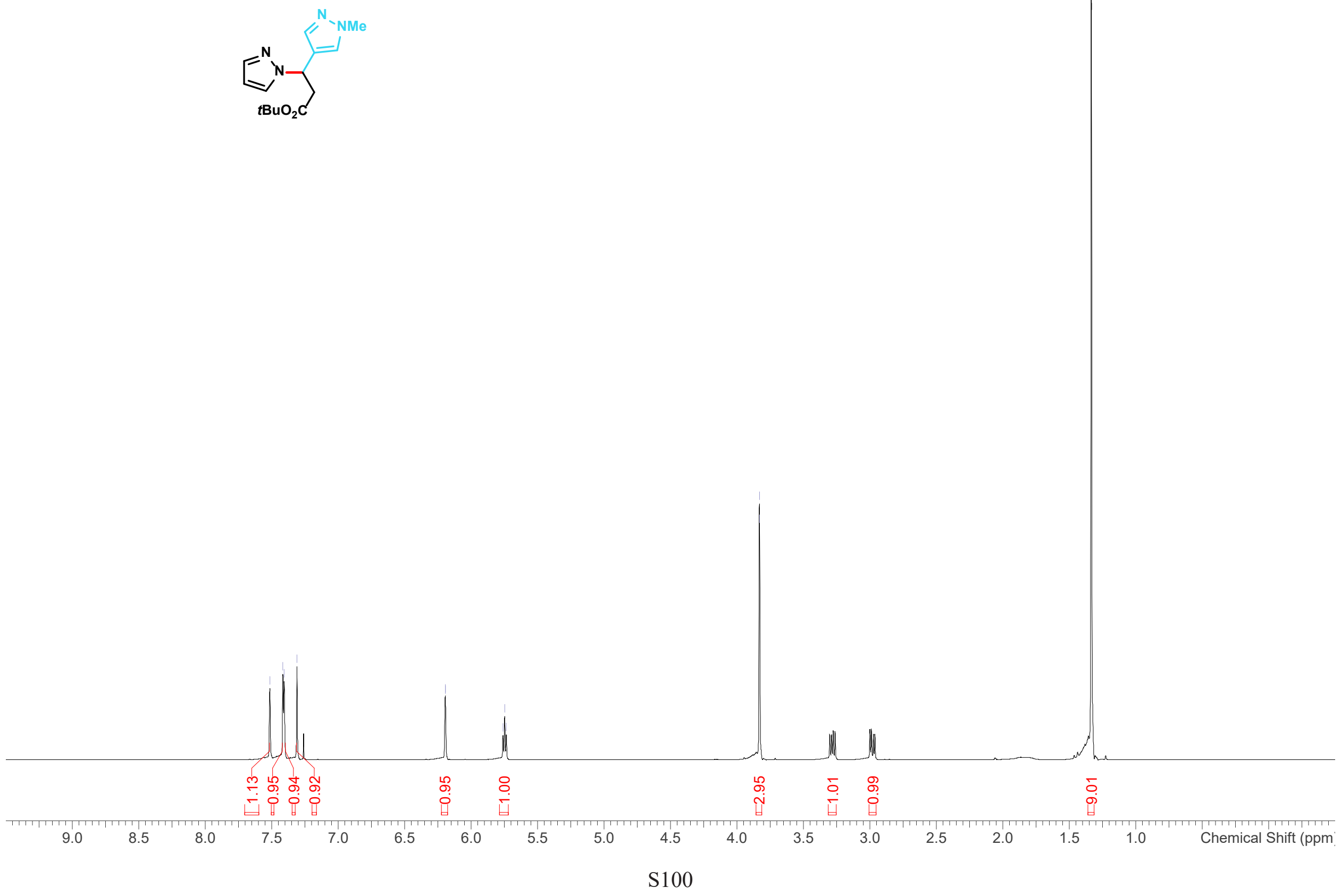
${ }^{13} \mathrm{C}\left\{{ }^{1} \mathrm{H}\right\} \mathrm{NMR}\left(151 \mathrm{MHz}, \mathrm{CDCl}_{3}\right)$ of $7 \mathbf{t}$

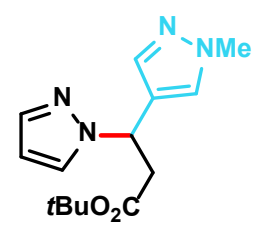


${ }^{1} \mathrm{H}$ NMR $\left(500 \mathrm{MHz}, \mathrm{CDCl}_{3}\right)$ of $\mathbf{8 a}$

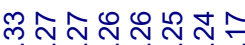

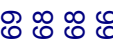

以่乩的

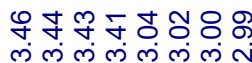

กับ
กับ

$\stackrel{m}{\sim}$

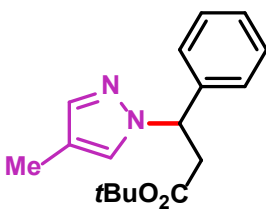

\begin{tabular}{|c|c|c|c|c|c|c|c|c|c|c|c|c|c|c|c|c|c|}
\hline & & & & & & & & & & & $\begin{array}{l}8 \\
\dot{\square}\end{array}$ & 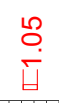 & & $\stackrel{\sim}{\stackrel{D}{U}}$ & & & \\
\hline 9.0 & 8.5 & 8.0 & 7.5 & 7.0 & 6.5 & 6.0 & 5.5 & 5.0 & 4.5 & 4.0 & 3.5 & 3.0 & 2.5 & 2.0 & 1.5 & 1.0 & Chemical Shift (ppm \\
\hline
\end{tabular}


${ }^{13} \mathrm{C}\left\{{ }^{1} \mathrm{H}\right\} \mathrm{NMR}\left(126 \mathrm{MHz}, \mathrm{CDCl}_{3}\right)$ of $\mathbf{8 a}$

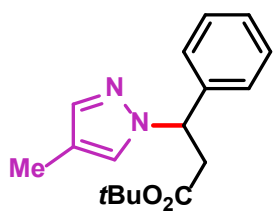


${ }^{1} \mathrm{H}$ NMR $\left(600 \mathrm{MHz}, \mathrm{CDCl}_{3}\right)$ of $\mathbf{8 b}$

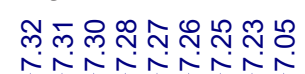

ชูญธูธ

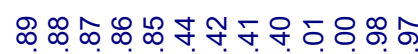

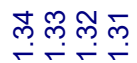
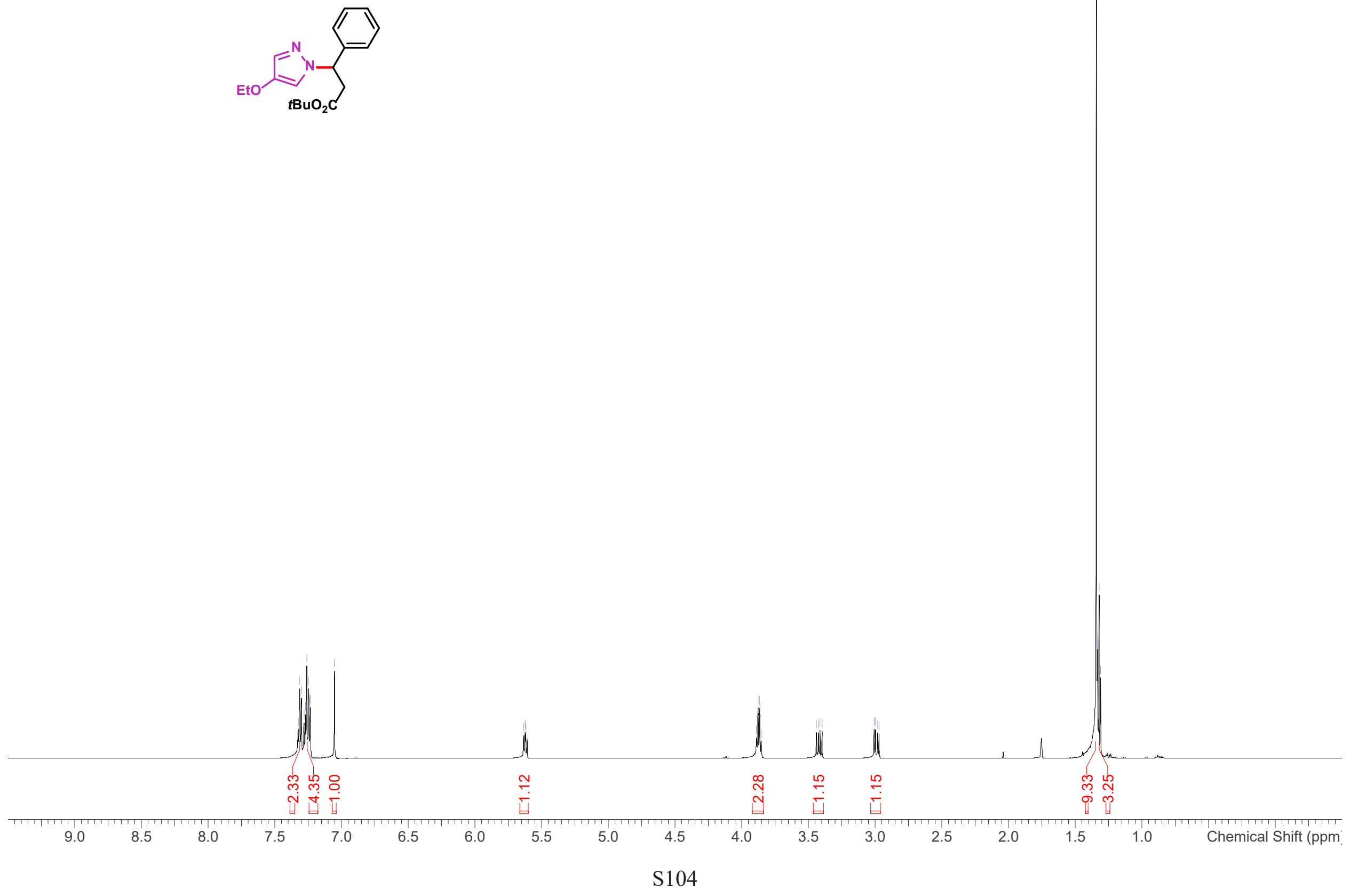
${ }^{13} \mathrm{C}\left\{{ }^{1} \mathrm{H}\right\}$ NMR $\left(151 \mathrm{MHz}, \mathrm{CDCl}_{3}\right)$ of $\mathbf{8 b}$

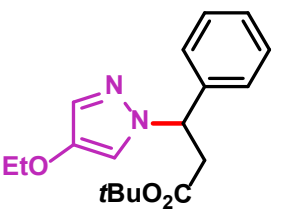


${ }^{1} \mathrm{H} \mathrm{NMR}\left(500 \mathrm{MHz}, \mathrm{CDCl}_{3}\right)$ of $\mathbf{8 c}$
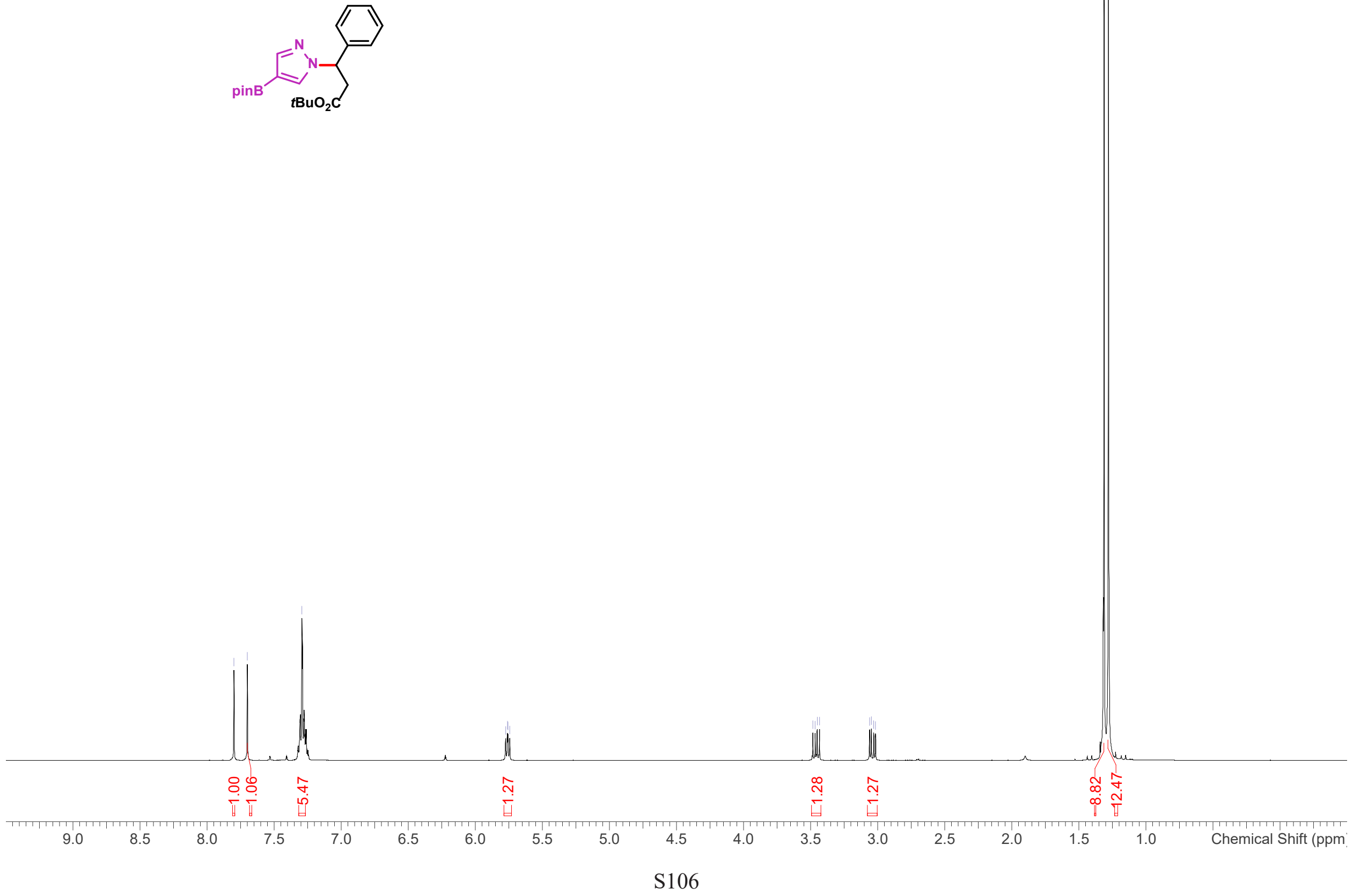
${ }^{13} \mathrm{C}\left\{{ }^{1} \mathrm{H}\right\} \mathrm{NMR}\left(126 \mathrm{MHz}, \mathrm{CDCl}_{3}\right)$ of $\mathbf{8 c}$ 
${ }^{1} \mathrm{H}-{ }^{13} \mathrm{C} \mathrm{HMBC} \mathrm{NMR}\left(\mathrm{CDCl}_{3}\right)$ of $\mathbf{8 c}$

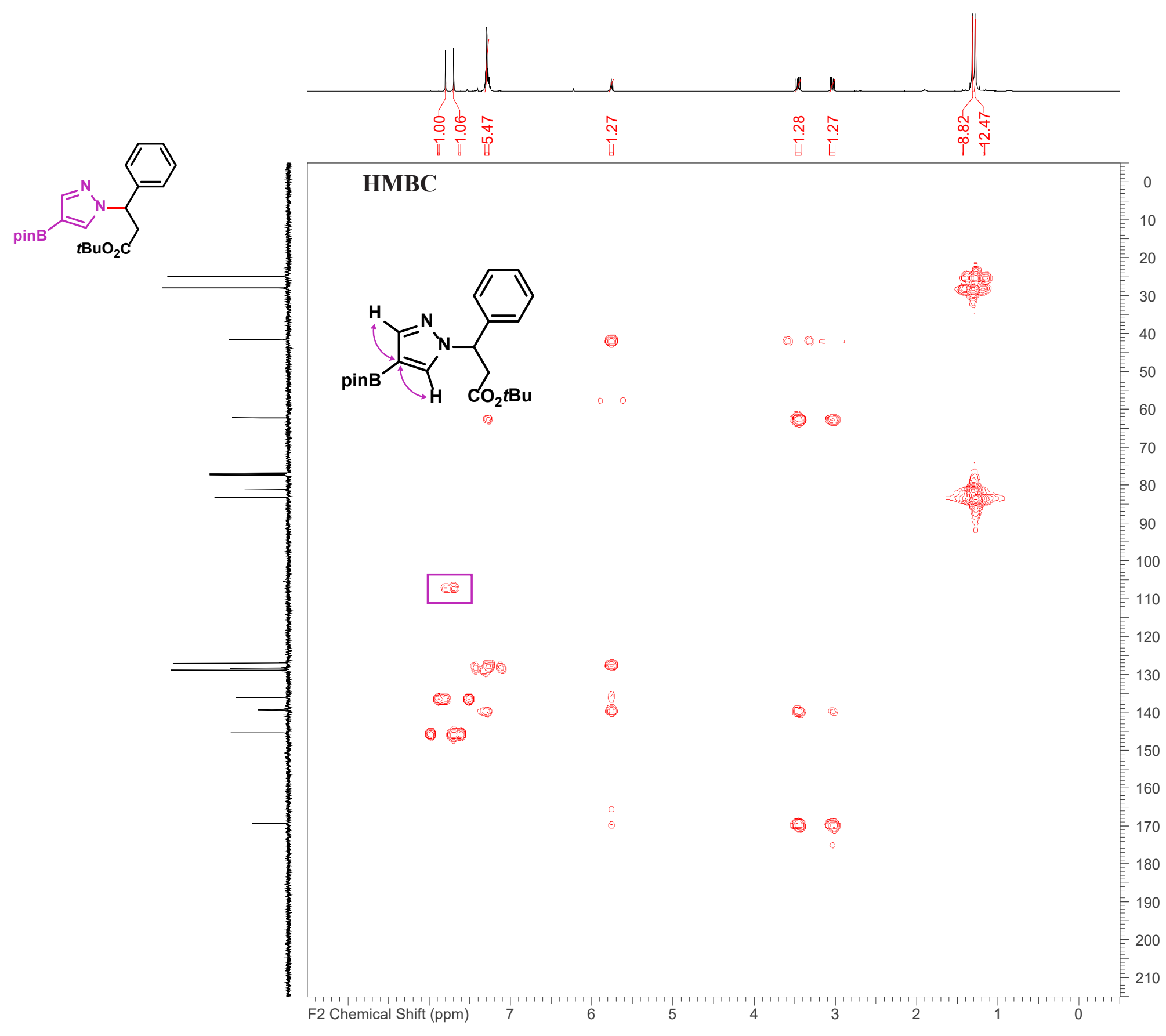




\section{${ }^{1} \mathrm{H}$ NMR $\left(500 \mathrm{MHz}, \mathrm{CDCl}_{3}\right)$ of $\mathbf{8 d}$}

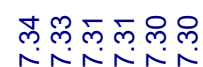

$\forall \cong \quad \widehat{~}$

б.

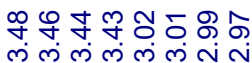

$\stackrel{+}{\stackrel{5}{*}}$
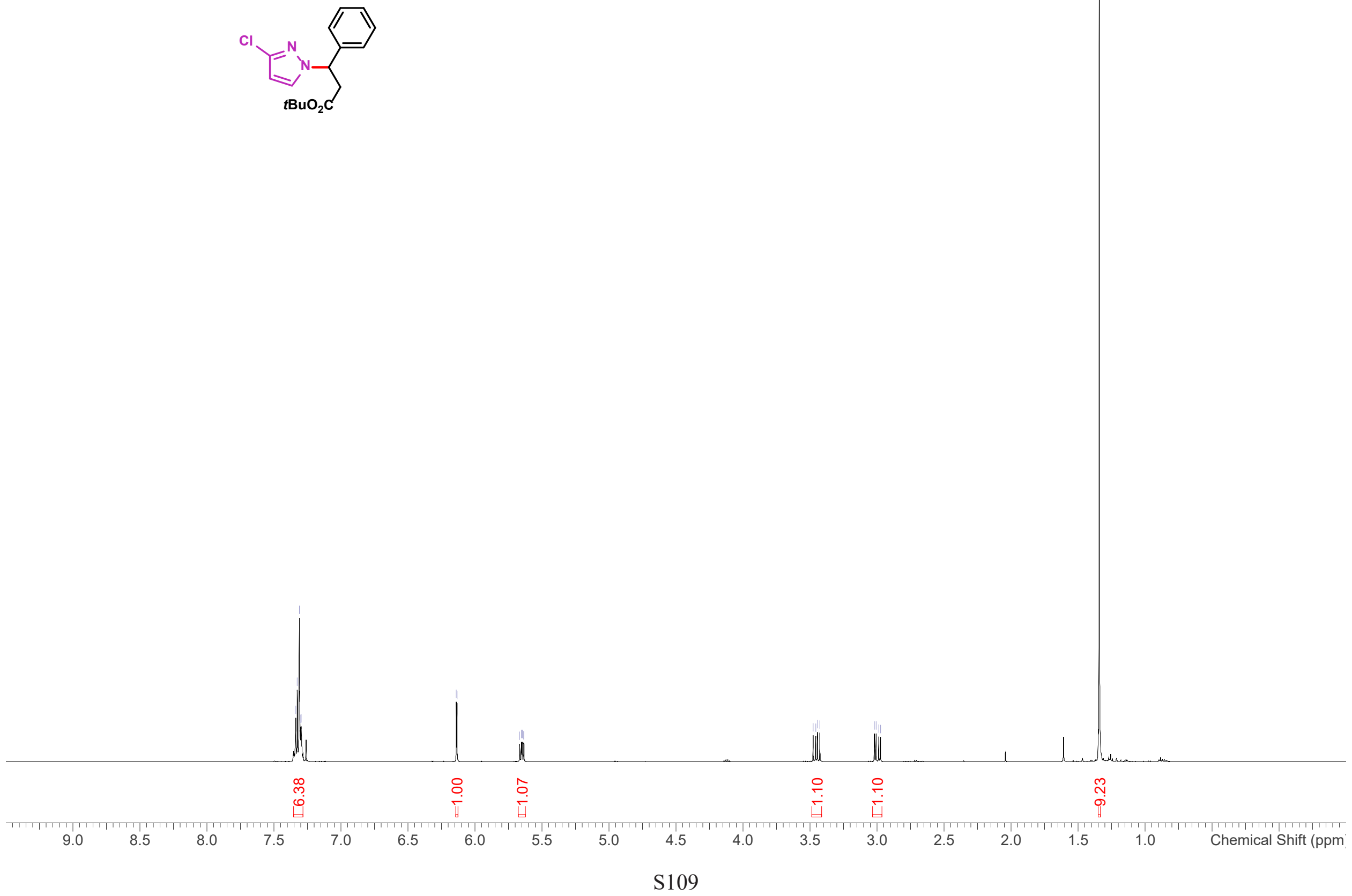
${ }^{13} \mathrm{C}\left\{{ }^{1} \mathrm{H}\right\} \mathrm{NMR}\left(126 \mathrm{MHz}, \mathrm{CDCl}_{3}\right)$ of $\mathbf{8 d}$

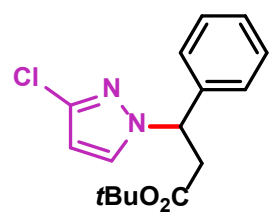


${ }^{1} \mathrm{H}-{ }^{13} \mathrm{C} \mathrm{HMBC} \mathrm{NMR}\left(\mathrm{CDCl}_{3}\right)$ of $\mathbf{8 d}$

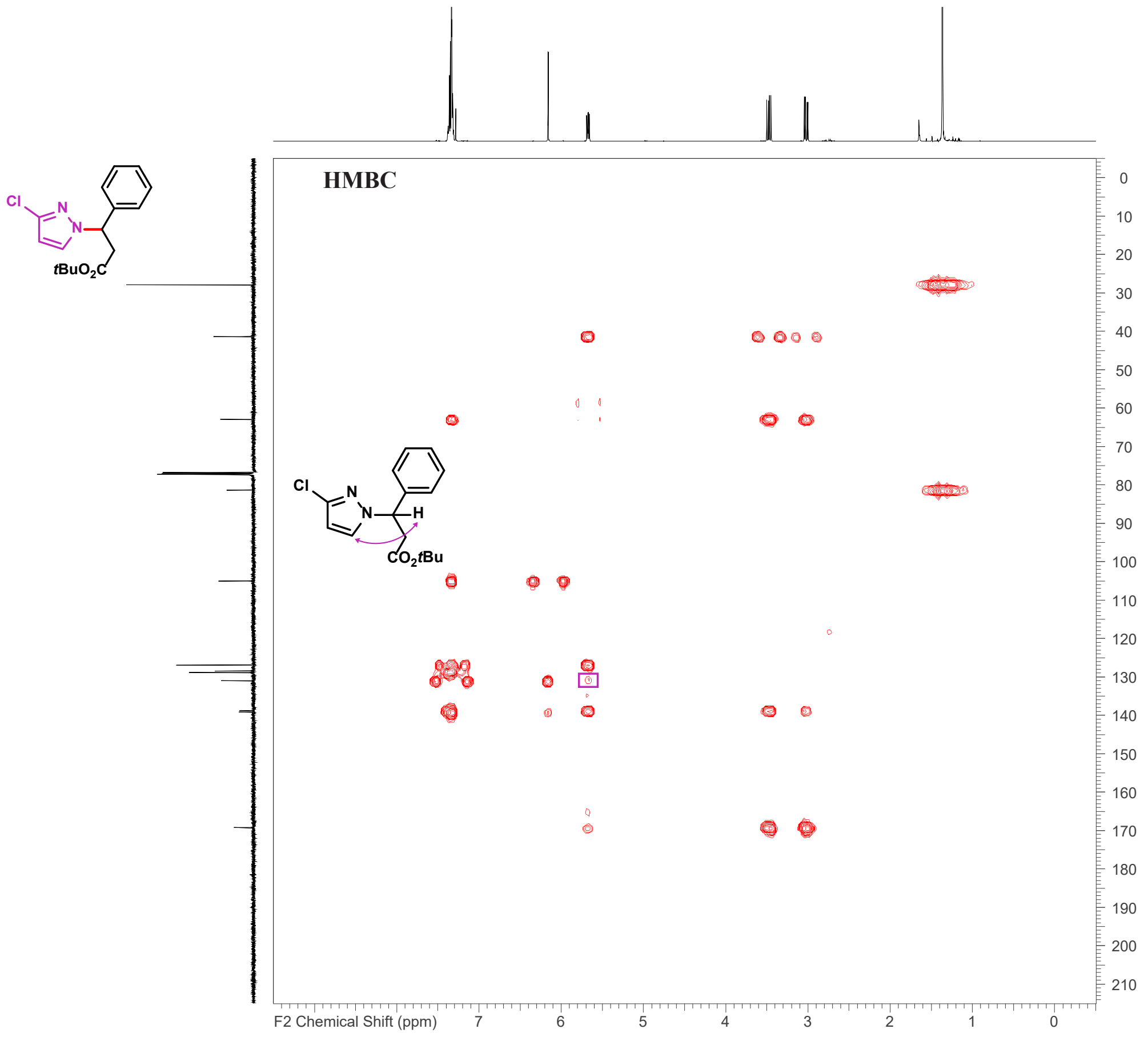


${ }^{1} \mathrm{H} \mathrm{NMR}\left(500 \mathrm{MHz}, \mathrm{CDCl}_{3}\right)$ of $\mathbf{8 e}$

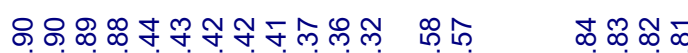

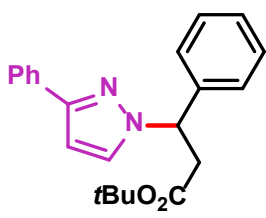

\begin{tabular}{|c|c|c|c|c|c|c|c|c|c|c|c|c|c|c|c|c|c|}
\hline & & $\begin{array}{l}\stackrel{\leftrightarrow}{\dot{y}} \\
\end{array}$ & 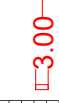 & & $\begin{array}{l}\hat{o} \\
\text { Oे }\end{array}$ & & & & & & : & $\begin{array}{l}8 \\
\dot{\square}\end{array}$ & & & 8 & & \\
\hline 9.0 & 8.5 & 8.0 & 7.5 & 7.0 & 6.5 & 6.0 & 5.5 & 5.0 & 4.5 & 4.0 & 3.5 & 3.0 & 2.5 & 2.0 & 1.5 & 1.0 & Chemical Shift (ppm \\
\hline
\end{tabular}


${ }^{13} \mathrm{C} \mathrm{NMR}\left\{{ }^{1} \mathrm{H}\right\}\left(126 \mathrm{MHz}, \mathrm{CDCl}_{3}\right)$ of $\mathbf{8 e}$

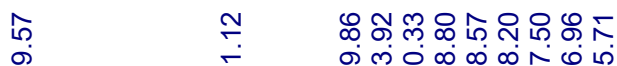

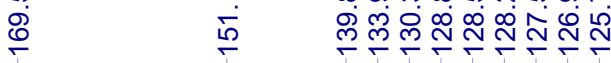

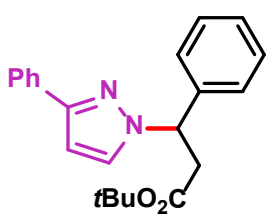

ธิธ

$\underset{\infty}{\stackrel{\infty}{\sim}}$

ปั่

$\stackrel{8}{\stackrel{+}{+}}$

$\stackrel{\widehat{s}}{\text { N }}$ 
${ }^{1} \mathrm{H} \mathrm{NMR}\left(600 \mathrm{MHz}, \mathrm{CDCl}_{3}\right)$ of $\mathbf{8 f}$

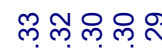

ヘヘNヘ

อัำกㅇ

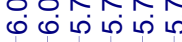

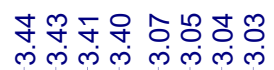

N
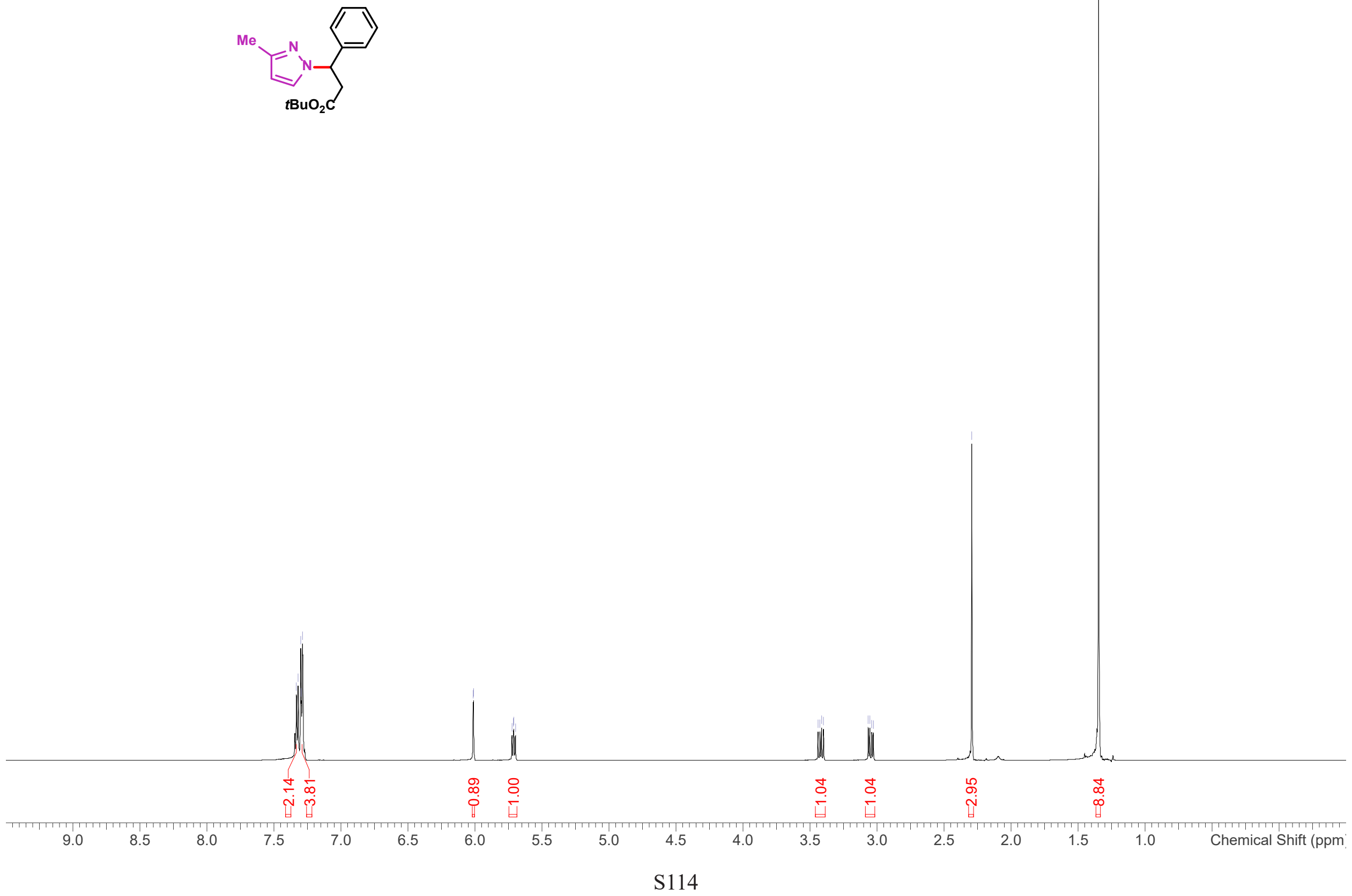
${ }^{13} \mathrm{C} \mathrm{NMR}\left\{{ }^{1} \mathrm{H}\right\}\left(151 \mathrm{MHz}, \mathrm{CDCl}_{3}\right)$ of $\mathbf{8 f}$

怘

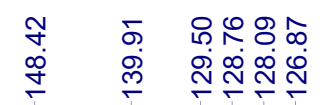

$\stackrel{\infty}{\stackrel{\infty}{\circ}}$

$\stackrel{\infty}{\grave{\infty}}$

ธิ

$\stackrel{\infty}{\stackrel{+}{+}}$

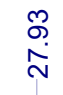

$\stackrel{\infty}{\stackrel{\infty}{\stackrel{m}{m}}}$<smiles>Cc1ccn(C(CC(=O)OCc2ccccc2)c2ccccc2)n1</smiles> 
${ }^{1} \mathrm{H}$ NMR $\left(600 \mathrm{MHz}, \mathrm{CDCl}_{3}\right)$ of SI-13

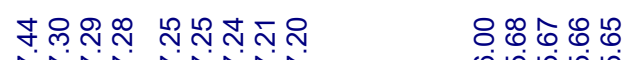

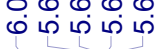

율ํำ

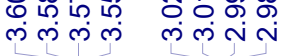

$\stackrel{\Re}{N}$

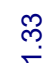
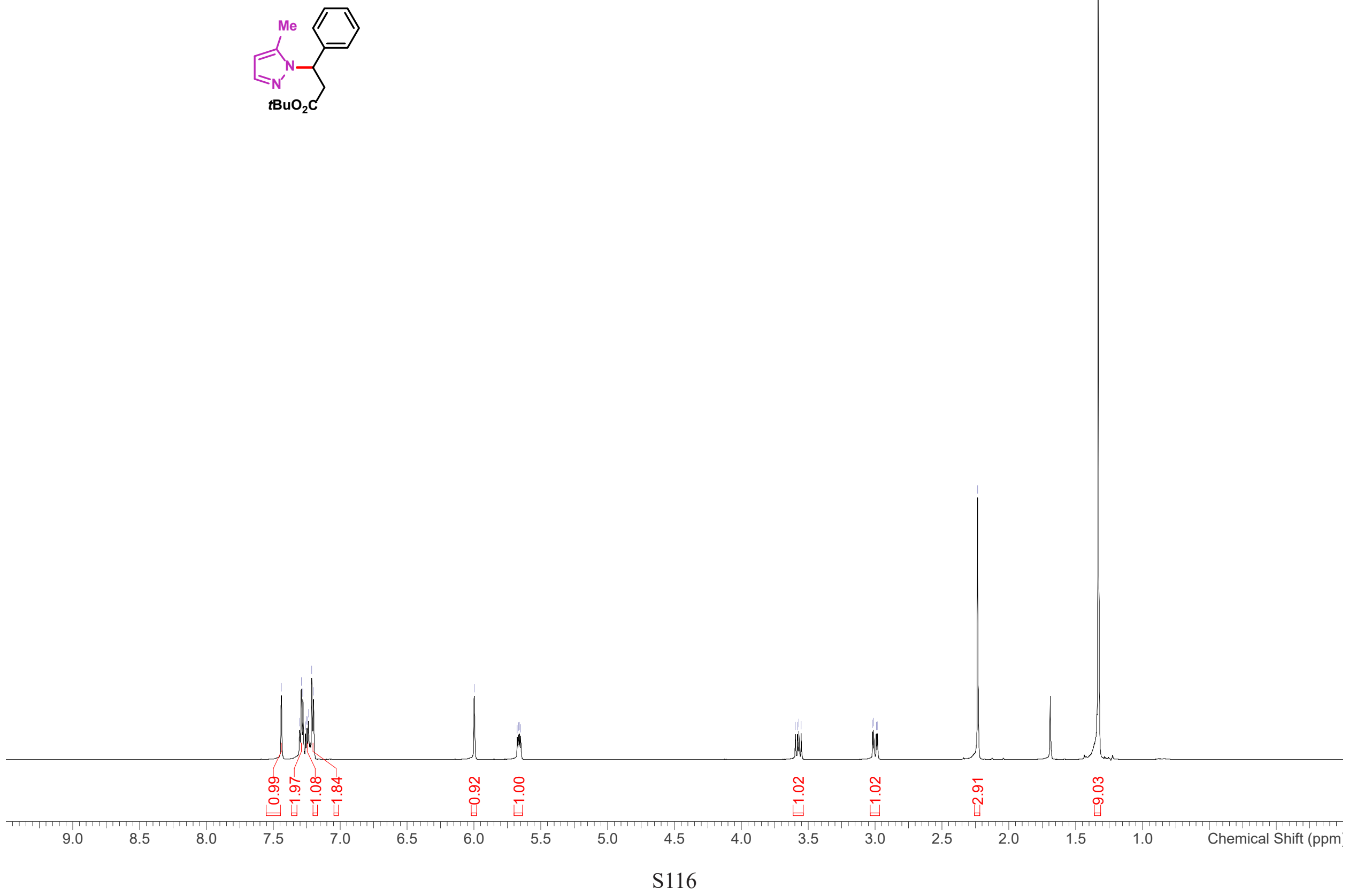
${ }^{13} \mathrm{C}\left\{{ }^{1} \mathrm{H}\right\}$ NMR $\left(151 \mathrm{MHz}, \mathrm{CDCl}_{3}\right)$ of $\mathbf{S I - 1 3}$

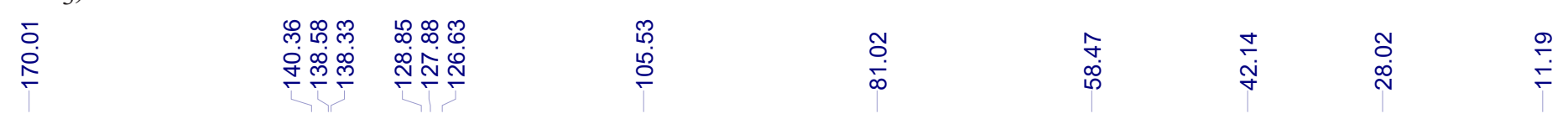<smiles>Cc1ccnn1C(CC(=O)O)c1ccccc1</smiles> 
${ }^{1} \mathrm{H} \mathrm{NMR}\left(500 \mathrm{MHz}, \mathrm{CDCl}_{3}\right)$ of $\mathbf{8 g}$

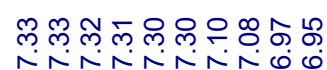

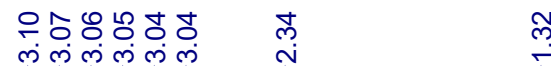

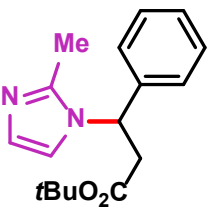

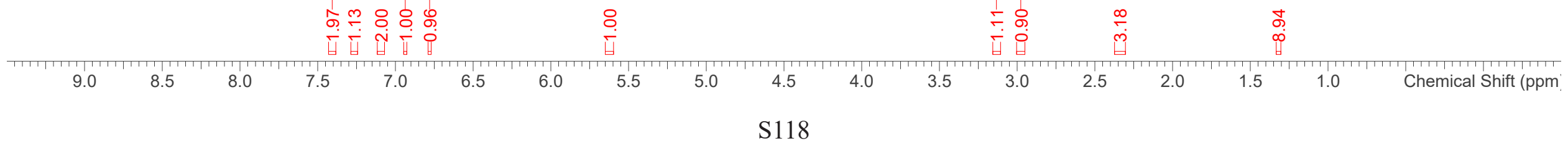


${ }^{13} \mathrm{C}\left\{{ }^{1} \mathrm{H}\right\} \mathrm{NMR}\left(126 \mathrm{MHz}, \mathrm{CDCl}_{3}\right)$ of $\mathbf{8 g}$

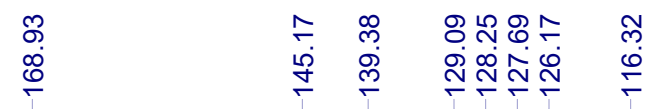

$\frac{\mathrm{\infty}}{\infty}$

กิ.

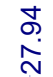

$\stackrel{\leftrightarrow}{\stackrel{0}{p}}$

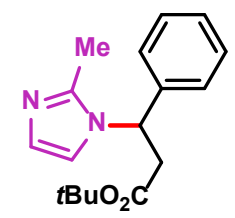

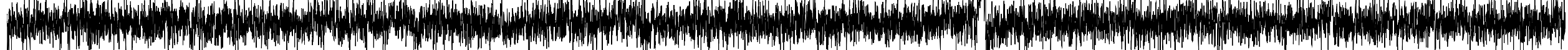


${ }^{1} \mathrm{H} \mathrm{NMR}\left(500 \mathrm{MHz}, \mathrm{CDCl}_{3}\right)$ of $\mathbf{8 h}$

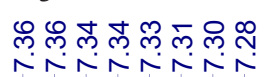

ษัซำㅇำ

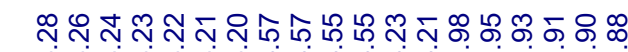

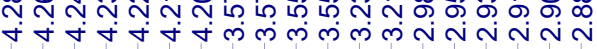

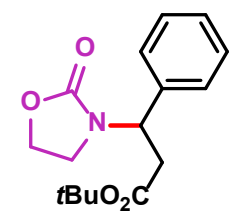

\begin{tabular}{|c|c|c|c|c|c|c|c|c|c|c|c|c|c|c|c|c|c|}
\hline & & & & & & & 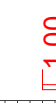 & & & & $\begin{array}{l}\overline{\dot{\zeta}} \\
\dot{\bar{U}}\end{array}$ & 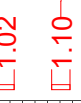 & & & & & \\
\hline 9.0 & 8.5 & 8.0 & 7.5 & 7.0 & 6.5 & 6.0 & 5.5 & 5.0 & 4.5 & 4.0 & 3.5 & 3.0 & 2.5 & 2.0 & 1.5 & 1.0 & Chemical Shift (ppm \\
\hline
\end{tabular}


${ }^{13} \mathrm{C}\left\{{ }^{1} \mathrm{H}\right\}$ NMR $\left(126 \mathrm{MHz}, \mathrm{CDCl}_{3}\right)$ of $\mathbf{8 h}$

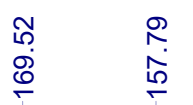

छั. Бे

$\stackrel{\infty}{\infty}$

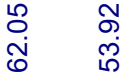

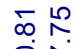

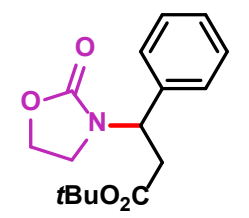

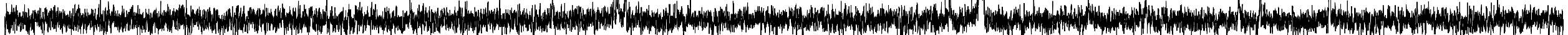

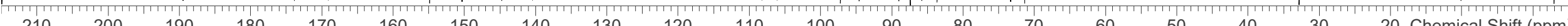


${ }^{1} \mathrm{H} \mathrm{NMR}\left(600 \mathrm{MHz}, \mathrm{CDCl}_{3}\right)$ of $\mathbf{8 i}$

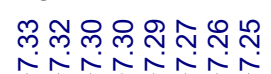
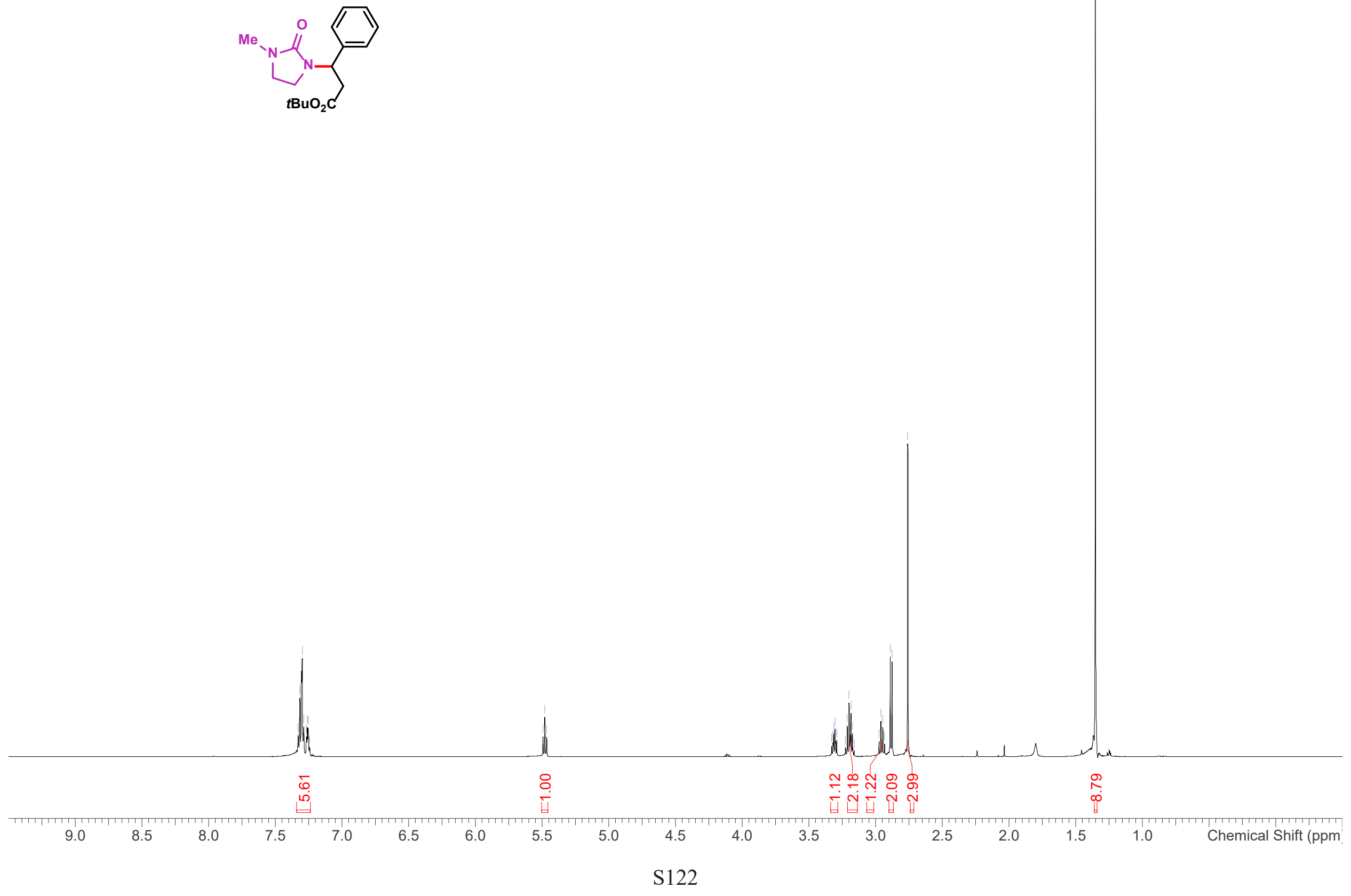
${ }^{13} \mathrm{C}\left\{{ }^{1} \mathrm{H}\right\} \mathrm{NMR}\left(151 \mathrm{MHz}, \mathrm{CDCl}_{3}\right)$ of $\mathbf{8 i}$
$\begin{array}{ll}\stackrel{0}{\circ} & \stackrel{\circ}{\circ} \\ \stackrel{0}{\circ}\end{array}$

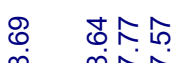
$\frac{8}{\infty}$

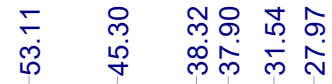
$\stackrel{\infty}{\stackrel{\infty}{ }} \quad \stackrel{\infty}{\sim} N \underline{N}$

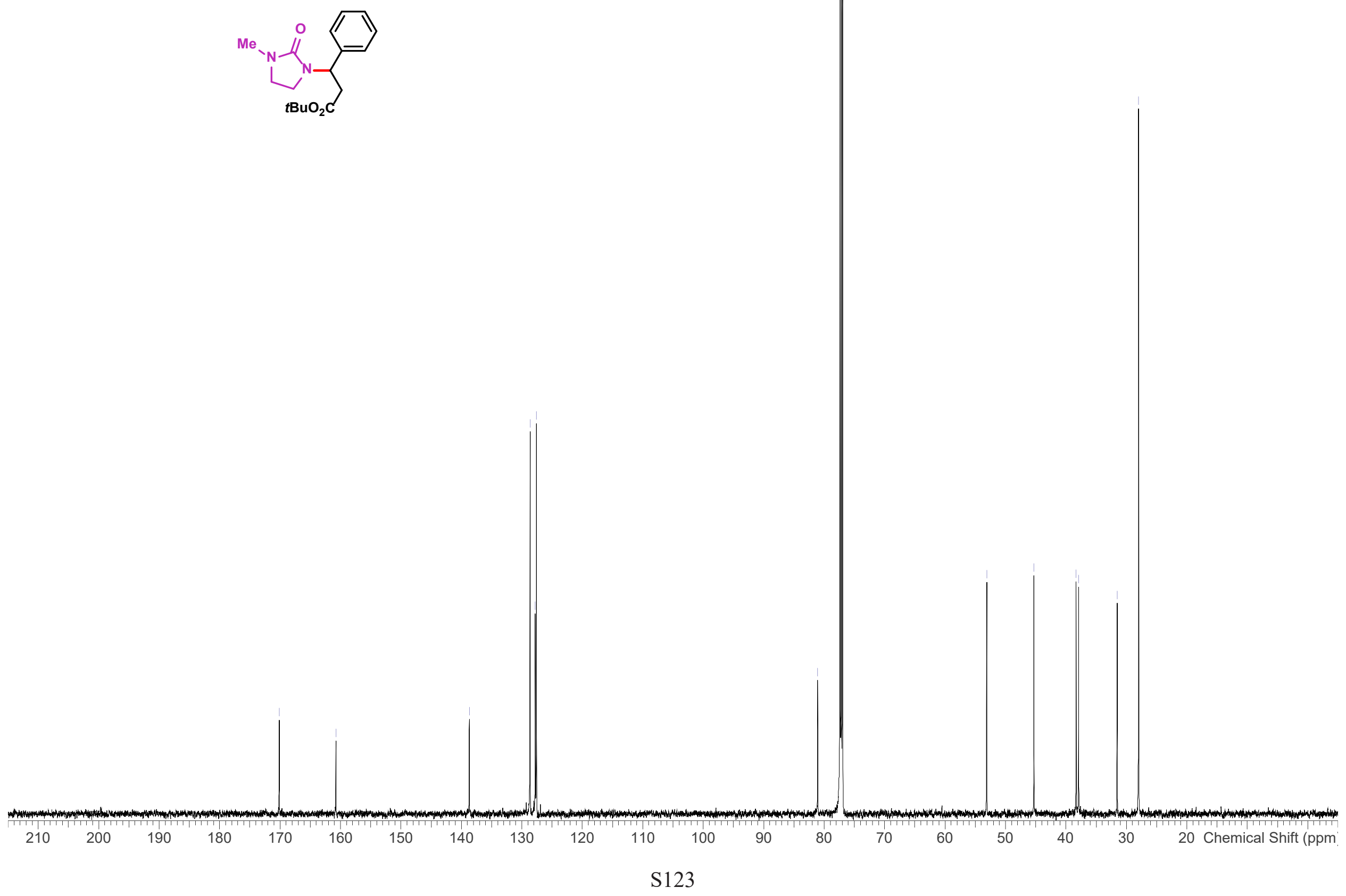


${ }^{1} \mathrm{H} \mathrm{NMR}\left(600 \mathrm{MHz}, \mathrm{CDCl}_{3}\right)$ of $\mathbf{8 j}$

另总

$\sqrt{\infty} \infty \bar{\infty}$

ம்

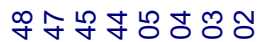
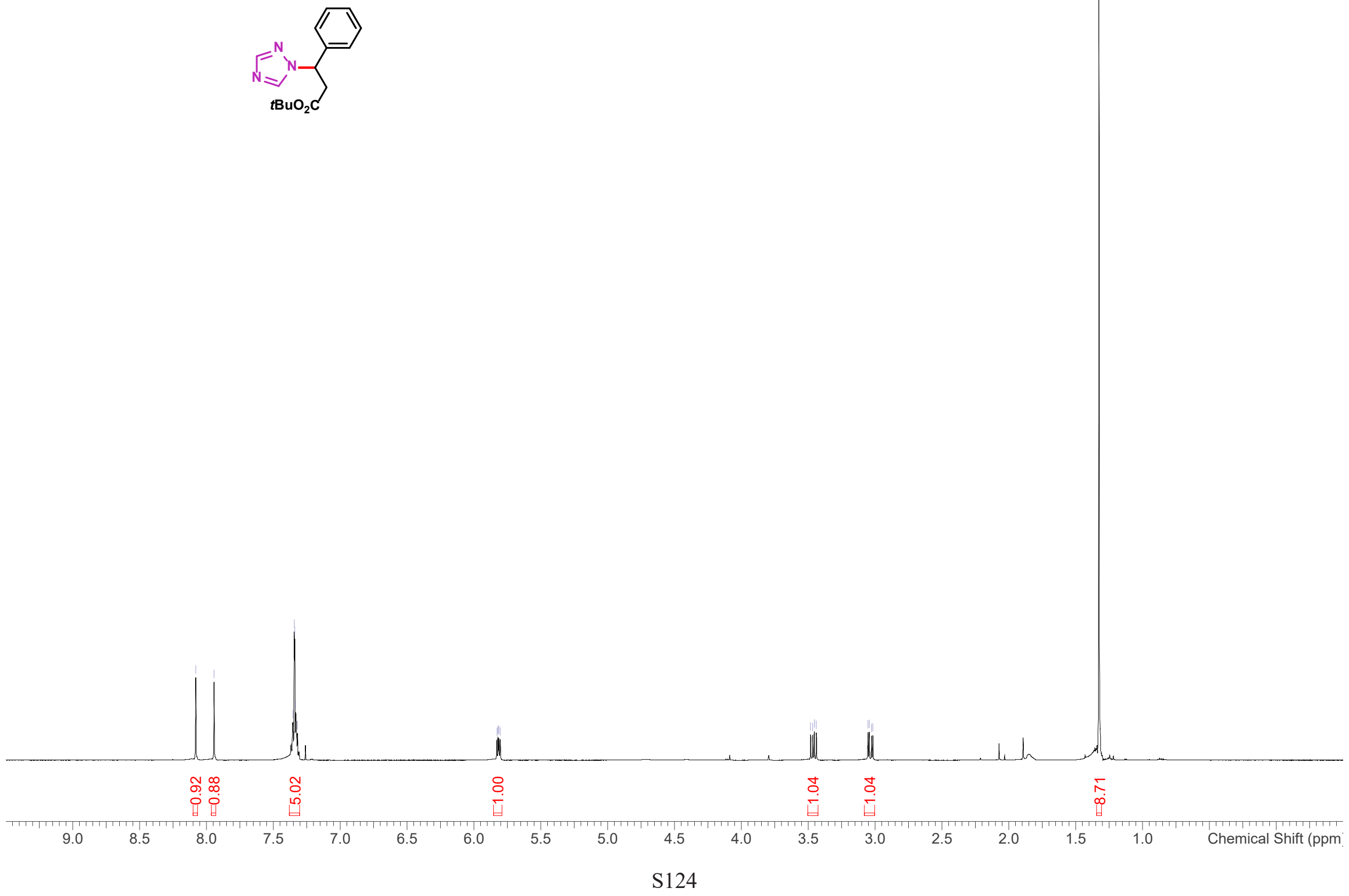
${ }^{13} \mathrm{C} \mathrm{NMR}\left\{{ }^{1} \mathrm{H}\right\}\left(151 \mathrm{MHz}, \mathrm{CDCl}_{3}\right)$ of $\mathbf{8 j}$

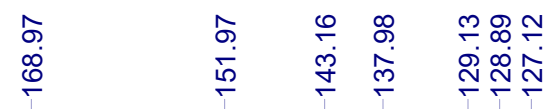

$\stackrel{\infty}{\infty}$

in

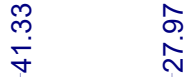

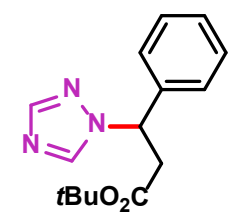


${ }^{1} \mathrm{H} \mathrm{NMR}\left(600 \mathrm{MHz}, \mathrm{CDCl}_{3}\right)$ of $\mathbf{8 k}$

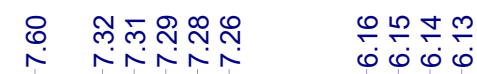

ํํㅇํำ

लه

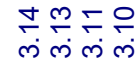

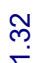

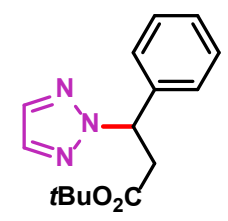

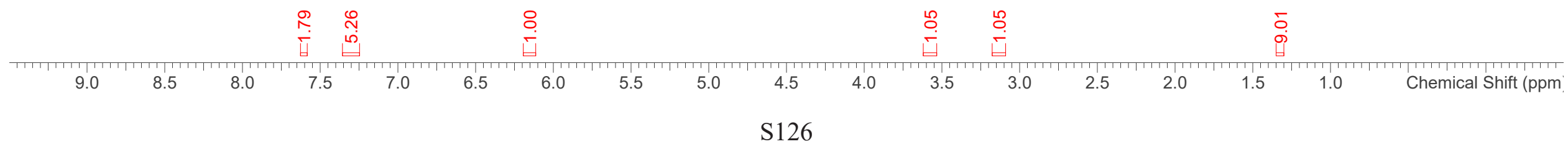


${ }^{13} \mathrm{C} \mathrm{NMR}\left\{{ }^{1} \mathrm{H}\right\}\left(151 \mathrm{MHz}, \mathrm{CDCl}_{3}\right)$ of $\mathbf{8 k}$

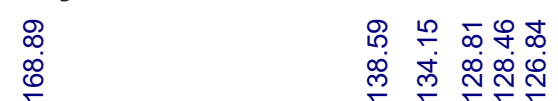

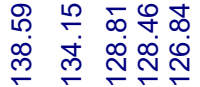

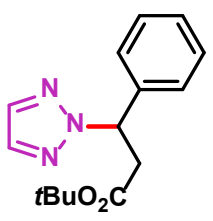


${ }^{1} \mathrm{H}$ NMR $\left(600 \mathrm{MHz}, \mathrm{CDCl}_{3}\right)$ of SI-14

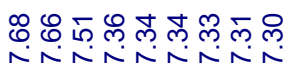

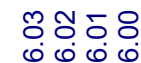

४⿻ำ

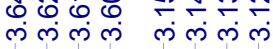
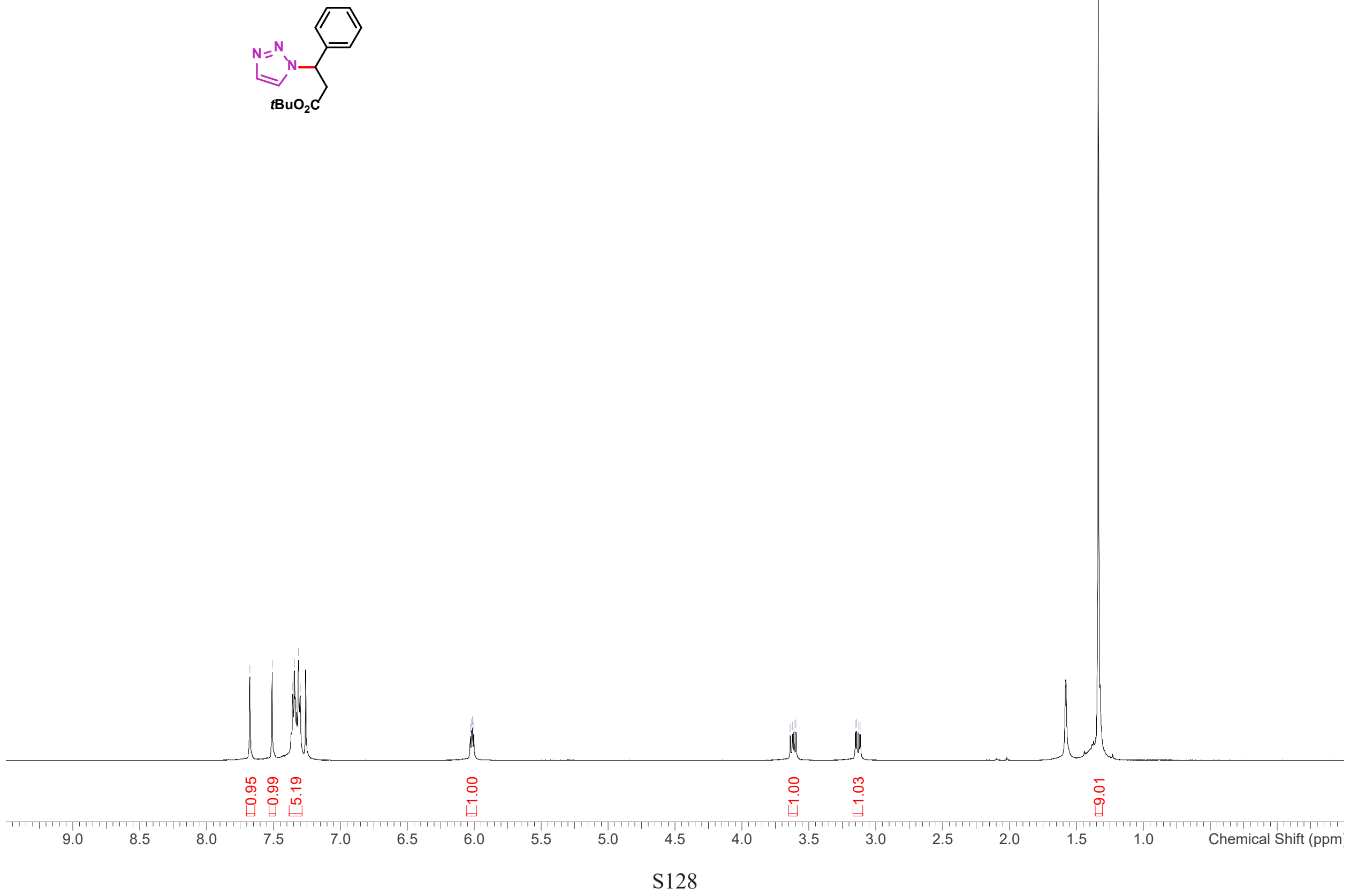
${ }^{13} \mathrm{C} \mathrm{NMR}\left\{{ }^{1} \mathrm{H}\right\}\left(151 \mathrm{MHz}, \mathrm{CDCl}_{3}\right)$ of $\mathbf{S I - 1 4}$

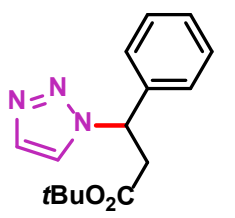




\section{${ }^{1} \mathrm{H}$ NMR $\left(600 \mathrm{MHz}, \mathrm{CDCl}_{3}\right)$ of $\mathbf{8} \mathbf{1}$}

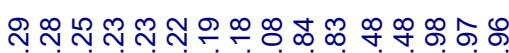

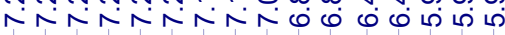

$\mathrm{MeO}$

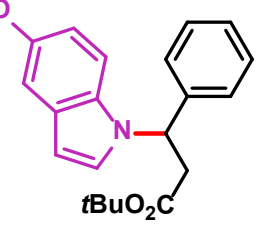

\begin{tabular}{|c|c|c|c|c|c|c|c|c|c|c|c|c|c|c|c|c|c|}
\hline & & & & 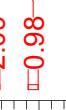 & $\begin{array}{l}\text { J } \\
\text { Oे }\end{array}$ & $\frac{8}{\dot{\leftarrow}}$ & & & & & & & & & & & \\
\hline 9.0 & 8.5 & 8.0 & 7.5 & 7.0 & 6.5 & 6.0 & 5.5 & 5.0 & 4.5 & 4.0 & 3.5 & 3.0 & 2.5 & 2.0 & 1.5 & 1.0 & Chemical Shift (ppm \\
\hline
\end{tabular}


${ }^{13} \mathrm{C}\left\{{ }^{1} \mathrm{H}\right\}$ NMR $\left(151 \mathrm{MHz}, \mathrm{CDCl}_{3}\right)$ of $\mathbf{8} \mathbf{I}$

寈

సิ

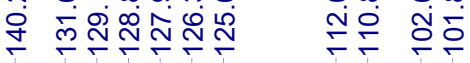

$\mathrm{MeO}$<smiles>O=C(O)CC(c1ccccc1)n1ccc2cc(Br)ccc21</smiles> 
${ }^{1} \mathrm{H} \mathrm{NMR}\left(500 \mathrm{MHz}, \mathrm{CDCl}_{3}\right)$ of $\mathbf{S I - 1 5}$

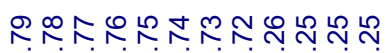

ம்

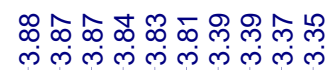

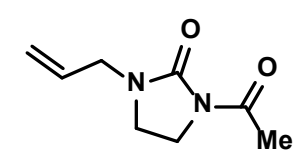

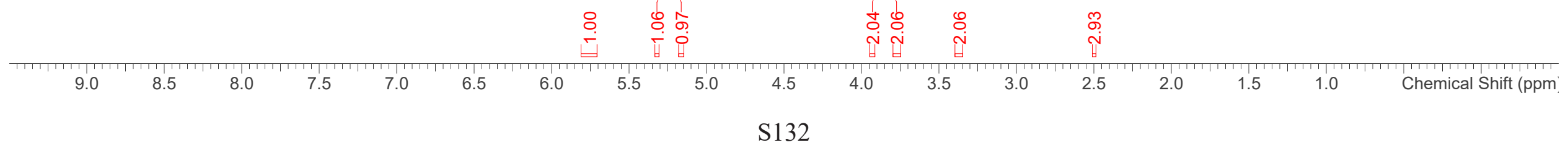


${ }^{13} \mathrm{C}\left\{{ }^{1} \mathrm{H}\right\}$ NMR $\left(126 \mathrm{MHz}, \mathrm{CDCl}_{3}\right)$ of $\mathbf{S I - 1 5}$

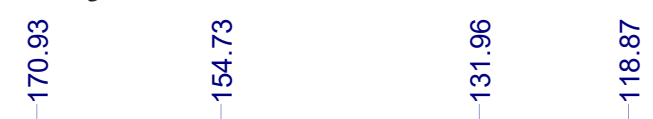

(ำ

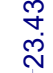

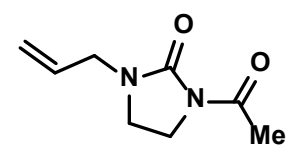

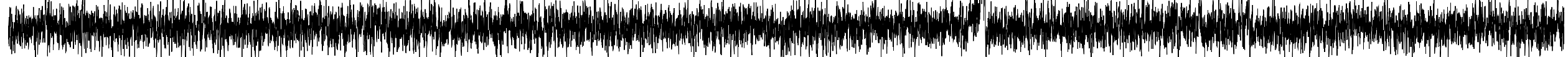

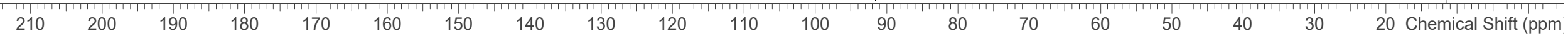


${ }^{1} \mathrm{H} \mathrm{NMR}\left(600 \mathrm{MHz}, \mathrm{CDCl}_{3}\right)$ of $\mathbf{1 0}$

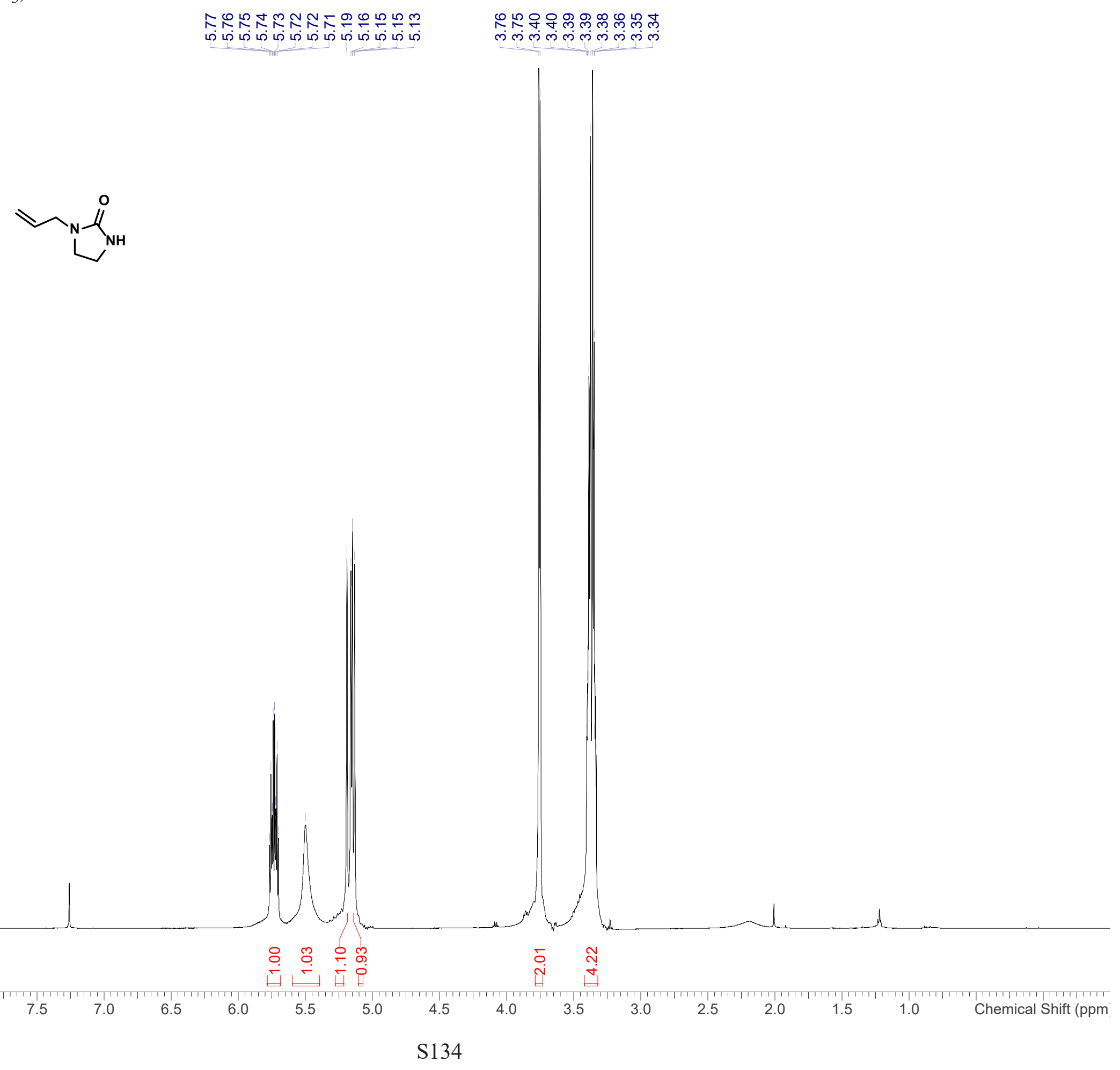


${ }^{13} \mathrm{C}\left\{{ }^{1} \mathrm{H}\right\} \mathrm{NMR}\left(151 \mathrm{MHz}, \mathrm{CDCl}_{3}\right)$ of $\mathbf{1 0}$

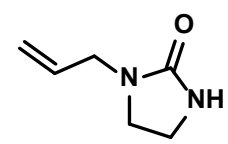


${ }^{1} \mathrm{H} \mathrm{NMR}\left(600 \mathrm{MHz}, \mathrm{CDCl}_{3}\right)$ of $\mathbf{1 1}$

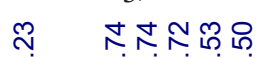

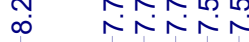

Nㅐ N

00 Oे
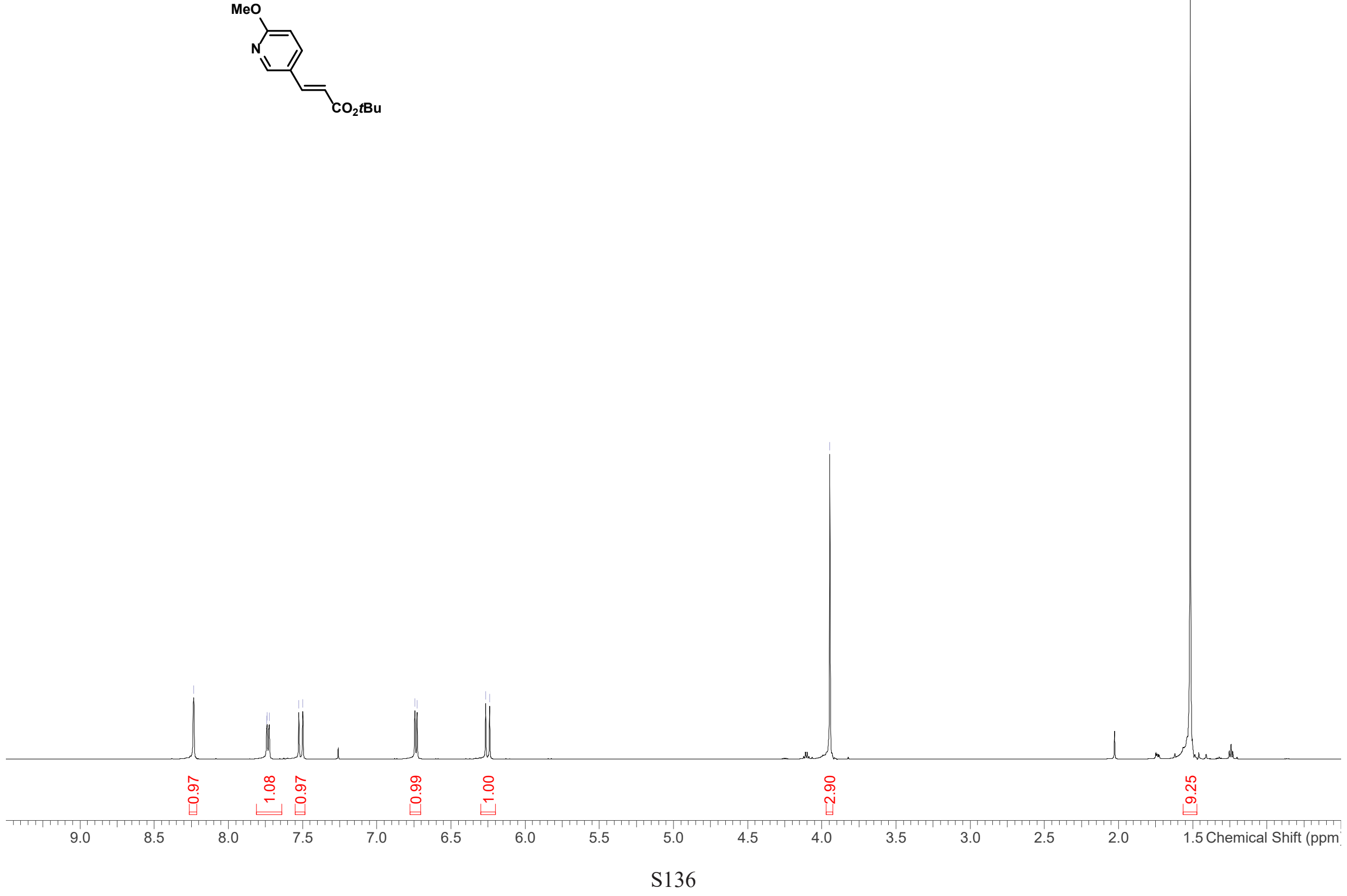
${ }^{13} \mathrm{C}\left\{{ }^{1} \mathrm{H}\right\} \mathrm{NMR}\left(126 \mathrm{MHz}, \mathrm{CDCl}_{3}\right)$ of $\mathbf{1 1}$

六

응

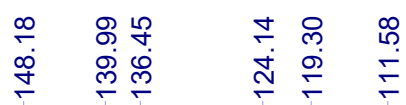

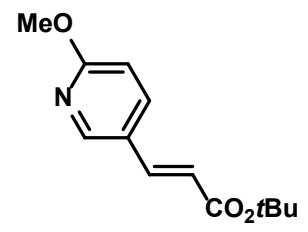

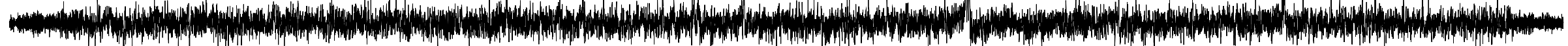

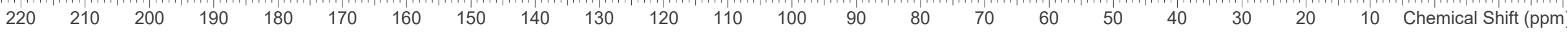


${ }^{1} \mathrm{H}$ NMR $\left(500 \mathrm{MHz}, \mathrm{CDCl}_{3}\right)$ of 12

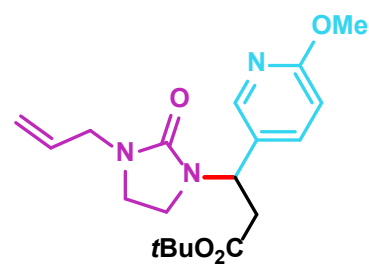

\begin{tabular}{|c|c|c|c|c|c|c|c|c|c|c|c|c|c|c|c|c|c|}
\hline & & $\begin{array}{l}\& \\
\dot{\dot{H}}\end{array}$ & ஜ̊ & & & & gृ & 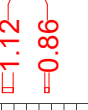 & & 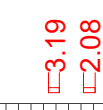 & & 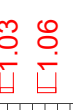 & & & 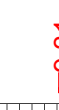 & & \\
\hline 9.0 & 8.5 & 8.0 & 7.5 & 7.0 & 6.5 & 6.0 & 5.5 & 5.0 & 4.5 & 4.0 & 3.5 & 3.0 & 2.5 & 2.0 & 1.5 & 1.0 & Chemical Shift (ppm \\
\hline
\end{tabular}


${ }^{13} \mathrm{C}\left\{{ }^{1} \mathrm{H}\right\} \mathrm{NMR}\left(126 \mathrm{MHz}, \mathrm{CDCl}_{3}\right)$ of $\mathbf{1 1}$

is $\overline{0} \bar{\infty}$

ஜ

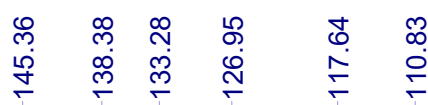

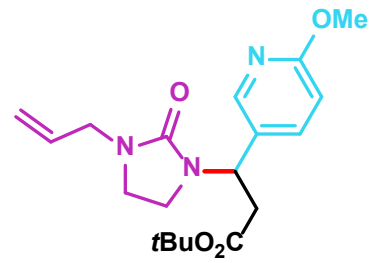


${ }^{1} \mathrm{H} \mathrm{NMR}\left(600 \mathrm{MHz}, \mathrm{CDCl}_{3}\right)$ of $\mathbf{1 4}$

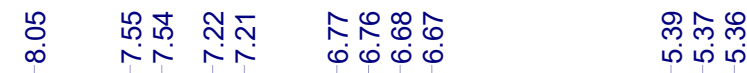

舟

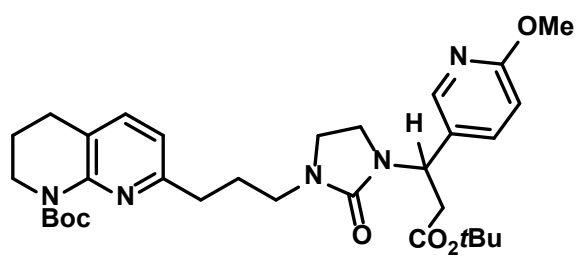

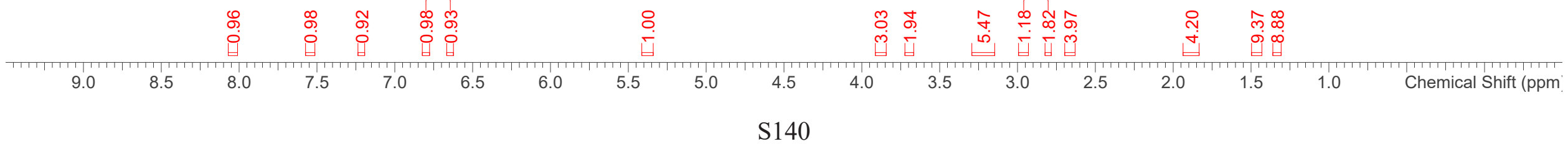


${ }^{13} \mathrm{C}\left\{{ }^{1} \mathrm{H}\right\} \mathrm{NMR}\left(151 \mathrm{MHz}, \mathrm{CDCl}_{3}\right)$ of $\mathbf{1 4}$

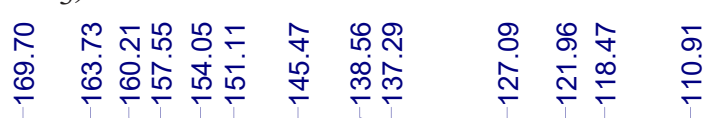

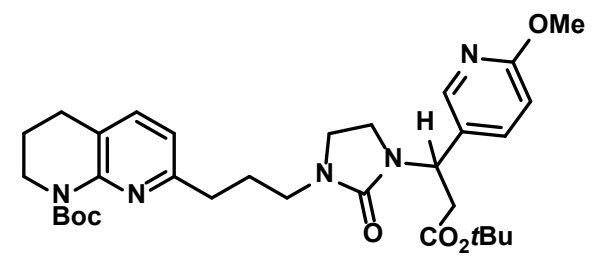


${ }^{1} \mathrm{H}$ NMR (500 MHz, $\left.\mathrm{CD}_{3} \mathrm{OD}\right)$ of 1

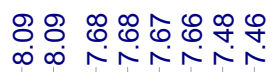

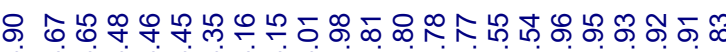
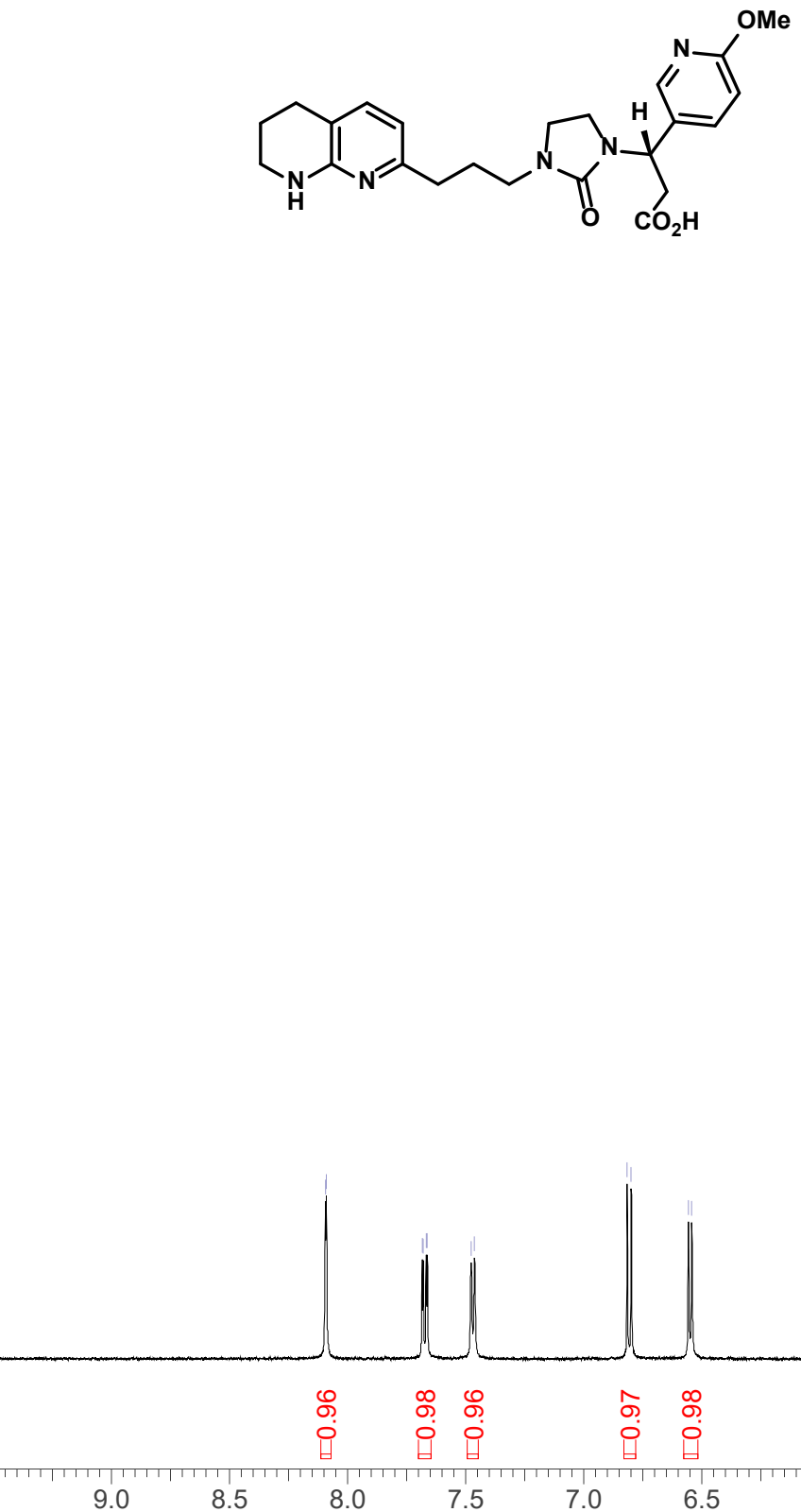

\section{ลิ ๙

草

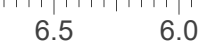

6.0

5.0 
${ }^{13} \mathrm{C}\left\{{ }^{1} \mathrm{H}\right\} \mathrm{NMR}\left(126 \mathrm{MHz}, \mathrm{CD}_{3} \mathrm{OD}\right)$ of $\mathbf{1}$

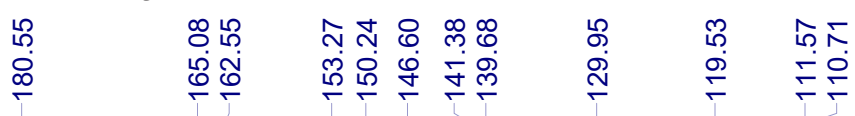

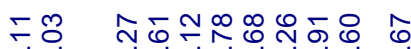

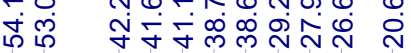<smiles>COc1ccc(C(CO)N2CCN(CCCc3ccc4c(n3)NCCC4)C2=O)cn1</smiles> 